LPNHE 98-XX

\title{
Reconstruction and Particle Identification for a DIRC System
}

\author{
M. Benayoun ${ }^{1}$, L. Del Buono, Ph. Leruste \\ ${ }^{1}$ LPNHE des Universités Paris VI et VII, IN2P3-CNRS, Paris, France
}

November 9, 2018

\begin{abstract}
We study the reconstruction and particle identification (PID) problem for Ring Imaging devices providing a good knowledge of the direction of the Cerenkov photons, as the DIRC system, on which we specialize. We advocate first the use of the stereographic projection as a tool allowing a suitable representation of the photon data, as it allows to represent the Cerenkov cone always as a circle. We set up an algorithm able to perform reliably a fit of circle arcs of small angular opening, by minimising a true $\chi^{2}$ expression. The system we develop for PID relies on this algorithm and on a procedure able to remove background photons with a high efficiency. We thus show that, even when the background is large, it is possible to perform an efficient PID by means of a fit algorithm which finally provides all the circle parameters ; these are connected with the charged track direction and its Cerenkov angle. It is shown that background effects can be dealt without spoiling significantly the reconstruction probability distributions.
\end{abstract}

Keywords : data analysis methods, event shape analysis, particle identification. Free Keywords : Rich detector reconstruction, DIRC reconstruction. 


\section{Introduction}

Cerenkov Ring Imaging devices settle specific problems of pattern recognition. Generally, devices are designed in such a way that one has to look for photons projected onto a circle [1, 2], the radius of which being simply connected to the Cerenkov angle of the charged track radiating these photons. In RICH detectors (as for DELPHI), the main problem is due to background photons which come together with the real signal photons and render difficult pattern recognition, basically because the number of independent constraints which can be used is small. Traditionally, current methods in pattern recognition follow more or less the ideas from Baillon [3], assume the knowledge of the charged track information and perform a maximum likelihood fit to the number of background and/or signal photons, under the various mass assumptions.

It is not of current knowledge to attempt a full fit of the ring parameters, mainly because background photons are expected to spoil the fit quality, and anyway prevent to define a reasonable $\chi^{2}$ to be minimised and then a fit probability having the properties expected for a probability distribution. This is mainly due to the difficulties encountered when trying to select a set of photons associated with a track and possibly still affected by contamination from background photons.

Motivated by the DIRC device [4, 5], which will be used by the BaBar Collaboration [6] at the SLAC PEP-II B-factory, we reexamine this problem with the goal of associating to a given charged track, a set of photons with a low level of contamination, having in mind to be able to check in each photon sample the effect of a residual background contamination. This provides several problems to be solved in order to have a procedure able to run under realistic conditions.

In a DIRC device, one can define with a controlled accuracy the Cerenkov photon directions. This allows to reconsider the problem of data representation, with the aim of having a well defined geometrical figure to look for. We advocate here that the stereographic projection provides such a suitable tool. Whatever are the errors, it allows to look always for an image of the Cerenkov ring which is surely a circle arc, without any special hardware request on the DIRC device considered. This is true even if the charged track direction is poorly known, possibly even unknown. In real life however, because of background, one cannot completely avoid some knowledge - even approximate and/or subject to systematic errors - of informations about the charged track direction. In this representation, the radius of this circle is connected in a simple way with the Cerenkov angle, and the circle center with the charged track direction. The stereographic projection has also been proposed as a tool for pattern recognition円 with a $\mathrm{RICH}$ detector which will use a 27 Kton water target and radiator in order to detect neutrino oscillation at Gran Sasso, using a long base line neutrino beam from CERN [7].

In working conditions, one is faced with another problem, specific to the DIRC : generally, the part of the Cerenkov cone populated by observed photons represents a relatively small azimuthal window compared with $360^{\circ}$. In any representation of the data, this corresponds to a small circle arc populated with measured points. Taking into account the relative magnitude of the errors affecting these points and the circle radius, this generally prevents a direct use of standard circle fit algorithms. Indeed, in such conditions, the fit quality is poor, even in

\footnotetext{
${ }^{1}$ We thank T. Ypsilantis (INFN, Bologna) for this information and for having drawn our attention on the corresponding references.
} 
absence of background, as the radius is systematically underestimated. In order to circumvent this difficulty, a further information has to be used and it happens that the charged track direction is a good candidate for this purpose. This is basically the idea of Ref. [3], but in a completely different context. Indeed, one can always assume that we have some knowledge about the charged track direction provided by some tracking device located in front of the DIRC. However, this implies a deep change in the circle fit algorithm structure. The pattern recognition problem of the long base line RICH of Ref. [7], even if not trivial, is much simpler than for the DIRC as the circle arc to reconstruct is generally quite large and the errors affecting photon directions are much smaller.

In this way, one is led to introduce as measurements to be fit the angular distances between each of the various photon directions and the charged track direction. This introduces strong correlations which have to be carefully accounted for when constructing the expression for the $\chi^{2}$ to be minimised. These correlations are partly due to the measurement errors on the charged track direction and partly due to multiple scattering effects undergone by the charged track inside the radiator ; this last kind of correlations can be important in identifying tracks of very low momentum (typically below $1 \mathrm{GeV} / \mathrm{c}$ ) and they survive even if the circle arc populated by photons is large enough that we need not worry about circle center information.

Finally, having defined appropriate tools for pattern recognition and particle identification, it remains to define a procedure able to provide a set of photons really associated with the track. As the number of constraints available for photon recognition is small, tools allowing to control background contamination effects in this sample are needed.

The paper is organised as follows. In Section 2, we briefly outline the DIRC structure and properties relying on known literature [4, 5], in its aspects relevant to reconstruction and particle identification. In Section 3, we recall the properties of the stereographic projection and the connection with Cerenkov cone reconstruction. Section 4 is devoted to describing the circle fit algorithm and recognition procedure when the circle arc populated by measured points is small ; we also sketch here how to deal with photon contamination control and background removal. In section 5, we describe the Monte Carlo we have coded in order to check the full procedure ; all effects affecting the recognition procedure are included and varied from minimum (to check the basic model properties) to a maximum (photons produced by several tracks in the same bar, with or without additional flat background). The procedure of photon selection and background removal is described in details in section 6, it is more specific to the DIRC problem than the fit itself. This procedure relies on using first a clean subsample of photons (unambiguous photons) to start an iterative recognition procedure. In section 7 we describe, the working of the procedure and show that it behaves well, even under very large background conditions ; the effects of correlations are specifically illustrated and it is shown that they cannot be neglected. Of course, photon selection relies on cutting out photons candidates ; then, Section 8 illustrates how cut levels can be adjusted and tuned in such a way that the probability distributions are not too much affected. We thus show that pull distributions and probability distributions allow to perform the background removal with a controlled quality.

Finally, two appendices contain a full treatment of the multiple scattering effects and correlations. We describe here also our modelling, where all effects are taken into account at first order only. Monte Carlo results show that the effects of this approximation are small in the 
region where a specific particle identification is expected to be reliable with a DIRC device, i.e. above $500 \mathrm{MeV} / \mathrm{c}$.

\section{Outline of a DIRC Device and Properties}

The DIRC (Detection of Internally Reflected Cerenkov light) is a new type of detector which will be used as the main particle identification system in the BaBar experiment [6] at PEP2 collider (SLAC). A prototype of the DIRC [5] has been extensively tested in the CERN PS beam in 1995/1996, demonstrating the validity of the DIRC concept. It will not be described here in full details. We will rather briefly outline the general principles involved in its conception and operation, more information can be found in recent literature on the BaBar DIRC (see Refs. [5], [4] and [8]). We mainly limit ourselves to the aspects of relevance for the procedure we develop and the Monte Carlo we will use in order to test it.

The originality of a DIRC device is that, contrarily to most other Cerenkov ring imaging detectors, it makes use of the Cerenkov light generated in the radiator medium by trapping photons (through total internal reflection) into the radiator itself and guiding them toward a set of photomultipliers (PMTs) for detection ; this also allows the detector to be quite thin in the direction of the incoming tracks, because the Cerenkov cone expands outside the main sensitive area of the detector.

In the DIRC, the radiator is made of long rectilinear fused silica bars of modest rectangular section, a material chosen mainly because of its high refractive index (1.474) [5, 8] compared to air or nitrogen and its long absorption length in the UV region. As sketched in Fig. 1, the quartz/air interfaces of the bars act as perfect mirrors for a wide range of Cerenkov photons incidence angles, and, for a sufficient optical and geometrical quality, they are able to transport the photons to the bar exit, with unperturbed directions except for reflection symmetries with respect to the bar faces.

The number of photons produced in the quartz bars depends on several parameters. Interestingly, it tends to increase with the incidence angle (since the path length inside the radiator medium grows), which is a behavior rather opposite to more traditional ring imaging detectors, where a large part of the Cerenkov light is lost because of the internal reflection inside the radiator.

To avoid loosing too much light at the bar exit, the array of photomultiplier windows and the light quartz bar exits are immersed in pure water, which refractive index (1.34) is close to the quartz one (1.474), so that photons crossing the quartz/water interface have a low probability of being reflected back into the bar.

There is also a reflecting device parallel to the bottom bar surface put at the bar end (in the water) in order to redirect toward the PMTs the photons emerging from the bar with either downward or too upward going directions ; the other bar end is equipped with a small mirror in optical contact with the quartz for the same purpose.

Once the Cerenkov image is detected on the PMTs array, two informations are available : the location of the hit PMT and the photon detection time. This image (see Fig. 2 for an example from the DIRC prototype tested at CERN [5]) is in fact a superposition of different 
reflections of the original Cerenkov cone by the bar reflecting surfaces. So, a given hit can belong to the Cerenkov cone centered on the original track direction, or to any of its images with respect to the reflecting surfaces of the bar. In the case of the prototype of Ref. [5, 8 reflected photon directions (due to 3 possible symmetry reflections planes) are to be taken into account. This ambiguity problem is specific to the DIRC : given a definite photon direction, there are as many track-photon associations as reflection planes and it may be that several combinations are physically acceptable. Fortunately, it may also happen that only one solution is acceptable ; this defines unambiguous solutions. Fig. 目 also illustrates that the arc populated by the hits can be relatively small (of the order of 60 degrees).

Using the spatial information, one is able to reconstruct uniquely the original Cerenkov angle of a photon (which is emitted on the Cerenkov cone) if the ambiguities related with the various possible reflections that could affect the photon during its propagation can be disentangled. The timing information is mainly used for a preliminary recognition of background photons, generally out of time for a given track, as the information provided by the individual photon detection time is very poor compared to the spatial information given by the PMTs.

Thus, a possible strategy for pattern recognition in the DIRC is effectively to try discriminating spatially between photon ambiguities in order to determine the correct symmetry assignment, and then use the set of non ambiguous resulting photon Cerenkov angles (from the whole image) in order to compute the relevant quantities we are interested in : the track Cerenkov angle and its error. This is achieved through a fitting procedure, and these informations allow in a second step to refine the choice among the ambiguous photon solutions left provisionally aside, and take part of them into account in a final fit. Among the difficulties that are to be met, the smallness of the arc length populated by photons should be noticed and has to be especially addressed.

In the following sections, we detail the different parts of the algorithm implemented in order to achieve this goal.

\section{Pattern Recognition Using the Stereographic Projec- tion}

In a transparent medium of index $n$, Cerenkov photons are emitted by a charged particle with an angle $\theta_{C}$ with respect to the charged track direction and this angle is given by :

$$
\cos \theta_{C}=\frac{1}{n \beta}
$$

where $\beta$ is the speed of the particle. As the photon wavelength [/ is generally not measured, this turns out practically to assume that the refractive index $n$ is known with a random error $\delta n$, independently for each photon.

Let us associate to each of the charged track and photon directions a unit vector, and draw all of them from a common origin denoted $(0,0,0)$. All end points of these vectors lay on a

\footnotetext{
${ }^{2}$ We postpone to Appendix A commenting on the influence of chromaticity fluctuations (dependence of $n$ on the photon wavelength $\lambda$ ) which affects each photon direction.
} 
unit sphere, and all photon directions generate a cone of half aperture $\theta_{C}$ around the charged track direction. From now on we shall always refer to photon and charged track directions only in this representation.

\subsection{The Stereographic Projection}

The intersections of the photon directions with a plane perpendicular to the charged track direction at unit distance from the origin define a set of points distributed along a circle of radius $\tan \theta_{C}$, centered at the intersection of the charged track direction with this plane. However, if the actual charged track direction is not the one chosen in order to define the plane, the figure represented by the intersections of the photon directions with this plane is no longer a circle but an ellipse, and departure from a circle may become large if the charged track direction is poorly known 91, because of systematic errors in the measurement of the charged track direction, or misalignments effects.

A way to circumvent this problem (or minimize it at least) is to use the stereographic projection[ [10]. Let us briefly recall it. Let us choose on the unit sphere defined above the pole axis along the measured charged track direction. The stereographic projection of points on the sphere is the intersection of the lines joining the south pole $(0,0,-1)$ to these points with the equatorial plane. In this transform, a circle drawn on the sphere (i.e. the intersection of the Cerenkov cone with the sphere) is projected out as a circle. Then the Cerenkov cone centered along the charged track direction becomes a circle of radius $\tan \theta_{C} / 2$, centered at the origin. This origin is simply the image of the charged track direction, i.e. the projection of the north pole as seen from the south pole. This is sketched in Fig. 3. Ref. [7 prefers performing the stereographic projection onto a plane tangent to the sphere at the north pole, rather than onto the equatorial plane ; correspondingly, the algebra is slightly modified with respect to what will be presented just below.

In practical applications, however, we only know approximately the direction of the charged track, and therefore the pole axis as defined above coincides only approximately with the actual charged track direction. In order to illustrate what happens, let us assume that the actual unknown charged track direction makes an angle $\alpha$, possibly large, with the pole axis (i.e. the reconstructed charged track direction). Then, by means of the stereographic projection, the images of the photon directions in the equatorial plane are still on a circle (see Fig. 3), the radius $R$ of which being :

$$
R=\tan \frac{\theta_{C}}{2} \frac{1+\tan ^{2} \frac{\alpha}{2}}{1-\tan ^{2} \frac{\theta_{C}}{2} \tan ^{2} \frac{\alpha}{2}}
$$

and the circle center is shifted from the origin to a point located at distance $r_{0}$ :

$$
r_{0}=\tan \frac{\alpha}{2} \frac{1+\tan ^{2} \frac{\theta_{C}}{2}}{1-\tan ^{2} \frac{\theta_{C}}{2} \tan ^{2} \frac{\alpha}{2}}
$$

\footnotetext{
${ }^{3}$ Basically, it is a standard conformal mapping of the sphere onto a plane, i.e. angles on the sphere are conserved in the projection.
} 
close to the image of the actual charged track direction, which is located at $\tan \alpha / 2$.

It is clear from rels. (2) and (3) that the error on the circle center is of first order in $\alpha$, while the corresponding error on the radius is only of second order. Therefore, the robustness of the stereographic projection follows from the fact that the analytical shape of the Cerenkov figure in the equatorial plane is always a circle, even if the actual charged track direction is quite different from the measured one. Moreover, the radius is affected at second order only by angular errors on the charged track direction. If the angle $\alpha$ were large, it is clear from Rels. (2) and (3) that having determined by fit $R$ and $r_{0}$ allows anyway to reconstruct the correct Cerenkov angle. Stated otherwise, the pole axis used in order to perform the stereographic projection may be chosen independently of any assumption on the charged track direction. In general, we have :

$$
\tan \theta_{C}=\frac{2 R}{1-R^{2}+r_{0}^{2}} \simeq \frac{2 R}{1-R^{2}}\left[1-\frac{r_{0}^{2}}{1-R^{2}}\right]+\mathcal{O}\left(r_{0}^{4}\right)
$$

If $r_{0}$ is small enough, $R$ is negligibly affectedf but can easily be corrected.

The correspondence between the coordinates of a point on the unit sphere $(X, Y, Z)$, and those of its image $(x, y)$ into the equatorial plane (through the stereographic projection) is defined by :

$$
x=\frac{X}{1+Z} \quad, \quad y=\frac{Y}{1+Z} \quad, \quad\left(X^{2}+Y^{2}+Z^{2}=1\right)
$$

This transform is never singular in our case as we have always $Z>0$.

\subsection{Handling Measurements and Errors}

Let us assume that the above coordinates $X, Y$ and $Z$ (or any other quantity) are measurable quantities with known covariance error matrix, originating from random distributions $\hat{X}, \hat{Y}$ and $\hat{Z}$; assuming the measurement process unbiased, we can write $\hat{X}=X_{\text {true }}+\delta X$, where $X_{\text {true }}$ is the true (unknown) value of $X$ and $\delta X$ a random variable of standard deviation $\sigma_{X}$ and of zero meanf :

$$
\left\{\begin{array}{l}
<\delta X>=0 \\
<[\delta X]^{2}>=\sigma_{X}^{2}
\end{array}\right.
$$

Correspondingly, for any other measurable quantity, we define a centered error function carrying the corresponding standard deviation $\left(\sigma_{Y}\right.$ or $\sigma_{Z}$, for instance). As we assume the measurement process unbiased, we should have indeed $\langle\hat{X}\rangle=X_{\text {true }}$. Using this language, true and measured values are quantities which differ by first order terms $\mathcal{O}(\delta X)$.

The error functions affecting the coordinates $x$ and $y$ (in the equatorial plane) can be derived by differentiating Eqs. (5) :

\footnotetext{
${ }^{4}$ If $\theta_{C}=500 \mathrm{mr}$, in order that $2 \arctan R$ provides an overestimate of at least $0.1 \mathrm{mr}$, the error on the charged track direction should be, at least, $\alpha \simeq 30 \mathrm{mr}$.

${ }^{5}$ We shall always use the notation $\langle f\rangle$ for the expectation value of any random variable $f$.
} 


$$
\left\{\begin{array}{l}
\delta x=\frac{1}{1+Z} \delta X-\frac{X}{(1+Z)^{2}} \delta Z \\
\delta y=\frac{1}{1+Z} \delta Y-\frac{Y}{(1+Z)^{2}} \delta Z
\end{array}\right.
$$

When estimating errors using these expressions, $X, Y$ and $Z$ should be the corresponding true values. As they are unknown, one classically uses instead the measured central values. In terms of differentials, this lack of information affects second order terms (like $\left.(\delta X)^{2}\right)$. So, at first order, it is legimate to use directly measured values while estimating coefficient functions. It is clear that going beyond first order development in analytic expressions would raise a problem here, as the additional terms would be competing with the (uncontrolled) terms introduced by using the measured values instead of the true ones.

If the measurement $(X, Y, Z)$ is unbiased, the point $(x, y)$ is unbiased too at leading order (i.e. $\langle\delta x>=<\delta y>=0)$. The covariance terms $\left(<[\delta x]^{2}>,<[\delta y]^{2}>\right.$ and $\left.<\delta x \delta y>\right)$ can be computed in terms of $X, Y$ and $Z$ and of their errors and correlations ; when computing them, one has to take into account that $X^{2}+Y^{2}+Z^{2}=1$ and that their error functions are not independent : $X \delta X+Y \delta Y+Z \delta Z=0$.

\section{A Circle Fit Algorithm}

As explained in section 3, using the stereographic projection, the directions of the Cerenkov photons emitted by a charged track are represented by points in a plane laying on a circle. Up to second order terms, the circle center is nothing but the projection of the charged track direction onto the (equatorial) plane of the sphere. Therefore, the reconstruction problem of the Cerenkov angle is replaced by a problem of circle recognition in a plane.

It is a long standing problem to find the most suitable way to perform a circle fit to a given set of points affected by measurement errors (see refs. [11, 12, 13 for instance). The main problems addressed in (necessarily) approximate procedures are :

- linearisation of circle parametrisation

- non-gaussian character of the errors on the circle center coordinates and radius.

In addition to the above mentioned questions, we address two more issues, connected with the BaBar DIRC, namely :

- in any representation, the measured points are not spread out onto the whole circle, but along a relatively small arc (about $60^{\circ}$ degrees). Taking into account the relative magnitude of the error on the points and of the circle radius value, this happens to affect deeply the circle fit quality, if no additional information on the circle center is accounted for.

- there exist correlations among photons as a consequence of the multiple scattering undergone by the emitting charged track. Accounting for further constraints (charged track direction measurement) may introduce further correlations (see Sections 2 and 3 in Appendix $\mathrm{B}$ for instance). 


\subsection{The $\chi^{2}$ for Fitting a Circle Arc}

Let us assume that we have $n_{\gamma}$ measured points $\left(x_{i}, y_{i}\right)$ with random errors $\left(\delta x_{i}, \delta y_{i}\right)$, not necessarily gaussian. As we restrict our study to a DIRC device where the points are actually photons, we shall use indifferently the words photon and point. We do not state any assumption on these errors, except that the measurements are unbiased :

$$
<\delta x_{i}>=<\delta y_{j}>=0 \quad, \quad \forall i, j=1, \cdots n_{\gamma}
$$

Stated otherwise, the expectation values $\left.<\delta x_{i} \delta x_{j}\right\rangle,\left\langle\delta x_{i} \delta y_{j}>\right.$ and $<\delta y_{i} \delta y_{j}>$ which define errors and correlations are not constrained and no further assumption is needed on higher order moments. In the approach we have followed, the effects of multiple scattering are not affected to the photons measurements but rather to the track direction.

We assume that we have, beside the circle points, also a measurement of the circle center coordinates and its error; we define our reference frame in such a way that this measured center is located at the origin. The circle parameters we fit are $a$ and $b$ (the center coordinates) and $R$ (its radius). This parametrisation is examined in full details in Appendix B.

The $\chi^{2}$ to minimise in order to get the best circle fitting $n_{\gamma}$ points is defined by (see Appendix B 4) :

$$
\chi^{2}=\sum_{i, j}\left(d_{i}-R\right)\left(d_{j}-R\right) V_{i j}^{-1}+A^{t} \Sigma^{-1} A
$$

with :

$$
d_{i}=\sqrt{\left(x_{i}-a\right)^{2}+\left(y_{i}-b\right)^{2}}
$$

where $a$ and $b$ are the circle center coordinates parameters (measured as $(0,0)$, up to errors) to be fit. We have denoted by $A$ the vector $(a, b)$ of the center coordinate and by $A^{t}$ its transposed. The error matrix $\Sigma$ depends on the reconstruction errors of the track at the DIRC entrance $\left(\Sigma_{0}\right)$, on the number of photons which take part to the fit $\left(n_{\gamma}\right)$ and on the multiple scattering undergone by the charged track inside the radiator ; it is explicitly computed in Appendix A and in Sections 2 and 3 of Appendix B.

In usual approaches this second contribution to the $\chi^{2}$ is not considered [11, 12, 13] ; however, when the circle center is constrained by an auxiliary measurement, it is legitimate to use it. Moreover, it is harmless to remove it only if the circle length populated by the measured points is large enough (typically greater than $180^{\circ}$ ) and/or if $\sqrt{\left\langle\left[\delta d_{i}\right]^{2}>\right.} / d_{i}$ is small enough. In the case of the DIRC, none of these conditions are practically met, and removing the constraint on the center represented by the second term in the RHS of Rel. (9) may simply lead to a complete failure of the circle fit, even in absence of fake photons.

The matrix $V$ which appears in Rel. (9) is the error covariance matrix. It is also the matrix of the error function expectation values :

\footnotetext{
${ }^{6}$ Actually, the vector $(a, b)$ should be written $\left(a-a_{\text {measured }}, b-b_{\text {measured }}\right)$; however we take into account that the measurement has been conventionally set at $(0,0)$.
} 


$$
V_{i j}=<\delta d_{i} \delta d_{j}>\quad, \quad \forall i, j=1, \cdots n_{\gamma}
$$

The error function $\delta d_{i}$ affecting the measurement $i$ is given by :

$$
\delta d_{i}=\frac{x_{i}\left(\delta x_{i}+\delta a_{i}\right)+y_{i}\left(\delta y_{i}+\delta b_{i}\right)}{d_{i}}
$$

(see Appendix B4) up to higher order terms. In this expression, we use as central values for the circle center coordinates the point $(0,0)$, while the error functions on the circle center are estimated for each photon. It is the reason why the error functions which appear in Rel. (12) are $\delta a_{i}$ and $\delta b_{i}$, referring to the charged track direction when it emits photon $i$ and $\delta x_{i}$ and $\delta y_{i}$ are the measurement errors of the direction of photon $i$. The form of the error functions for $\delta a_{i}$ and $\delta b_{i}$ is given in Appendix B1, using preliminary results from Appendix A. The form of the error function $\delta d_{i}$ is explained in Appendix B4; the elements of the matrix $V$ in Rel. (11) are computed in Section B 5. One can see there how correlations terms like $\left\langle\delta a_{i} \delta a_{j}>\right.$, $<\delta a_{i} \delta b_{j}>$, ... produce non zero correlation terms in $\left\langle\delta d_{i} \delta d_{j}>\right.$. Consequently, one can interpret $a$ and $b$ in Rel.(10) as the mean values (to be fit) of the sets $\left\{a_{i}\right\}$ and $\left\{b_{i}\right\}$. Actually, the single information - except for errors - we have on these sets are the measured values at the bar entrance ; how this is accounted for is explained in details in Appendix B.

\subsection{Linearisation of the Circle Parameter Equation}

It remains to linearise Eq. (9). The procedure is quite usual [11, 12, 13 and turns out to replace Eq. (9) by :

$$
\chi^{2}=\sum_{i, j}\left(d_{i}^{2}-R^{2}\right)\left(d_{j}^{2}-R^{2}\right) \frac{V_{i j}^{-1}}{4 R_{0}^{2}}+A^{t} \Sigma^{-1} A
$$

where $R_{0}$ is an estimate of the radius (a weighted mean of the $d_{i}$ at start and, in the forthcoming steps of the iteration the fit value of $R$ at the previous step of the iteration). The matrix $V$ depends on the circle center coordinates. They are chosen here at start as $(0,0)$, and can also be

updated in the forthcoming steps of the iteration procedure. The final step toward linearisation is to define as fitting parameter $c=R^{2}-a^{2}-b^{2}$ instead of $R$, together with $a$ and $b$. Then Eq. (13) becomes :

$$
\left\{\begin{array}{l}
\chi^{2}=\sum_{i, j} \mathcal{C}_{i} \mathcal{C}_{j} W_{i j}^{-1}+A^{t} \Sigma^{-1} A \\
W_{i j}=4 R_{0}^{2} V_{i j} \\
\mathcal{C}_{i}=x_{i}^{2}+y_{i}^{2}-2 a x_{i}-2 b y_{i}-c
\end{array}\right.
$$

and this last expression for $\chi^{2}$ is quadratic in $a, b$ and $c$. Eqs. (13) and (14) simply follow from the fact that near the minimum we have :

$$
\left(d_{i}-R\right)=\frac{\left(d_{i}^{2}-R^{2}\right)}{\left(d_{i}+R\right)} \simeq \frac{\left(d_{i}^{2}-R^{2}\right)}{2 R_{0}}
$$


This relation fastly improves when iterating, and few iterations only are needed in order to be at less than $10^{-2}$ from $\chi_{\min }^{2}$ (generally 2 steps are enough for the accuracy just quoted).

The conditions defining the minimum are :

$$
\frac{\partial \chi^{2}}{\partial a}=0 \quad, \quad \frac{\partial \chi^{2}}{\partial b}=0 \quad, \quad \frac{\partial \chi^{2}}{\partial c}=0
$$

and provide a linear system of equations for $a, b$ and $c$ which gives an optimum solution to the minimisation problem. On the other hand, denoting the variables $a, b$ and $c$ by $u_{\alpha},(\alpha=1,2,3)$, Eqs. (14) can be written (summation over repeated indices is understood) :

$$
\chi^{2}=T_{\alpha \beta} u_{\alpha} u_{\beta}+Z_{\alpha} u_{\alpha}+K
$$

where the matrix $T$, the vector $Z$ and the scalar $K$ can easily be expressed in terms of the (given) $W$ and $\Sigma$ matrices and of the photon coordinates $\left(\left[x_{i}, y_{i}\right], i=1, \cdots n_{\gamma}\right)$ moments. In addition, the matrix $T^{-1}$ is the error covariance matrix for the fit parameters $\left(u_{\alpha}, \alpha=1,2,3\right)$ [16]. This covariance matrix gives the error contour at $\chi_{\min }^{2}+1$, the $1 \sigma$ contour.

The number of degrees of freedom associated with the $\chi^{2}$ in rel. (14), is $n_{\gamma}-1$ and then the fit probability is the value of the $\chi^{2}$ probability function $\operatorname{Pr}\left(\chi^{2}, n_{\gamma}-1\right)$. One can also define a consistency check of the set of photons under consideration with the set of track parameters : the charged track direction providing the expected coordinates of the circle center $a_{0}=0$ and $b_{0}=0$ (the measured values) and the -five- possible values of the circle radius corresponding

to the -five- possible mass assignments for the charged track, $R_{k}(k=1, \cdots 5)$. In this case, the $\chi^{2}$ simplifies to :

$$
\left\{\begin{array}{l}
\chi^{2}=\sum_{i, j} \mathcal{C}_{i} \mathcal{C}_{j} W_{i j}^{-1} \\
W_{i j}=4 R_{k}^{2} V_{i j} \\
\mathcal{C}_{i}=x_{i}^{2}+y_{i}^{2}-c_{0}
\end{array}\right.
$$

where $c_{0}$ takes five possible values $c_{0}=R_{k}^{2}$, each corresponding to one of the possible values of $R_{k}=\tan \theta_{C}^{k} / 2$. In this way, one can check the consistency of the set of photons considered with the measured charged track parameters $\left(a_{0}=0, b_{0}=0, R_{k}\right)$ for each of the five possible mass assignments. The $\chi^{2}$ just above corresponds exactly to $n_{\gamma}$ degrees of freedom. One can then decide to choose the best assignment as being the one which corresponds to the lowest $\chi^{2}$, provided it is above some significance threshold (a lower probability cut). We illustrate in the following that the corresponding probability distributions have all expected properties.

\subsection{Fit Likelihoods, Fake Photons and Contamination}

Let us denote $\chi_{n_{\gamma}-1}^{2}$ and $\chi_{n_{\gamma}}^{2}$, the $\chi^{2}$ defined resp. by Rels. (14) and (17). Given a set of photons, it is clear that, using standard formulae, the former $\chi^{2}$ allows to define a maximum likelihood including the center measurement, while the latter leads to the likelihoods of the full measured track with the full photon set considered, for each possible mass assignment. 
In practical applications, however, it should be noted that defining the true (maximum) likelihood, implies that :

- the photons considered are indeed photons,

- the photons are actually connected with the track considered,

- the photons errors are correctly estimated.

This sets the problem of background photons. Indeed, in the DIRC, actual photons have errors which are well approximated by gaussians and can be computed, more or less accurately, with known information (geometry, chromatic errors, ...). If an observed hit is not an actual photon (noise) or if it is an actual photon but emitted by another track, possibly from another quartz bar, its error distribution (and its standard deviation) are completely unknown ; therefore, their actual contribution to any $\chi^{2}$ cannot be estimated with any controlled accuracy.

Stated otherwise, any procedure aiming at providing a reasonable estimate of the $\chi^{2}$ probability (or of any likelihood), should remove background photons from the photon sample kept in the fit and the $\chi^{2}$ estimates. On the other hand, it is clear that, in presence of noise, any estimate of the $\chi^{2}$ is altered, except if one would be able to remove background from the photon set at the $100 \%$ level, which is generally hopeless.

Fortunately, the level of actual contamination in any photon set left by any cleaning up procedure can be checked statistically. Indeed, as can be seen from Rels. (13) - (16), the fit solution found for $R$ (denoted here $R_{f i t}$ ) and its error $\sigma_{R}$, crucially depend on the photons used, their errors and their mutual correlations, all informations which can be accessed with a good accuracy. If the photon set is too much contaminated, the fit solution may depart significantly from the expected solution.

The pull $\left(R_{f i t}-R_{\text {true }}\right) / \sigma_{R}$ should follow a centered gaussian law of unit standard deviation. This property provides a quantitative criterium to test the quality of background removal. Indeed, the most likely effect of background is to shift $R_{f i t}$ from its expected value ; this is reflected in the pull distribution by a shift of its mean value from zero and an increase of its rms with respect to 1 . The magnitude of observable departures from the standard pull expectations clearly signals a more or less acceptable level of contamination, as soon as a correct pull behavior has been ascertained with noise-free samples. Therefore, checking the model with noise-free samples is an unavoidable step in constructing the procedure.

When running with Monte Carlo data, all checks can easily be performed ; when running with real data, several checks are still possible using selected events samples like pions from $K_{S}^{0}$ decays or protons from $\Lambda$ decays, which can be selected using kinematics only, i.e. independently of the DIRC. Assuming that background conditions are not especially dependent on the existence of such particles in the events, the quoted pull can be plotted and the influence of background contamination inferred.

\subsection{Pattern Recognition Using a Circle Fit Algorithm}

In practical applications we have a collection of hits that are associated with Cerenkov photons emitted by a given track. These hits are of three different kinds :

- photons emitted by the track under consideration 
- photons emitted by other tracks than that under consideration

- background hits associated with electronic noise of photomultipliers or to unidentified tracks (which practically can be merged with noise).

Hits of the first kind have generally well behaved error functions (not far from gaussians, if not exactly gaussians), while the other two kinds of hits have practically unknown error distributions and should be removed in order to give a statistical meaning to the circle fit. Stated otherwise, the photon sample must be cleaned up. There is also, specific to the DIRC device, another category of fake photons (the ambiguity problem) which will be addressed in Section 6 .

Basically, the cleaning procedure of the photon sample relies on the fact that photons, actually emitted by the track considered, have error distributions which can be well approximated by gaussians. In this case, a suitable criterium in removing fake photons is to eliminate all hits giving a contribution to the $\chi^{2}$ greater than some maximum value.

Therefore, the recognition procedure turns out to perform a fit with a starting sample of photons, in order to have an estimate of the circle parameters $\left(a, b\right.$ and $R$ ), then compute the $\chi^{2}$ distance of each photon to this circle, remove those which are too far, and restart the procedure with the surviving photons ; the procedure is repeated up to convergence. At this point, one can consider that the circle parameters are reliable and reexamine all possible photons in order to keep those which are at acceptable $\chi^{2}$ distance from the expected circle (typically less than about 9). In this way, one recovers ambiguous photons which were put aside in order to start the reconstruction procedure. Using this new enlarged sample, one can then perform a final circle fit and get the optimum circle parameter values. Practically, in the case of the DIRC, there are some subtleties which allow to improve background rejection and photon recovering ; they will be described with more details in Section 6 .

\section{Simulation of a simplified DIRC}

A fast Monte Carlo program was written in order to test the reconstruction algorithm. We have coded this program in order to output all needed information allowing to check the algorithm behavior in full details, which is not usually an easy task within a Monte Carlo simulating a complete experimental detector. This program simulates only one quartz bar and a PMT array plane $(3 \mathrm{~cm}$ diameter PMTs packed in a rectangular lattice, located at approximately $120 \mathrm{~cm}$ from the bar exit). The angle of the PMT plane with respect to the bar axis could be chosen. The bar itself was 5 meters long, which is approximately twice longer than the DIRC prototype bar of Ref. [5], but corresponds to the actual size of the bars of the BaBar DIRC [6]. The distance between the bar exit face and the PMT plane has been chosen following BaBar DIRC setup.

The simulation included most part of effects that could hamper DIRC performances:

- Errors on track direction and momentum, supposed to simulate the response imprecision of a tracker in front of the DIRC bar. In current running conditions we have chosen $\sigma(p) / p=3 \cdot 10^{-3}$ and the generated angular errors have $\mathrm{rms} \sigma(\theta)=\sigma(\phi)=1 \mathrm{mr}$. 
- Realistic detector geometry (geometrical uncertainties are important in the DIRC)

- Chromaticity in the quartz radiator medium (dependence of medium index $n_{Q}$ - and hence Cerenkov angle - on photon wavelength); it corresponds to choosing $\delta n_{Q} / n_{Q}=610^{-3}$.

- The ratio $g$ of the water to quartz refractive indices was treated as independent of the photon wavelength. This is close to the real situation, where $\delta g / g$ is typically $10^{-3}$.

- Track multiple scattering in the quartz bar.

- Number of Cerenkov photons emitted along the track proportional to $\sin ^{2} \theta_{C}$ and to the charged particle path inside the radiator medium. The number of generated photons per centimeter has been chosen [5, 8] as $N_{0}=135$.

- The bar end opposite to the water tank bar exit window is treated as a mirror.

- Full account of photon reflection and refraction properties inside the bar, providing a correct bar acceptance simulation.

- The active part of the PMT plane has been truncated to half a plane, in order to prevent getting fully populated Cerenkov rings.

In this simulation, the geometry and detector structure are incomplete (compared to the final BaBar DIRC [G]): there is no reflecting wedge ending the quartz bar at the water tank entrance (suppressing one reflection plane for photons) and geometrical imperfections of the bar (expected to be very small anyway) are not simulated. The PMT array here is a plane while in BaBar detector, it is umbrella shaped [6]. It is packed as a rectangular lattice (while the BaBar packing is hexagonal, introducing in this way additional correlations among geometrical errors, however of limited magnitude).

In addition, no interaction of the track with the bar is taken into account (except for multiple scattering), in particular no energy loss is implemented. The PMTs spectral response function is also not used in the simulation, except for computing the mean water and quartz refractive indices (and their dispersion). No photon absorption inside the bar is accounted for. Finally, there is no magnetic field effect.

To summarize, there is no conceptually important difference between this simulated setup and that of BaBar and, instead, all relevant features are accounted for.

The main data sample used for this study is a set of 5000 single electron, muon, pion, kaon, and proton tracks having a large bar incidence angle and momentum range $\left(20^{\circ}<\theta_{\text {inc }}<\right.$ $70^{\circ}, 0.5<P<5 \mathrm{GeV}$ ). This sample could be used in several ways : in normal mode, one event contained only a single track ; another mode of operation allowed to superimpose several tracks in one event, for physics background studies ; a last possibility was to add a random and (spatially) uniform noise to the event tracks, for other noise studies. These background conditions can thus be made quite severe compared to normal conditions. The quality checks of the whole procedure have been performed accurately by varying at will the magnitude of all errors, the background kind and level, and the phase space window. 
The momentum range where the algorithm has been fully tested goes down to $500 \mathrm{MeV}$, practically the kaon Cerenkov threshold. At this momentum the angular error due to the multiple scattering for a pion is about $10 \mathrm{mr}$ (for a bar thickness of $1.7 \mathrm{~cm}$ ) this is quite comparable to the angular error due to the PMT window size (about $7 \mathrm{mr} \mathrm{rms}$ ). Going to lower momenta is possible ; however, in this case the angular error due to multiple scattering may become dominant (for a $200 \mathrm{MeV}$ pion, it is about $30 \mathrm{mr}$, far above the PMT geometrical error). In this case the procedure still works, however our first order estimates of the errors might become moderately accurate and higher order terms might become necessary 1 .

\section{Photon Selection and Background Removal}

\subsection{Outline of the Procedure}

The linearized $\chi^{2}$ fit we have described needs, as input, photons with unambiguously defined direction with respect to the track momentum : so, the main step of the photon selection procedure is to lower the number of ambiguities arising in the reconstructed photon direction due to the various reflection symmetries of our problem (bar surfaces, mirrors) ; ambiguities should be selected according to criteria which guarantee the symmetrized photon solutions correspond to possible or probable Cerenkov angles for the current track. Ambiguous photons cannot be used directly by the fit and so they are ignored during the first steps of the procedure (part of them will be recovered by a dedicated algorithm, see below). This ambiguity removal is actually performed in several steps using different criteria (detailed in section 6.2).

The next step of the selection procedure is the removal of the possible background photons contaminating the unambiguous photons population ; an iterative cut procedure involving a median estimator is used for this purpose (section 6.4).

The algorithm requires a minimum number of unambiguous photons in order to go on (typically 3, but this number can be lowered to 2). If there are enough unambiguous photons left, the parameters of interest (Cerenkov angle, coordinates of the center of the Cerenkov circle in the equatorial plane) are fit to this set of remaining unambiguous photons. This preliminary fit is made only in order to have a first approximation of these parameters and of their errors.

A second and analogous fit is applied to a photon population built by adding to the primary set of unambiguous photons, photons which were originally flagged as ambiguous, but which have parameters not too far from the parameters resulting of the primary fit, according to the $\chi^{2}$ distance criterium . These additional unambiguous photons should also fulfill another condition which guarantees they are "unambiguous", at least to some extent : for each new photon, the two ambiguities closest to the central parameters should be themselves sufficiently separated in the relevant $\chi^{2}$ distance (see section 6.5).

This last fitting operation produces a new (and final) determination of the circle fit parameters and errors.

\footnotetext{
${ }^{7}$ Actually, as below $\simeq 700 \mathrm{MeV}$, the main PID device in BaBar is the drift chamber by means of the $\mathrm{dE} / \mathrm{dx}$ measurements 8], it does not seem useful to go to such complications. An overlap region of about 200 to 300 $\mathrm{MeV}$, where an optimum reconstruction can be performed with the DIRC and using dE/dx, allows already interesting cross-checks.
} 


\subsection{Ambiguity Identification and Removal}

\subsubsection{Ambiguous - Unambiguous Cerenkov Photons}

In our problem, each hit recorded in the DIRC PMT array is associated with a reconstructed track. This primary association is made using straightforward criteria : for each hit, the 8 symmetrized Cerenkov angles are selected by requiring that they lay into a physically meaningful interval (typically, bounded by the Cerenkov angles corresponding to the extreme mass hypotheses).

This step is important, because it controls the total number of PMT hits/tracks that will be examined by the full reconstruction algorithm, and hence influences significatively the time performance of the algorithm. After this primary simple association step, one PMT hit/track pair usually still gives rise to several Cerenkov angle solutions. These different solutions are called "ambiguities" hereafter : assigning a unique Cerenkov angle to a photon for one track turns out to discriminate between these ambiguities.

Usually, this primary association step leaves an average number of approximately 2 ambiguities per hit, so that a large part of the photons are still ambiguous. To further select our sample of "solutions", we restrict even more the allowed range of Cerenkov angles around the various mass hypotheses, through a cut in the $|\delta \theta|=\left|\theta_{\text {solution }}-\theta_{\text {hypothesis }}\right|$ variable computed for each solution Cerenkov angle. This cut is made typically at the level of $30 \mathrm{mr}$ which corresponds to

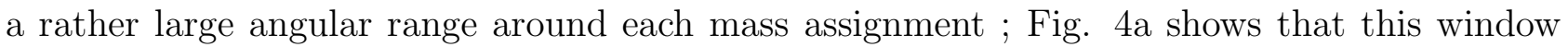
can be naturally defined and checked on a track sample. Solutions found in the allowed range around at least one of the five mass hypotheses are considered. As $\theta_{\text {hypothesis }}$ is computed from the track momentum and a mass assumption, it is always in a valid range $; \theta_{\text {solution }}$ however is computed from measured angles and mean refractive index, then, even for the correct solution, it may be physically meaningless (i.e greater than the maximum Cerenkov angle). Such values have nevertheless to be kept in order to prevent biasing distributions.

Since other studies [6] have shown that the main background in the DIRC are photons generated by other tracks in the same event, another cut is made, on the same grounds, to cope with this "inter track" noise in multitrack events : each PMT hit/track solution surviving this $30 \mathrm{mr}$ cut is tested versus the other measured tracks of the event to check if it can be associated with another track according to the same $|\delta \theta|$ criterium (at the more restrictive level of $10 \mathrm{mr}$ in this case, see Figure $⿴ 囗 十)$. The second $|\delta \theta|$ cut discards only a limited number of photons but an important number of background solutions.

In order to be classified unambiguous, each photon should give one and only one reflection solution relative to the track considered, such that $|\delta \theta|<30 \mathrm{mr}$ and no solution such that $|\delta \theta|<10 \mathrm{mr}$, relative to any other track in the event.

The levels of the two $|\delta \theta|$ cuts (30 and $10 \mathrm{mr}$ ) have been adjusted such as to correspond to a $3 \sigma$ and a $1 \sigma$ cut; this means they have been kept at a quite loose level, leaving about $99 \%$ of the signal visible by the algorithm. Obviously, these criteria can easily be adjusted without a Monte Carlo, as illustrated by Fig. 田.

\subsubsection{Perpendicular Tracks Recovering}

The previous classification of photons as "ambiguous" or "unambiguous" is not well adapted to the case when the track direction is almost perpendicular or parallel to some surface of the 
quartz bar : in this case, trivial ambiguities appear systematically because of the intrinsic symmetries in the bar + track system, i.e. one allowed Cerenkov angle for this PMT hit/track pair will always generate two valid solutions per trivial symmetry plane, since these reflections have in fact (almost) the same Cerenkov angle.

To cure this problem, a narrow cut on the incidence angle of the track with respect to the bar is made which, for this kind of tracks, runs a procedure discarding systematically these trivial additional solutions, keeping randomly one of them. The magnitude of this cut is mainly related to the geometry of the quartz bar + PMTs system, and in particular it is chosen quite small compared to the angular size of one PMT as seen from the water side bar exit. In our case, the PMTs have a diameter of $3 \mathrm{~cm}$ and are distant from approximately $120 \mathrm{~cm}$ from the bar exit : the corresponding angular aperture is around $25 \mathrm{mr}$ (corresponding to about $7 \mathrm{mr}$ rms); the cut is set at $7 \mathrm{mr}$.

This cut is slightly biasing but, as the trivial ambiguity kept is random, this bias is surely limited. Monte Carlo studies do not show any clear signal of bias related with this cut, moreover the proportion of almost perpendicular tracks can be expected small at least for phase space reasons.

After having applied the two $|\delta \theta|$ cuts and the "perpendicular tracks" recovering cut, photons which still allow for several Cerenkov angle solutions are declared "ambiguous", and photons admitting one and only one solution are "unambiguous". The latter ones are used directly in the rest of the procedure. The three cuts already described are clearly independent of the particle kind.

\subsection{Cut on the Number of Unambiguous Photons}

After the preliminary step described above (step "A"), a first cut on the number of unambiguous photons is made: we require the fit to run with at least 2 photons as input. In principle, one should require 3 photons to be sure to keep a non singular $3 \times 3$ matrix in the minimisation procedure ; nevertheless, the constraint put on the circle center, allows to lower safely this limit to 2 input photons. The mean ratio of the number of unambiguous photons to real photon hits (having survived the primary association cuts) at this point of the reconstruction is around $55 \%$; this ratio obviously depends on the cuts one is using.

When the number of unambiguous photons for the current event is lower than 2, in order to avoid loosing systematically the track, the algorithm tries repeating step A using a less restrictive $|\delta \theta|$ cut level which is lowered from 30 to $20 \mathrm{mr}$ in several steps. This part of the algorithm concerns only a few percents of the events, and no significant bias could be traced back to it. The gain in efficiency of the algorithm after this "smoothed" $|\delta \theta|$ cut is around $5 \%$ of events with two tracks.

\subsection{Background Removal using a Median Cut}

In the previous step, we have already performed the removal of some background effects. Indeed, the ambiguous photons represent a kind of combinatorial background, which is identified and left aside provisionally, awaiting improved informations on the Cerenkov circle from the 
set of unambiguous photons. Moreover, the $10 \mathrm{mr}$ cut on $|\delta \theta|$ drops out already most of the contamination produced by photons associated with other reconstructed tracks, which can affect the unambiguous photon set associated with the correct track,

After the construction of the unambiguous photons sample, it is possible to improve the removal of background photons, or, at least, to remove outlier photons. Further background may originate from several sources :

- Photons created by unreconstructed tracks (low energy tracks produced in the detector materials, backsplashes from a nearby calorimeter, ...)

- Improperly accounted for photon solutions (i.e. wrong reflection hypotheses considered as correct unambiguous solutions).

- Background originating from the accelerator, PMT noise, cosmic rays ... is expected lower than the other types of background.

The first type of background is event dependent and may have a complicated structure, whereas the second and third types are rather flat in Cerenkov angle space (at least on a large range around the nominal Cerenkov angle). However, we have found no significant difference in the reconstruction behaviour between them and thus, they are treated likewise. The second kind of background has been found relatively easy to deal with, at the expense of loosing for the fit strongly ambiguous photons (i.e. solutions which are consistent with more than one image of the Cerenkov cone, even with the improved accuracy allowed by the fit estimation of the circle parameters). These strongly ambiguous photons have not been used in the circle fit ; they can nevertheless be counted in order to improve the information on the total number of photons associated with the track.

In any case, it is possible to cut out noise outside a window centered at the median Cerenkov angle (ideally, around the correct Cerenkov angle) of the set of unambiguous photons associated with a given track. The same window will be used afterwards as the area where the fit will be performed.

The median is used here instead of the mean as central location estimator as it is less sensitive to noise and allows a better window setting (the "mean" location estimator is known to be non robust with respect to outlier tails, see for example [14, 15]).

In conditions where the background is low (and/or mostly produced by other reconstructed tracks), the cut to be performed around the median corresponds to a gaussian cut $\mathrm{f}$ of about $3 \sigma$; in much harder background conditions this cut may have to be lowered to about $2 \sigma$, affecting the probability distribution shape only in the low reconstruction probability region.

The median cut procedure is iterated on the unambiguous photon sample till convergence is reached (i.e. until no new photon is removed), which usually happens after 2 or 3 iterations. The ratio of the number of photons, unambiguous at this point of the procedure, to photons having survived the first association step cuts is approximately $50 \%$.

\footnotetext{
${ }^{8} \sigma$ being defined by a theoretical computation of the gaussian errors.
} 
In case of even higher background (like for example when many unreconstructed tracks are present in the event) the median procedure itself behaves poorly, since background photons may accumulate at several locations in Cerenkov angle space, simulating signal for almost all mass hypothesis. In this case, it is meaningless to rely on simple location estimators (like the median): in particular, the preceding procedure has an identification performance which depends on the track momentum when this one is close to the Cerenkov threshold of the various mass hypothesis. In such heavy background conditions, it is better to perform a fit only in restricted Cerenkov angle areas, and not on the full Cerenkov angle range: in this way, the response becomes more uniform. The choice beetween the two selection methods for the fit window depends on the noise level, and can be done automatically.

After the median step, the sample of surviving photons is supposed to be free at least of the influence of unwanted tails which could spoil the subsequent fit. This preliminary fit gives a first estimation of the Cerenkov angle and the circle center in equatorial plane for our sample of filtered unambiguous photons.

A last "cleaning" cut is applied after this first fit in order to remove outlier photons which have individual Cerenkov angles far from the fitted Cerenkov angle ; these photons could still exist and could degrade the results of the rest of the procedure. Typically, a fixed $3 \sigma$ cut is applied for this purpose.

After this last cut, the unambiguous photons represent about $45 \%$ of the actual population of photons associated with the track. The corresponding spectrum is shown in Fig. 57a.

\subsection{Ambiguous Photons Recovering}

It is possible to get more photons in the final fit after recovering part of the photons previously flagged as ambiguous, through a procedure using a $\chi^{2}$ criterium : those among them which have a contribution to the $\chi^{2}$ (estimated at the reconstructed Cerenkov angle and circle center computed by the first fit) which is not too high are declared unambiguous and included into the fitting sample, with the additional condition that their two best solutions are not too close to each other according to $\chi^{2}$ distance.

Practically, this means that the photon solutions are not farther away from the first fit Cerenkov angle than typically $3 \sigma$ and that the closest among the other solutions is beyond, typically, 3.5 or $4 \sigma$.

This "double $\chi^{2 "}$ criterium allows to input in the second fitting step a sample of photons of the order of $65 \%$ of the original sample (under large background conditions) or about $80 \%$ (no additional background). The gain represented by the recovering procedure thus amounts to $50 \%$ to $100 \%$ of the photon number compared to the sample size before the recovering step. The proportion of finally used photons wrt the detected photons associated with a track is plotted in Fig. 5b for the sample with no additional background.

One may imagine to replace this double $\chi^{2}$ criterium by a single one : keep the best solution provided it is below $\simeq 3 \sigma$, whatever is the $\chi^{2}$ of the second ambiguity solution. In conditions where the background is small, this increases significantly the number of photons - then, the accuracy on the reconstructed angle - and the reconstruction quality. In very hard background conditions, however, one has to study carefully to which extent the subsequent gain in photons does not degrade the reconstruction quality. It is not done here. 


\section{$7 \quad$ Monte Carlo Results}

The results presented in this section have been obtained using the set of cuts defined in Section 6. The numerical values of these cuts have been tuned depending on the background conditions affecting each of the Monte Carlo samples. We postpone to Section 8 the discussion on cut handling and tuning. Here we examine the results obtained from analysing these samples, in order to draw conclusions on the fit quality and the various aspects of background removal.

Another important aspect is the algorithm performance for what concerns the separation power between the various mass hypotheses, more directly related to the use of the reconstruction for physics. Many ways to estimate this performance can be devised ; here we will discuss only simple criteria. Indeed, even if background effects are realistic in our Monte Carlo, and sometimes pessimistic, actual performances can be precisely defined only with a detailed simulation of a given experiment or with real data. Moreover, the "performance" requested depends strongly on the physics goals one is willing to achieve.

\subsection{One Track Event Display}

In order to substantiate the problem of pattern recognition and background removal when dealing with the DIRC, it is not useless to display some events. For this purpose, we show in Figs. 6 and 7 an event with one track (actually a kaon of $1.087 \mathrm{GeV} / \mathrm{c}$ momentum) superimposed with a low flat background (additional number of PMT hits at the level of about $20 \%$ of the signal PMT hits). The stereographic projection has been performed with the polar axis along the bar axis and then, the Cerenkov circles are not centered at the origin. Circles corresponding to the original track are drawn thick (the outer one looks even thicker as the $e, \mu$ and $\pi$ assumptions gives circles nearly superimposed), their images with respect to all symmetry planes are the shaded circles shown in only the upper Fig. 6. The lower Fig. 6 displays the same region with only the circles associated with the original track direction under the various mass assumptions. The points represented are the solutions surviving the primary association cut (see Section above) ; the shaded area is the region of zero acceptance.

Fig. 6illustrates the task of the recognition and expresses as clearly as possible the usefulness of the charged track information ; most of the background shown is produced by ambiguities. This means that this event is relatively clean. The algorithm described above extracts the unambiguous photon subsample (see upper Fig. 7) ; in the present case all unambiguous photons are located along the circle corresponding to the kaon assumption. This is the effect of the median cut referred to above ; indeed, by looking at Fig. 6, one clearly sees that unambiguous photons belonging to the proton circle (the innermost one) have been removed by the procedure.

The subset of ambiguous photons is shown in the lower Fig. 0 ; the photon solutions which are to be examined by the recovering procedure belong to all mass assumptions. The extracted ambiguous photons which will be added to the unambiguous photon sample belong only to the kaon assumption circle. 


\subsection{One Track Events with no Background}

We first analyse single track events generated with no external background. This allows to study the algorithm performances and get quality checks under optimum conditions. The track momentum range goes from 0.5 to $5 \mathrm{GeV} / \mathrm{c}$ and the sample contains equal numbers of $e, \mu, \pi$, $K$ and $p$.

No (flat) random noise is superimposed to the event-tracks and no additional track embedded in these events, but this does not mean that they are background free. Indeed, the "combinatorial" background represented by wrongly assigned reflection assumptions to hits pollute the sets of unambiguous photons. Therefore, we can check simultaneously the behaviour of the algorithm and the removal of wrongly assigned photons. In this case, the median cut for background removal can be put at a very loose value $(\simeq 4 \sigma)$ compared to harder background conditions.

Fig. Ba shows that the reconstruction probability is close to be flat : the mean value is 0.52 (expected 0.50), while its rms is 0.277 (expected 0.288), both parameters being close to expectations for a flat distribution. The bias produced by slightly overcutting in order to remove wrongly assigned (true) photons is thus negligible. The reconstruction quality is illustrated in Fig. 8b , where the Cerenkov angle pull is represented; it is very close to a centered gaussian distribution of unit standard deviation. This means that errors are well understood and correctly accounted for, including multiple scattering handling. This also means that the identification is close to optimumg (pions were identified at the level of $95 \%$ and kaon contamination was about $4 \%)$. Finally, Fig. 8 $8 \mathrm{c}$ shows the bias $\left(\theta_{C}^{\text {true }}-\theta_{C}^{\text {fit }}\right)$; the mean bias found is about $0.3 \mathrm{mr}$, and the spread is about $3 \mathrm{mr}$. The number of tracks sent to the procedure was 5000, the fraction which was found with at least 2 unambiguous photons, and thus reconstructed $\mathbb{D}$, was $88 \%$. Therefore the single significant effect of the combinatorial background is to reduce by about $10 \%$ the number of events with at least 2 unambiguous photons. Nevertheless, if reconstruction quality has to be considered, tracks with at most one unambiguous photon (even when lowering the $|\delta \theta|$ cut to $20 \mathrm{mr}$ ) look somehow suspicious.

The reconstructed Cerenkov angle pulls are plotted for a few bins of track momentum in Fig. 9a: the rms of these pulls are quite stable as a function of the track momentum, and are close to 1 . This stability implies that error estimation is correct, even in relatively small track momentum bins : the non gaussian behaviour of the Cerenkov angle distribution, even if not smoothed by an averaging over the track momentum, looks quite limited. There seems to be a systematic bias (at the $30 \%$ level) at low track momentum which decreases with increasing momenta ; this should be attributed to harder multiple scattering effects. Indeed, one should remark in Fig. 9a that, going to higher and higher momenta, allows at the same time to reduce the histogram bias to smaller and smaller values, while the rms become closer and closer to 1 .

Absolute deviations from the simulated Cerenkov angle are also plotted in the same track momentum bins on Figure 9 $\mathrm{b}$, showing the dependence of the errors on the track momentum, as noticed before. These plots also show that the general shape of the errors distributions in each momentum bin is correct, i.e. not far from a true gaussian.

\footnotetext{
${ }^{9}$ Of course, as expected, the $\pi-\mu-e$ separation is poor in the track momentum range explored.

10 Among the $12 \%$ events lost, about $2.2 \%$ are protons below the Cerenkov threshold.
} 
There is an analogous situation when one examines the dependence of the errors and biases of the reconstructed Cerenkov angle with respect to the track incidence angle on the quartz bar : in this case, Fig. 10 shows clearly the existence of a systematic bias for tracks hitting the bar with a high incidence angle ${ }^{\mathbb{T}}$. This seems to corresponds to the already noticed effect with low momentum tracks, i.e. larger multiple scattering effect, but due to longer paths inside the quartz bar. Indeed, in this case, tracks with high incidence angles may suffer stronger changes compared with low incidence angles. The rms dispersion of the pulls looks here also quite stable when the track incidence angle varies, as demonstrated in the same figure.

Instead of fitting the circle parameters on the photon sample extracted from data, one can decide to look for the $\chi^{2}$ probability (with $n_{\gamma}$ degrees of freedom) by fixing completely the parameters to their values inferred from the charged track direction (see Rel. 17). The result of this exercise is shown in Fig. 11 for true pions and kaons. The probability distribution happens to be flat under the right mass assumptions, while it becomes sharply peaked towards 0 , under the wrong mass assumptions. Then, in addition to unaccurately reconstructed tracks, the very low probability bins may be enriched with tracks carrying wrongly assigned mass. It is usual to take this into account by a probability threshold ; in the present case, a cut at about $2 \%$ looks enough.

\subsection{Effect of Correlations}

The previous sample, affected only by the minimum (combinatorial) background, allows to study at various levels the effects of correlations. To be more precise, the data sample is intrinsically affected by correlations ; a method to study their effects is to remove correlations terms in the analysis, i.e. in the algorithm. It was remarked in Appendix B5, that photons close in azimuth are strongly correlated. On the other hand, we know that the circle arc populated by the Cerenkov photons can be small. Then, one can guess that the net effect of correlations should be indeed strong.

A first way to compute the Cerenkov angle and its error, neglecting all correlations, is to set :

$$
R=\sigma_{R}^{2} \sum_{i} \frac{d_{i}}{\sigma_{i}^{2}} \quad, \quad \frac{1}{\sigma_{R}^{2}}=\sum_{i} \frac{1}{\sigma_{i}^{2}}
$$

and knowing $R_{\text {true }}$, one can compute the pull rms. In these expressions, $\sigma_{i}^{2}$ is the squared error of $d_{i}$. This corresponds to fixing the charged track direction to its central value given by the tracking device. In such a computation one actually neglects correlations in estimating $\sigma_{R}$, since otherwise we would have used $1 / \sigma_{R}^{2}=\sum_{i, j} V_{i j}^{-1}$ (see Rels. (11) and (B.6)) and not only the trace of the inverse of $\mathrm{V}$, which becomes diagonal when correlations are neglected. The corresponding pull is always well centered (reflecting the fact that the charged track direction measurement is unbiased) while the pull rms varies dramatically with the spreads $\sigma_{\theta}$ and $\sigma_{\phi}$ as illustrated by the upper curve in Figs. 12 .

\footnotetext{
${ }^{11}$ Angles are expressed with respect to the perpendicular to the bar face from which the particle enters in the quartz.
} 
Another way to proceed is to neglect correlations in the expression for $\chi^{2}$ (Rel. (14)) and solve at minimum. In this case, the result provides a fit value $R_{f i t}$ practically not biased. Its error $\sigma_{R}$ is computed from the solution at minimum $\chi^{2}$ and from the matrix $T^{-1}$ (see Rel. (16)) which gives the errors and correlations for $a, b, c$, from which one can deduce $\sigma_{R}$ using :

$$
\sigma_{R}^{2}=\frac{1}{4 R_{f i t}^{2}}<[\delta c+2 a \delta a+2 b \delta b]^{2}>
$$

and $R_{f i t}^{2}=c+a^{2}+b^{2}$, i.e. one takes into account the fact that the actual center is not the origin, but is closer to its fit value. The corresponding rms pull is the middle curve in Figs. 12. It shows already a much better behaviour than the previous method result which neglected all correlations stricto sensu. This full account of the fit center location mainly explains the interesting behaviour of the pull rms at large values for $\sigma_{\theta}=\sigma_{\phi}$.

Finally, the lowest curve in Figs. 12 shows the solution taking into account all correlations as explained in the sections above and in the appendices. In this case, the pull rms remains always close to 1 and departures are never worse than about $10 \%$ only["

Comparing the two Figs. 12, it is clear that the mean effect is much more dramatic if correlations due to multiple scattering are harder (at low mean momentum). It is also clear from these figures that there is a small systematic effect (10\% of the pull rms) that our model does not account for. The reasons for this systematic effect are several ; first, errors and correlations due to multiple scattering are treated statistically핑 second, at low momentum and/or at large angular errors on the incoming charged track direction, non-linear effects become visible. Figs. 12 show however that these effects remain of limited influence.

One may wonder why the second method which neglects correlations gives a pull rms which improves with large angular errors on the incoming charged track direction, a behaviour quite different from the simple uncorrelated mean (first of the methods above). This is actually due to the peculiar origin of this kind of correlations compared to multiple scattering. In Fig. 13, the pull rms is plotted as a function of $1 / p$ (i.e. for increasing angular errors dues to multiple scattering) ; crosses correspond to the second method, dots to the third (standard) method. It is clear herefrom that neglecting correlations gives a worse and worse result when multiple scattering errors increase.

The different behaviour of correlations due to multiple scattering only (case A) and to errors on the incoming track direction only (case B) can be understood to some extent. Let us assume that we are in case B ; if we have had an exact knowledge of the actual charged track direction, correlation effects would vanish when using it to subtract the center position from the photon coordinates. Using the fit center, in place of of the measured center (here the origin), improves somehow the approximation made of the actual charged track direction; this is well reflected in Figs. 12 by the difference of slope between the curves with square and cross markers. Instead, in case $\mathrm{A}$, there is clearly no longer one and only one actual charged track direction associated with the photon set, and consequently, neglecting correlations should degrade the result more and more as multiple scattering effects increase ; this is reflected by the behaviour shown in

\footnotetext{
${ }^{12}$ Actually, going to much larger values for $\sigma_{\theta}=\sigma_{\phi}$, the lowest curve remains at about 0.9 while the middle curve crosses 1 and goes on decreasing.

${ }^{13}$ We take into account only mean effects due to multiple scattering. Actually, the emission time sequence of the detected photons is not known and, moreover, the real multiple scattering effect undergone by the track before each photon is emitted is also unknown. Therefore, it seems hard to imagine how to go beyond averaging.
} 
Fig. 13 by the (cross) curve. Real life stays in between cases A and B, since actually we are in a mixed case.

In any case, as the "physical region" for $\sigma_{\theta}$ and $\sigma_{\phi}$ is expected to be around 1 to $2 \mathrm{mr}$, it is clearly preferable to account for all correlations. Depending on the mean track momentum, the pull rms departure from 1 may be as large as $\simeq 50 \%$ instead of the $\simeq 10 \%$ mainly due to non-linear effects. This problem is reflected by the $\chi^{2}$ probability distributions. Focusing on the mixed track sample with $0.5 \mathrm{GeV} \leq p \leq 5 \mathrm{GeV}$ and neglecting only the correlations in the $\chi^{2}$ (second of the methods just discussed), we get the distributions shown in Fig. 14. Here are displayed the probability distributions corresponding to various values for $\sigma_{\theta}=\sigma_{\phi}$ from $0-$ Fig. $14 \mathrm{a}$ - to $5 \mathrm{mr}$-Fig. 14 - compared with the case where all correlations are normally accounted for (Fig. 14f $)$, corresponding to $5 \mathrm{mr}$. We see that, already for small angular errors on the charged track direction, the probability distribution is badly distorted compared to flatness, while accounting normally for correlations gives an acceptably flat probability distribution ${ }^{\text {t4 }}$, even at $5 \mathrm{mr}$. One should also notice from Fig $14 \mathrm{f}$ that the systematic effects already noticed, which survives our treatment of errors and correlations at $\sigma_{\theta}=\sigma_{\phi}=5 \mathrm{mr}$ is not hard enough that it would spoil the shape of the distribution probability.

One thus has to notice the dramatic effect of a bad estimate of the center coordinates on the rms pull and then on probability distributions. This effect on the pull rms is actually due to a wrong estimate of the errors and correlations. Indeed, as noticed in Section 3 when discussing Rel. (4), this effect affects much less the central estimate of the radius and hence, of the Cerenkov angle than the error estimation itself.

\subsection{Track-Events with various Background Conditions}

Here we examine samples contaminated by various kinds of background : flatly distributed noise on the PMT detection plane, or merging of the track with one or sometimes two (unidentified or identified) tracks which produce additional photons. The chosen momentum range is still 0.5 to $5 \mathrm{GeV} / \mathrm{c}$ and the population contains the five possible particle kinds in equal numbers. This kind of background conditions can be considered the hardest as these additional tracks enter the same DIRC bar, sometimes with directions very close to that of the track under identification.

The pull of the Cerenkov angle for the sample of mixed particles with one identified additional track is plotted on Fig. 15a. This plot gives two important informations : on the one hand, the pull bias remains as small as when there is no background (see Fig. 8 $\mathrm{b}$ ) ; on the other hand, the pull rms is close to one (typically 1.2), as one expects if the error model is correct. A simple gaussian fit to the distribution in Fig. 8b gives $\sigma=0.95$ with a very good fit probability. All this shows that the tails to the distributions remain limited and do not affect the fit quality.

Figure 15]b, a plot of the difference $\theta_{C}^{\text {true }}-\theta_{C}^{f i t}$, allows to estimate the absolute dispersion of this quantity. Compared with the case with no background (see Fig. 8c), one sees clearly that the bias is unchanged and limited $(0.3 \mathrm{mr})$, whereas the dispersion is slightly increased $(3.6 \mathrm{mr}$

\footnotetext{
${ }^{14}$ The difference in flatness between Fig. ( $(8 \mathrm{a})$ and Fig (14f) is simply due to different median cuts : In the former case it was set at the very loose level of $4 \sigma$, while in the later it is $3 \sigma$. One can furthermore compare these two figures and remark that a tighter median cut mainly affects the low probability region by depopulating it somehow.
} 
instead of $3 \mathrm{mr}$ ).

This dispersion depends, among other things, of the track momentum and dip angle. With this respect, Fig. 16 shows the mean theoretical error on the reconstructed Cerenkov angle as a function of this momentum ; one can check that this error increases noticeably at low momentum (mainly because of multiple scattering effects). Errors become smaller and reach a minimum plateau at high track momentum because of the Cerenkov angle saturation, the greater number of Cerenkov photons (proportional to $\sin ^{2} \theta_{C}$ ), and smaller multiple scattering effects.

The fit results obtained when no background was added to the track were good (see Figs. 8, 9 and 10). They deteriorate only slightly if noise is added : Fig. 17 indicates that the algorithm still behaves well under background conditions connected with the presence of other (measured) tracks in the event. In Figs. 17b and 17k, the reconstructed Cerenkov angle pulls are displayed when one or two other tracks are superimposed to the signal track (their existence is known to the algorithm, which uses this knowledge to reject part of this background as ambiguities, as explained in Section 6.2.1) ; the dispersion and bias of the reconstructed angle are found close to the normal ones.

For the case of Figure $17 \mathrm{a}$, where flat background has been superimposed to the signal track, the algorithm seems to be more affected, since a sizeable bias of $15 \%$ appears, the rms dispersion increases but stays close to 1 . This bias still is limited, even if noise conditions are quite severe compared to what is expected with a real detector.

Fig. 18 shows the $\chi^{2}$ probability distribution for $n_{\gamma}-1$ degrees of freedom for an equal mixture of single electron, muon, pion, kaon and proton tracks. It illustrates the effects of the same cuts on different background conditions (the cuts used have been calibrated on events with only a flat background). The plots are shown for several cases of actual background conditions: a) no background, b) random flat background on the detection plane (at the level of $100 \%$ of the signal photons), c) 1 other track considered as background (i.e. no secondary $|\delta \theta|$ cut, no knowledge of the existence of the second track, see section 6.2.1).

Fig.18a shows that the actual effect of the cuts on background free events is mainly concentrated in the low probability bins which appear slightly depopulated, while the rest of the distribution looks acceptably flat. Figs. 18b and 18c show that the main effect of background photons is to provide a large peak at small probability, and thus the need for a threshold probability. These last two figures tends also to show that a large flat background is harder to account for than the background associated with photons generated by an unmeasured track : in the former case, the low probability peak extends up to $\simeq 15 \%$, while in the later case the effect of this peak is negligible above $\simeq 5 \%$. This kind of plots, which can be produced with any data set, are tools allowing to tune the minimum probability above which the fit of the Cerenkov ring is considered.

As a global check, Fig. 19 shows a plot of the $\chi^{2}$ per number of degrees of freedom obtained after running the procedure on the same sample of single tracks for several noise environments : a) without any background, b) with a random flat background, c) with several tracks per event considered as background for the original track. One can see that after the cut adjustment, 
the mean $\chi^{2}$ per ndof is close to 1 . The rms of the $\chi^{2}$ per ndof distribution behaves also as expected, for example in the case (a) the mean is 0.96 and the rms of the distribution ( 0.32 for

a mean number of degrees of freedom of 21$)$ is effectively close to $\sqrt{\frac{2}{n d o f}}(0.31)$. The number of reconstructed events varies between the three plots because of different cut levels adapted to different noise conditions.

\subsection{Particle Identification}

The final goal of pattern recognition and circle reconstruction is particle identification. In order to illustrate how the procedure behaves, we present results obtained for one track, assuming another track has crossed the same (and single) bar. The angle between the two tracks is random between $0^{\circ}$ and $50^{\circ}$.

In Fig. 20 we show the reconstruction of generated pions and present (in a)) the case when the accompanying track has been reconstructed and the secondary cut on $|\delta \theta|$ has been applied. In (b), we assume this track has not been measured and then one cannot use this further cut. In both cases, no threshold probability has been required and the identification is attributed to the largest probability (which can thus be quite small). The correct identification (except for $e^{-} \mu-\pi$ degeneracy) is good, above $90 \%$ anyway. One can further see that the secondary cut on $|\delta \theta|$ produces a large improvement by reducing severely the misidentification of pions as kaons and protons. Despite the $e-\mu-\pi$ degeneracy, below $\simeq 950 \mathrm{MeV}$ pion misidentification as electron looks negligible and, then, electron identification below this threshold is possible with a good accuracy. Misidentification is low below $\simeq 3.5 \mathrm{GeV} / \mathrm{c}$.

In Fig. 20c, we have applied both the secondary cut on $|\delta \theta|$ and a threshold probability of $1 \%$ which cleans up almost completely the momentum range below $\simeq 3.5 \mathrm{GeV} / \mathrm{c}$. It is easy to see that, strengthening this threshold to $3 \%$, sharply reduces the misidentification which becomes significant only above $\simeq 4 \mathrm{GeV} / \mathrm{c}$.

All this illustrates that the largest difficulties are due to unmeasured tracks or unstructured noise. As soon as a limited additional information is available (secondary track directions), the quality of the identification sharply improves. Setting a threshold probability at a low level appears naturally to be a suitable cleaning up tool, as a non-negligible part of the misidentification, and hence of contamination, is produced by low probability reconstructions.

\subsection{Summary}

As a matter of conclusion, even under severe background conditions, it is possible to remove background and select photons associated with a given charged track with a good efficiency (still about $90 \%$ of the tracks are fit) and with a limited contamination (about $95 \%$ of particles identified as pions are indeed pions and the misidentification as kaons is always low, sometimes very low). This is achieved by starting with using the subset of unambiguous photons ; indeed fitting with them the Cerenkov ring gives access to refined values of the circle parameters which are used when reexamining the ambiguous photons. These improved parameter values allow a refined treatment of the photons left aside as ambiguous.

The procedure described in Section 6 could possibly be improved, but basically it contains most of the needed ingredients. Moreover, the fit algorithm presented in Section 4 looks well adapted in order to fit circle arcs and to provide $\chi^{2}$ distances for fake photon removal. This 
method allows to use all basic statistical tools (probabilities, likelihoods ...). Of course, we know that fake photons cannot be removed at the $100 \%$ level, however, we have proved that their residual contamination, in severe enough conditions, can be made low enough that the fit quality and its statistical meaning are not spoiled.

\section{Cut Handling}

The various cuts described in Section 6 represent finally a set of 8 parameters : two cuts on $|\delta \theta|$ allowing to define the starting sample of unambiguous photons, the median cut level for background removal, the "perpendicular tracks" cut used to remove trivial ambiguities, the two $\chi^{2}$ distance cuts in the ambiguous photons recovering step, the minimum number of unambiguous photons, and the tails removal cut after the first fitting step.

Some of these cuts can be adjusted in order to adapt the algorithm to different noise and background conditions, some are nearly fixed by the detector characteristics $(|\delta \theta|<30 \mathrm{mr}$, perpendicular track cut), or by algebra (the minimum number of unambiguous photons is set at the smallest admissible value). Also, one can verify that the last two cuts only influence marginally the reconstruction procedure performance.

The $3 \chi^{2}$ cuts have a well defined statistical meaning and the procedure outputs usual $\chi^{2}$ probabilities. Simple criteria can be used in order to adjust these cuts, in such a way that they filter the background without spoiling too much the signal. Indeed, too stringent cut values may bias dramatically the fit quantities or spoil the probability density function of the fit results. Too loose cuts may instead result in very low reconstruction efficiency and poor fit quality. As, in the DIRC problem, errors are close to gaussians, $3 \sigma$ in tuning these cuts is a magic value of well defined meaning. When having to cut below this level one has to check for potentially harmful effects (biases or unacceptable changes in the probability density function of the fit results).

\subsection{Tuning of the Adjustable Cuts}

In fact, only the first three parameters in the preceding list can be considered as adjustable by the user to adapt the algorithm to different noise and background conditions.

As illustrated above, the main tool in tuning the various cut levels is the probability distribution of reconstructed rings. From the most common experience, one knows that if errors and correlations are well understood, these distributions are flat between 0 and 1; one can always assume this has been checked on clean samples. Clean samples can indeed be constructed using a Monte Carlo. Tagged samples of identified (by other means) particles, or low multiplicity samples, can always be extracted from data.

Then, once the reliability of the error/correlation handling is ensured, any departure from flatness has to be attributed to background. The set of cuts defined above is devoted to the removal of the various kinds of background photons. They are tuned by asking the particular value of the cut to optimize the flatness of the probability distribution. Ideally, when all cuts are at optimum values, the probability distribution has mean value 0.5 and rms 0.289 .

In real life however, one knows that an actually flat distribution always exhibits a peak at low probability, reflecting the fact that there always exist configurations where events are 
improperly reconstructed. This situation has been already met here (see Figs. 18). Therefore the above rules have to be slightly modified. Flatness has to be requested above some threshold value $\alpha$, which can be relatively large for the purpose of tuning ( $\alpha=10 \%, 20 \%$ or more are as good). One can always compute the mean value and rms of the probability distribution above this threshold as a function of the cut value and fit it (or compare) to respectively $(1-\alpha) / 2$ and $(1-\alpha) / \sqrt{12}$.

Fig. 21, which deals with a sample of single tracks with no additional background, illustrates how this tuning can be performed for the median cut. When fixing the cut level at $1 \sigma$, we are clearly cutting too tight and then the probability distribution exhibits a huge peak at 1 . Releasing this cut allows to recover a non-biasing behavior at a cut level of about 3 to $4 \sigma$ as one can expect : the mean probability is found close to 0.5 and its rms close to $1 / \sqrt{12}$. One can refine the tuning in an obvious manner.

One may notice that the number of entries in the histograms is only marginally dependent on this cut level : the algorithm efficiency is not directly affected by it (but the final errors on the fitted quantities are changing with this cut - roughly like $\frac{1}{\sqrt{n_{\gamma}}}-$ since the total number of unambiguous photons entering the fit is cut dependent, even if the recovering procedure finally limits its variation).

On the other hand, the low probability peak, as said above, reflects the existence of improperly reconstructed rings. This is partly due to configurations where the errors are not well estimated[t] and partly due to the level of background which survives the cuts. Usually, such a peak is made harmless by setting a threshold probability.

The two $|\delta \theta|$ cut play an important role in the magnitude of this peak. The primary $|\delta \theta|$ cut, if it happens to be too loose, accepts more easily background photons in the unambiguous photon subsamples. These background photons will in turn degrade the fit quality and contribute to increase the peak at zero probability. However, it cannot be set too tight (for instance $\leq 5 \mathrm{mr}$ ) since in this case, the number of unambiguous photons primarily associated with tracks may decrease too much (below 2), and consequently the number of tracks lost will increase without a significant improvement at the zero probability peak.

The secondary $|\delta \theta|$ cut acts likewise as can be seen from Fig. 22. Its strong power of rejecting the inter-track noise is illustrated by the vanishing low probability peak in quite hard background conditions.

These examples illustrate how a given cut level can be tuned on any sample of tracks ; here again the procedure applies to simulation and real data as well. The cut levels are tuned in such a way that the probability distribution has its expected flat shape. This tuning allows to recover at the same time a mean probability of $\simeq 0.5$ and a spread of $\simeq 1 / \sqrt{12} \mathrm{rms}$. After discarding (eventually) the low probability bins, it is easy to compute the efficiency of any further cuts in probability, because of the flatness of the remaining region.

\subsection{Effect of non tunable cuts}

The non tunable cuts, briefly considered before, are the perpendicular tracks recovering cut and the minimum number of unambiguous photons cut. The former is tunable only in the sense

\footnotetext{
${ }^{15}$ In the DIRC problem, the final errors are computed by differentiating functions. They are geometry dependent and sometime close to singular points of these functions.
} 
that it should be adjusted on data or on Monte Carlo simulation not to spoil the algorithm acceptance (eventually creating biases if the cut is too wide), and not being rendered inefficient by a too stringent width (in this case, only a small fraction of the almost perpendicular tracks is concerned by the cut). Anyway, as stated before, this cut has only limited effects : its activation or deactivation is hardly noticeable on the studied data.

The second non adjustable cut has a rather strong influence on the global algorithm acceptance. Figure 23 shows the distribution of the number of unambiguous photons at the end of the procedure : it is clear that if the cut level is increased starting from the normal value of 2 , the number of non accepted tracks will be strongly affected, since in this region the distribution is showing a strong dependence of the number of tracks upon the number of unambiguous photons. Normally, there is no point in increasing the cut level above 2 or 3 unambiguous photons.

To conclude this section, we can say that the choice of the cut level for both cuts is not really free, but constrained by considerations depending strongly on the use of the algorithm and the type of data.

\section{Conclusions}

We have studied a procedure able to perform pattern recognition among photons in order to reconstruct the Cerenkov angle associated with the charged track emitting them. The procedure, which has been developed for the case of the DIRC, may apply mutatis mutandis to data from other ring imaging devices. The basic requirement was that the procedure should provide a good approximation of a $\chi^{2}$ value in order that a probability can be acceptably defined, even in presence of a huge background mixed to the signal photons. We have shown that such a procedure can indeed be constructed and proved to work satisfactorily from the kaon Cerenkov threshold up to $4 \div 5 \mathrm{GeV} / \mathrm{c}$.

We advocated the use of the stereographic projection which guarantees that the figure to be fit is always a circle, whatever systematic errors, misalignment problems, etc ... are. Moreover, we have shown that such kind of errors affects negligibly the estimate of the central value for the radius ( i.e. the Cerenkov angle).

The basic tool of the procedure is a fit corresponding to minimising a $\chi^{2}$ expression, linearised as commonly done. In the case of the DIRC, this $\chi^{2}$ has to be modified in order to take into account that the photon do not populate the full circle, but rather a relatively small fraction of it. The modification implemented turns out to take into account in the fit procedure the existence of a measurement of the charged track direction, beside the photon directions. In this way, systematic errors in the circle reconstruction can be avoided almost completely. We have also widely illustrated the role of correlations and methods to estimate them. The fit procedure returns a value of the circle radius (connected with the Cerenkov angle) and an improved information on the charged track direction. This last information has been shown to be crucial in cases where the charged track direction is poorly known, either intrinsically (from the tracking device in front of the DIRC), or because of large multiple scattering effects.

We have shown, that using this tool, it is possible to define an algorithm able to solve 
ambiguities and remove efficiently background photons. It relies on an iterative procedure, based on an intensive use of $\chi^{2}$ distances. It starts with unambiguous photons and recovers additional photons in a second pass. Such a procedure depends on cuts, and it has been shown that one can check the effects of these cuts and tune their levels on pull and probability distributions. This allows also to define a calibration procedure which can be worked out with real data in a simple way and optimized in real background conditions.

With this respect we have shown that the "combinatorial" background generated by ambiguities can be easily overcome. We have also shown that the background produced for a given track by photons associated with other reconstructed tracks was easy to deal with. The hardest background is provided by non-reconstructed tracks or flat background of various origins ; we have shown that it is possible to deal reliably with them too, by using tighter cut levels.

As a final result, we have shown that the reconstruction and particle identification are possible through a fit of the Cerenkov angle and particle direction, with a remarkable efficiency (above $90 \%$ ). We have also shown that the fit probability is correctly estimated and has the expected flat distribution. This means that the cleaning up part of the procedure is able to discriminate efficiently between hits, even when there is a large background together with the signal. The residual background contamination level has been shown to be harmless under realistic conditions. Therefore one can use standard statistical tools in order to calibrate cuts and check the reconstruction quality. 


\section{Appendix A : Errors and Correlations in a DIRC Device}

\section{A1 Error Functions of the Charged Track Direction}

The charged track direction is affected by two different kinds of errors. The first kind are the measurement errors on the track at the entrance into the radiator (the quartz bar), the second is the error produced by its multiple scattering inside the radiator, which makes that, for each emitted photon, the Cerenkov angle $\theta_{C}$ is relative each time to a slightly modified track direction. Let us denote the incoming track direction by $\vec{q}=(\sin \theta \cos \phi, \sin \theta \sin \phi, \cos \theta)$; the condition $\vec{q}^{2}=1$ implies that $\vec{q} \cdot \delta \vec{q}=0$, and then that the error vector $\delta \vec{q}$ is perpendicular to the track direction $\vec{q}$. Let us write it :

$$
\delta \vec{q}=\delta_{1} \vec{q}+\delta_{2} \vec{q}
$$

where $\delta_{1} \vec{q}$ refers to the measurement errors and $\delta_{2} \vec{q}$ to the multiple scattering. The error functions on the track parameters $(\theta, \phi)$ being denoted $(\delta \theta, \delta \phi)$, we have :

$$
\delta_{1} \vec{q}=\delta \theta \vec{v}+\sin \theta \delta \phi \vec{w}
$$

where $\vec{v}=\partial \vec{q} / \partial \theta$ and $\vec{w}=[1 / \sin \theta] \partial \vec{q} / \partial \phi$ are unit vectors orthogonal to each other and to $\vec{q}$. We may have $<\delta \theta \delta \phi>\neq 0$. After a path of length $u$ inside the quartz, we also have :

$$
\delta_{2} \vec{q}=c \sqrt{\frac{u}{X_{0}}}\left[\varepsilon_{1}(u) \vec{v}+\varepsilon_{2}(u) \vec{w}\right]
$$

where [16] $c=13.610^{-3} / \beta p[\mathrm{GeV}], \beta$ is the particle speed and $X_{0}$ is the quartz radiation length. The quantitites $\varepsilon_{i}(u)$ are gaussian random variables such that $\left.<\left[\varepsilon_{i}(u)\right]^{2}\right\rangle=1$ and $<\varepsilon_{1}(u) \varepsilon_{2}(u)>=0$. Here and throughout the paper we neglect all departures from gaussian distributions [16]. From Rel. (A.3), we get :

$$
<\left[\delta_{2} \vec{q}\right]^{2}>=2 c^{2} \frac{u}{X_{0}}
$$

However, as we don't know where the photon has been emitted, the best estimate of $<\left[\delta_{2} \vec{q}\right]^{2}>$ for this variance is its mean value over the path followed, assigning an equal probability to each possible emission point $\square$ :

$$
<<\left[\delta_{2} \vec{q}\right]^{2}>>_{u}=\frac{1}{L} \int_{0}^{L} 2 c^{2} \frac{u}{X_{0}} d u=c^{2} \frac{L}{X_{0}}
$$

where $L$ is the total path length of the charged particle inside the quartz.

Let us assume that two photons are emitted after respectively paths $u_{1}$ and $u_{2}$ inside the quartz. As the photon detection does not reveal where it has been emitted, we can have with equal probabilities $u_{1}>u_{2}$ or $u_{2}>u_{1}$. Therefore, up to higher order corrections, we have :

\footnotetext{
${ }^{16}$ The notation here is obvious : the inner $<\cdots>$ denotes the statistical mean (i.e. the expectation value) already defined in the body of the text, while the outer $\left\langle\cdots>_{x y} \ldots\right.$ denotes the additional average performed over continuous variables $x, y, \cdots$.
} 


$$
\left\{\begin{array}{l}
\text { if } \quad u_{2}>u_{1}:\left\{\begin{array}{l}
\delta_{2} \vec{q}\left(u_{1}\right)=c \sqrt{\frac{u_{1}}{X_{0}}}\left[\varepsilon_{1}\left(u_{1}\right) \vec{v}+\varepsilon_{2}\left(u_{1}\right) \vec{w}\right] \\
\delta_{2} \vec{q}\left(u_{2}\right)=\delta_{2} \vec{q}\left(u_{1}\right)+c \sqrt{\frac{u_{2}-u_{1}}{X_{0}}}\left[\varepsilon_{3}\left(u_{2}\right) \vec{v}+\varepsilon_{4}\left(u_{2}\right) \vec{w}\right]
\end{array}\right. \\
\text { if } \quad u_{1}>u_{2}:\left\{\begin{array}{l}
\delta_{2} \vec{q}\left(u_{2}\right)=c \sqrt{\frac{u_{2}}{X_{0}}}\left[\varepsilon_{1}\left(u_{2}\right) \vec{v}+\varepsilon_{2}\left(u_{2}\right) \vec{w}\right] \\
\delta_{2} \vec{q}\left(u_{1}\right)=\delta_{2} \vec{q}\left(u_{2}\right)+c \sqrt{\frac{u_{1}-u_{2}}{X_{0}}}\left[\varepsilon_{3}\left(u_{1}\right) \vec{v}+\varepsilon_{4}\left(u_{1}\right) \vec{w}\right]
\end{array}\right.
\end{array}\right.
$$

where the various $\varepsilon$ 's carry unit variance and are statistically independent when they carry different indices and/or different arguments (i.e. $<\varepsilon_{i}\left(u_{j}\right) \varepsilon_{k}\left(u_{l}\right)>=0$ only if $i \neq k$ and/or $j \neq l)$.

One can check that $\delta_{2} \vec{q}\left(u_{2}\right)$ and $\delta_{2} \vec{q}\left(u_{1}\right)$ have the same variance given by Rel. (A.4 (or by (A.5) and it is shared equally between their $\vec{v}$ and $\vec{w}$ components (remind that $\vec{v} \cdot \vec{w}=0$ ). Moreover, we can now compute the expectation value :

$$
<\delta_{2} \vec{q}\left(u_{1}\right) \cdot \delta_{2} \vec{q}\left(u_{2}\right)>=<\left[\delta_{2} \vec{q}\left(u_{1}\right)\right]^{2}>\Theta\left(u_{2}-u_{1}\right)+<\left[\delta_{2} \vec{q}\left(u_{2}\right)\right]^{2}>\Theta\left(u_{1}-u_{2}\right)
$$

where $\Theta$ is the standard step function and the expectations values on the RHS are given by Rel. (A . 4) with the appropriate argument. It is useful to have an estimate of this correlation coefficient ; it is obtained by averaging Rel. (A.7) upon $u_{1}$ and $u_{2}$. This is easily achieved:

$$
<<\delta_{2} \vec{q}\left(u_{1}\right) \cdot \delta_{2} \vec{q}\left(u_{2}\right)>>{ }_{u_{1} u_{2}}=\frac{1}{L^{2}} \int_{0}^{L} \int_{0}^{L} d u_{1} d u_{2}<\delta_{2} \vec{q}\left(u_{1}\right) \cdot \delta_{2} \vec{q}\left(u_{2}\right)>=\frac{2}{3} c^{2} \frac{L}{X_{0}}
$$

Therefore, the average correlation amounts to $2 / 3$ of the average variance. The covariance fraction carried by each component are :

$$
\left\{\begin{array}{l}
<<\left[\delta_{2} \vec{q}\left(u_{1}\right) \cdot \vec{v}\right]\left[\delta_{2} \vec{q}\left(u_{2}\right) \cdot \vec{v}\right]>>{ }_{u_{1} u_{2}}=\frac{1}{3} c^{2} \frac{L}{X_{0}} \\
<<\left[\delta_{2} \vec{q}\left(u_{1}\right) \cdot \vec{w}\right]\left[\delta_{2} \vec{q}\left(u_{2}\right) \cdot \vec{w}\right]>>_{u_{1} u_{2}}=\frac{1}{3} c^{2} \frac{L}{X_{0}}
\end{array}\right.
$$

while all other covariance mean values are zero.

\section{A2 Finite Size Sample Corrections to Multiple Scattering Errors}

It is clear that the length of the path followed before the track emits any photon is inaccessible, nor the ordered time (or path) sequence of the detected photons. This implies that one has to work with averaged quantities. In the previous subsection, averaging is defined by Rels. (A.5) and (A.8), for respectively the variance and covariance terms. Averaging by integrals assumes the emission of an infinite number of photons along the charged track path inside the radiator.

Here we present another method, based on a finite number of radiated photons ; it allows to find the $1 / n$ corrections to the above method, while checking it conceptually. 
Let us assume $n$ detected photons are emitted along the path of length $L$. Photon acceptance is mainly connected with their azimuth on the Cerenkov cone ; therefore we can assume for simplicity, that these photons are emitted after equal paths of length $L / n$. Let us also assume we work with each coordinate of the circle center in the equatorial plane (final results for variances have to be multiplied by 2 for comparison with the preceding subsection). We denote by $x_{i}$ the coordinate of the charged track direction (actually its fluctuation around $x_{0}$, the true

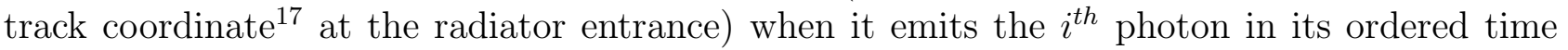
sequence. Then we have :

$$
\left\{\begin{array}{l}
x_{1}=x_{0}+\epsilon_{1} \\
x_{2}=x_{0}+\epsilon_{1}+\epsilon_{2} \\
x_{3}=x_{0}+\epsilon_{1}+\epsilon_{2}+\epsilon_{3} \\
\ldots
\end{array}\right.
$$

where the functions $\epsilon_{k}$ are centered independent random variables : $\left\langle\epsilon_{k}>=0\right.$ and $\left.<\epsilon_{k} \epsilon_{l}\right\rangle=$ $\sigma^{2} \delta_{k l}\left(\sigma^{2}=c^{2} L /\left(n X_{0}\right), c\right.$ being already defined). Let us also define $A=n \sigma^{2}$, a quantity

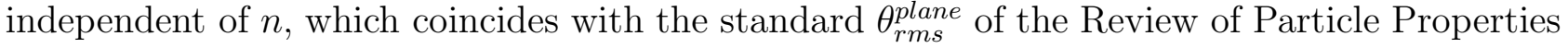
[16. For simplicity, we choose from now on $x_{0}=0$. Trivially, writing $\left\langle\epsilon_{k}\right\rangle=0$ for all $k$, does not mean it is true for any given track, but that this is fulfilled by the mean values computed from a large sample of tracks. Indeed, one set of $x_{i}$ corresponds to one track and then to one sampling of the $\epsilon_{k}$ 's.

The ordered time sequence is unknown ; nevertheless, we can define the multiple scattering variance of the sample by :

$$
V_{m}=\frac{1}{n} \sum_{k} x_{k}^{2}
$$

Using Rels. (A . 10, it is easy to find the expectation value of $V_{m}$ :

$$
<V_{m}>=\frac{n+1}{n} \frac{A}{2}
$$

which tends to half the value in Rel. (A.5), when $n \rightarrow \infty$, as expected. Here we have to split up the result into two parts (correlated and uncorrelated). The correlated part is the variance of the center of gravity :

$$
G=\frac{1}{n} \sum_{k} x_{k}
$$

It is easy to compute it and get :

$$
<G^{2}>=\frac{(n+1)(2 n+1)}{2 n^{2}} \frac{A}{3}
$$

\footnotetext{
${ }^{17}$ Obviously, the true coordinate at the DIRC entrance is approximated by the mean value provided by the tracking device in front of the DIRC, but does not coincide with it.
} 
which tends to $2 / 3$ of $\left\langle V_{m}>\right.$ when $n \rightarrow \infty$, in agreement with Rel. (A. 8). The mean uncorrelated part (reduced variance of the sample) is the mean value of :

$$
W_{m}=\frac{1}{n} \sum_{k}\left(x_{k}-G\right)^{2}
$$

which is :

$$
<W_{m}>=\frac{n^{2}-1}{n^{2}} \frac{A}{6}
$$

and tends to $1 / 3$ of the variance when $n \rightarrow \infty$. Therefore, the integral averaging presented in the previous subsection gives indeed the large $n$ behaviour of the mean multiple scattering effects. The subleading terms are of the order $1 / n$ and small ; they can easily be read off the results in this subsection. They show that the sharing uncorrelated-correlated parts of the variance departs from $1 / 3: 2 / 3$ by terms of the order $1 /(3 n)$. They may become important only for number of photons of the order $n \leq 4 \div 5$.

\section{A3 Handling of Other Errors}

There are qualitatively two kinds of errors we have to deal with in the DIRC problem. The first kind are mostly geometric errors due to the reconstruction of the photon direction : finite size of the photomultipliers, finite size of the bar entrance window. There is no difficulty of principle in their reconstruction and propagation from the water tank and photomultipliers back into the quartz bar and we will not comment on this any longer.

The second kind of errors (chromaticity) is due to the dependence of the refraction indices (water and quartz as far as the DIRC is concerned) upon the photon wavelength. This is due to the fact that photons emitted by Cerenkov effect do not have a definite wavelength, which rather runs over a relatively wide spectrum.

When refracting back the photon direction from water (index $n_{W}(\lambda)$ ) to quartz (index $n_{Q}(\lambda)$ ), it is appropriate to consider as basic random variable the quartz index ; then, the ratio $g$ of these indices can be expressed as a function of $n_{Q}$. In the BaBar setup, this ratio can be considered constant over the range of wavelengths to which the photomultipliers are sensitive.

The quartz index is surely an appropriate variable because, thanks to Rel. (囵), it affects directly the expected Cerenkov angle $\theta_{C}$ and its error for each photon. This is an important source of errors (it turns out to be equivalent to treating the index as a random variable with a standard deviation corresponding to $\delta n / n \simeq 0.6 \%$ ). This error can be either attributed to the charged track direction or to each photon direction separately, but, in the former case, one has to take into account the fact that these photon errors are uncorrelated (this gives rise to corrections in the error magnitude once applied globally to the track direction ). In the course of the fit procedure, the $\chi^{2}$ which allows to reconstruct the circle radius takes naturally this effect into account. Moreover, when fixing the Cerenkov angles to the values expected from the charged track momentum, assuming the possible mass assignments (the consistency check referred to in the body of the text), it allows to take into account automatically the spread due to the photon wavelength spectrum. 


\section{Appendix B : Basics for a Circle Arc Reconstruction Al- gorithm}

As noted in the main text, when fitting a circle having at hand points (subject to measurement errors) spread out onto a relatively small arc (typically $60^{\circ}$ ), and if the error on each point is non-negligible compared to the radius of the circle 18 , the fit quality becomes poor.

Indeed, one can attempt a circle fit using standard algorithms [11, 12, 13]. However, fitting the 3 circle parameters under the typical conditions sketched above, using only the points on the circle leads to unexpected bad results. The center coordinate in the direction associated with the two outmost "experimental" points is relatively good ; however, the center coordinate in the direction toward the arc is significantly and systematically displaced towards the arc (compared with its expected value) and consequently the fit radius is systematically underestimated, sometimes badly.

One way to circumvent this problem is to introduce additional information about the circle center (the charged track direction as measured by another device than the DIRC, for instance a drift chamber). In this case, the fit algorithm works much better (as illustrated in this paper) in the sense that the pull is found with the expected unit standard deviation and a negligible residual methodological bias ${ }^{\text {TP }}$.

\section{B1 A Naive Estimate of the Error Matrix of the Charged Track}

The vector $\delta \vec{q}$ defining the error on the charged track direction $\vec{q}$ is given by Rels. (A. 1, (A.2) and (A.3). It is perpendicular to the charged track direction, and it projects out onto the equatorial plane (through the stereographic projection) as $\delta \vec{q} / 2$. Denoting $a$ and $b$ the coordinates of the circle center in the equatorial plane in the directions parallel resp. to $\vec{v}$ and $\vec{w}$ (both orthogonal to $\vec{q}$ ), we can define the error functions on the circle center by :

$$
\left\{\begin{array}{l}
\delta a=\frac{1}{2} \delta \theta+\frac{1}{2} c \sqrt{\frac{u}{X_{0}}} \varepsilon_{1}(u) \\
\delta b=\frac{1}{2} \sin \theta \delta \phi+\frac{1}{2} c \sqrt{\frac{u}{X_{0}}} \varepsilon_{2}(u)
\end{array}\right.
$$

where $u$ is the path length followed by the charged track inside the radiator. No average value over the path length has to be performed in this naive approach. Doing this way, the error matrix $\Sigma$ for the charged track can be computed by taking the expectation value of appropriate second order terms. One can then choose, as a rule of thumb, to approximate the error functions above by considering the standard deviations at $u=L / 2$, i.e. at half the full path of the charged track inside the radiator ; in this case, we have :

\footnotetext{
18 With this respect a ratio of $2 \%$, typical for the BaBaR DIRC, is large when points are on a circle arc of about $60^{\circ}$. For a track fit in a drift chamber, $60^{\circ}$ is relatively large as the ratio of error to radius is here of about $10^{-3}$.

${ }^{19}$ Practically, the residual methodological bias is of the order $10^{-4}$ of the radius (i.e. about 0.1 to $0.2 \mathrm{mr}$ for the Cerenkov angle) and then it is overwhelmed by the bias originating from fake photons, which cannot be completely removed by any realistic procedure.
} 


$$
\text { rough estimates : }\left\{\begin{array}{l}
<[\delta a]^{2}>=\frac{1}{4}\left[<[\delta \theta]^{2}>+\frac{1}{2} c^{2} \frac{L}{X_{0}}\right] \\
<[\delta b]^{2}>=\frac{1}{4}\left[\sin ^{2} \theta<[\delta \phi]^{2}>+\frac{1}{2} c^{2} \frac{L}{X_{0}}\right] \\
<\delta a \delta b>=\frac{1}{4} \sin \theta<\delta \theta \delta \phi>
\end{array}\right.
$$

where the quantities $<[\delta \theta]^{2}>,<[\delta \phi]^{2}>$ and $\langle\delta \theta \delta \phi>$ are the elements of the error matrix $\Sigma_{0}$ provided by the reconstruction procedure from the tracking device in front of the DIRC bar. The question now is to get a motivated estimate of the full error matrix $\Sigma$ of the charged track direction by taking into account theoretical a priori information on multiple scattering.

\section{B2 First Approach to the Charged Track Error Problem}

Let us consider only the $a$ coordinate of the circle center, or restrict our problem to a one dimensionnal aspect. Solving the 2-dimensional case $(a, b)$ will follow straightforwardly.

Actually, what is of relevance for our problem, is the mean value and error on the directions of charged tracks associated with the emitted and detected photons. There is some difference with the errors at half path inside the radiator, as will be shown below.

For a charged track entering the radiator medium, the tracking device provides a measurement of the direction with its error matrix ( $\Sigma_{0}$ referred to above). Let us associate with this measurement the origin in the equatorial plane where all directions are projected out.

When the charged track emits photon $i$, it has a given (even if unknown exactly) direction ; this direction varies from photon to photon simply because of the multiple scattering the charged track undergoes. The intersection of this direction with the unit sphere has for image in the equatorial plane the point of coordinate $a_{i}$ along the direction $\vec{v}$ (see Section A1) ; what is of relevance for the Cerenkov angle estimate is clearly the mean value and standard deviation of the set $\left\{a_{i} ; i=1, \cdots n\right\}$.

On the other hand, even in perfect cases (no multiple scattering) the actual direction of a given track is obviously not the mean value (defined here as the central value given by the drift chamber reconstruction). Fitting the Cerenkov cone allows possibly to improve this last estimate using a (large) number of additional informations (photons), going thus closer to its actual value. The effect of multiple scattering is that the actual center seen from each photon is different and randomly distributed ; then we cannot access one actual circle center, but only define a mean value of the set of actual centers.

Moreover, if photons can allow for improving the average estimate of the charged track direction, errors can be estimated from the (underlying) parent random distribution.

Therefore, we can write :

$$
a_{i}=a_{0}+\delta a_{i} \quad, \quad i=1, \cdots n
$$

where $a_{0}$ denotes here the expectation value of the center of gravity of the $\left\{a_{i}\right\}$ set20 and where the random error functions $\delta a_{i}$ given by Rel. (B . 1) with different paths $u_{i}$, are unbiased (<

\footnotetext{
${ }^{20}$ Actually, this turns to define the $a_{i}$ 's by relations like Rels. (A .10), with an additional offset $a_{0}$ in place of $x_{0}$.
} 
$\left.\delta a_{i}>=0\right)$. In connection with what said just above, it should be stressed that the expectation value $a_{0}$ for all measured photons associated with one track is not necessarily zero, but the set of all $\left\{a_{0}\right\}$ (each associated with a given track) is surely distributed around zero, with known deviations. Then, what is of relevance in our problem is the value of $a_{0}$ and its standard deviation on a track by track basis.

In order to get the mean value and the error of the direction set $\left\{a_{i}\right\}$, one can minimize the function (we define a vector $g$ of components $g_{i}=1, \forall i=1, \cdots n$ in order to match indices) :

$$
F(a)=\left(a g_{i}-a_{i}\right) V_{i j}^{-1}\left(a g_{j}-a_{j}\right) \quad \text {, with } \quad V_{i j}=<\delta a_{i} \delta a_{j}>
$$

where summation over repeated photon indices is understood. The zero of $d F(a) / d a$ gives this minimum (using also Expression (B.3)) :

$$
a=\frac{g_{i} V_{i j}^{-1} a_{j}}{g_{i} g_{j} V_{i j}^{-1}}=a_{0}+\frac{g_{i} V_{i j}^{-1} \delta a_{j}}{g_{i} g_{j} V_{i j}^{-1}}
$$

This expression gives the usual result for the estimate $a$ from a set of measurements $\left\{a_{i}\right\}$ in the least squares approach. Its expectation value $\langle a\rangle=a_{0}$ is unbiased and its error function $\delta a$, which can be read off Rel. (B.5), allows to compute its standard deviation $\sigma_{a}$ $\left(\sigma_{a}^{2}=<[\delta a]^{2}>\right):$

$$
\frac{1}{\sigma_{a}^{2}}=g_{i} g_{j} V_{i j}^{-1}
$$

When there is no correlation $\left(V_{i j} \simeq \delta_{i j}\right)$, this expression gives the usual result $\left(\sigma_{a}^{-2}=\right.$ $\left.\sum_{i} \sigma_{a_{i}}^{-2}\right)$.

In general $\delta a_{i}$ and $\delta a_{j}$ are given by expressions like in Rels. (B . 1) with two different path lengths $u_{i}$ and $u_{j}$, both unknown. Correspondingly the quantity $V_{i j}=<\delta a_{i}\left(u_{i}\right) \delta a_{j}\left(u_{j}\right)>$ can only be estimated by performing the average as presented in Section A1 of Appendix A. This gives :

$$
V_{i j}=<<\delta a_{i}\left(u_{i}\right) \delta a_{j}\left(u_{j}\right)>>_{u_{i} u_{j}}=\left[B E+\frac{1}{24} A I\right]_{i j}
$$

where $E$ is a rank 1 matrix such that each $E_{i j}=1$; here $B=\left[<[\delta \theta]^{2}>+1 / 3 A\right] / 4$ and $A=c^{2} L / X_{0}$. In order to compute $\sigma_{a}^{2}$ using Rel. (B.6), we need to invert $V$ just defined. Indeed, using $E^{2}=n E$, it is easy to prove that :

$$
V=\lambda I+\mu E \Longleftrightarrow V^{-1}=\frac{1}{\lambda}\left[I-\frac{\mu}{n \mu+\lambda} E\right]
$$

where $n=\operatorname{dim} I=\operatorname{dim} E$ is also the number of points (photons). Using this formula with the matrix in Rel. (B.7), we easily find :

$$
\sigma_{a}^{2}=\frac{1}{4}\left[<[\delta \theta]^{2}>+\frac{1}{3} A+\frac{1}{6 n} A\right]
$$

\footnotetext{
${ }^{21}$ As all $a_{i}$ have been fixed at zero (the measured central value) for the track considered, this corresponds to take as sampling $\delta a_{i}=-a_{0} g_{i}$.
} 
This relation could be expected beforehand. It shows that the uncorrelated part of the error affecting each $a_{i}$ (2/3 of the variance associated with multiple scattering as shown by Rel. (A . 8), plus the variance provided by the device in front of the DIRC bar) is transfered to $a$ without changes, while the correlated part (1/3 of the multiple scattering contribution to the full variance) scales with $n$. If we had neglected the terms generated by the multiple scattering effects in $V_{i j}$ (for $i \neq j$ ), we would have got instead $\sigma_{a}^{2}=\left[<[\delta \theta]^{2}>+A /(2 n)\right] / 4$ which can be considerably smaller at low track momentum.

Actually, the finite size sample corrections (see Section A2) may be accounted for. Concerning the uncorrelated part, this would amount to $1 / n^{2}$ corrections and can be neglected ; the correlated part gives however a $1 / n$ contribution which corrects the term $A / 6 n$ in Rel. (B) . 9) by a factor of 4 . Therefore, an improved expression for $\sigma_{a}^{2}$ taking into account all $1 / n$ corrections is :

$$
\left.\sigma_{a}^{2}\right|_{n}=\frac{1}{4}\left[<[\delta \theta]^{2}>+\frac{1}{3} A+\frac{2}{3 n} A\right]
$$

It is also interesting to compare Rels. (B . 9) and (B . 10) with the first Rel. (B . 2 ). Indeed, one clearly sees that the variance for $a$ is smaller than the variance at mid path inside the quartz as soon as the number of photons is greater than 1 (large $n$ limit) or 4 (finite $n$ corrected). Then, in all practical applications, Rels. (B . 2 ) do not reflect the sharing of the variance between correlated and uncorrelated parts and leads to an overestimate of the multiple scattering contribution to the center errors.

\section{B3 The Full Charged Track Error Problem}

We have just treated (as a one-dimensional problem) the determination of the error on the the $a$ coordinate of the circle center, taking into account the number $n$ of emitted photons. We have seen that the uncorrelated part of its variance decreases as $1 / n$ while the correlated part is unaffected. However, our actual problem is two-dimensional and because of correlations terms like $<\delta a_{i} \delta b_{j}>$, it is not equivalent to the conjunction of two one-dimensional problems.

In order to complete the treatment, let us display the following identity for the inverse $V^{-1}$ of a symmetric matrix $V$ of rank and dimension $n$ :

$$
V^{-1}=\left(\begin{array}{cc}
S_{1} & \widetilde{C} \\
C & S_{2}
\end{array}\right)^{-1}=\left(\begin{array}{ll}
\left(S_{1}-\widetilde{C} S_{2}^{-1} C\right)^{-1} & -\left(S_{1}-\widetilde{C} S_{2}^{-1} C\right)^{-1} \widetilde{C} S_{2}^{-1} \\
-S_{2}^{-1} C\left(S_{1}-\widetilde{C} S_{2}^{-1} C\right)^{-1} & S_{2}^{-1}+S_{2}^{-1} C\left(S_{1}-\widetilde{C} S_{2}^{-1} C\right)^{-1} \widetilde{C} S_{2}^{-1}
\end{array}\right)
$$

where the submatrices $S_{1}$ and $S_{2}$ are square matrices of dimensions resp. $k(<n)$ and $n-k$, while $C$ and $\widetilde{C}$ can be square or rectangular. This identity allows to invert easily a large and structurally complicated matrix, when submatrices have peculiar forms, easy to invert (like Rel. (B.8), for instance).

Let us apply this relation to our problem. We have, by construction, $S_{1 i j}=<\delta a_{i} \delta a_{j}>$, $S_{2 i j}=<\delta b_{i} \delta b_{j}>$ and $C_{i j}=<\delta a_{i} \delta b_{j}>$, where the error functions are given by expressions like Rel. (B. 1) with appropriate path lengths. It is easy to compute these (sub-)matrices : 


$$
\left\{\begin{array}{lll}
S_{1}=B_{\theta} E+\frac{1}{24} A I & , & B_{\theta}=\frac{1}{4}\left[<[\delta \theta]^{2}>+\frac{1}{3} A\right] \\
S_{2}=B_{\phi} E+\frac{1}{24} A I & , & B_{\phi}=\frac{1}{4}\left[\sin ^{2} \theta<[\delta \phi]^{2}>+\frac{1}{3} A\right] \\
C=B_{\theta \phi} E & , & B_{\theta \phi}=\frac{1}{4} \sin \theta<[\delta \theta \delta \phi]>
\end{array}\right.
$$

where the rank 1 matrix $E$ has been already defined and still $A=c^{2} L / X_{0}$. We clearly have $C=\widetilde{C}$ and all submatrices here are $n \times n$. Rel. (B . 11) is useful in our case because these three submatrices have each a special form.

We can now define two sets of relations analogous to (B.3) for the $a_{i}$ and $b_{i}$ introducing this way $a_{0}$ and $b_{0}$, and the vector of dimension $2 n:\left(\cdots, a g_{i}-a_{i}, \cdots, b g_{j}-b_{j}, \cdots\right)$. Then we can define a function $\chi_{c}^{2}=F(a, b)$ in a way analogous to Rel. (B.3), using this vector and the matrix of Rel. (B. 11). Doing as previously, it is easy to find that the solution which minimizes $F(a, b)$ is a couple of random variables $(a, b)$ of expectation values $\left(a_{0}, b_{0}\right)$ (to be fit) with an inverse of covariance matrix defined by the sum of the elements of each of the four submatrices in Rel. (B . 11). These sums can be easily computed knowing the submatrices $S_{1}, S_{2}$ and $C$ (Rels. (B . 12)), by means of Rels. (B . 11) and (B.8).

After tedious algebra, it follows from there that the center fixing term can be written :

$$
\chi_{C}^{2}=\left(a_{0}, b_{0}\right)\left(\begin{array}{cc}
\frac{1}{4}\left[<[\delta \theta]^{2}>+\frac{1}{3} A+\frac{1}{6 n} A\right] & \frac{1}{4} \sin \theta<\delta \theta \delta \phi> \\
\frac{1}{4} \sin \theta<\delta \theta \delta \phi> & \frac{1}{4}\left[\sin ^{2} \theta<[\delta \phi]^{2}>+\frac{1}{3} A+\frac{1}{6 n} A\right]
\end{array}\right)^{-1}\left(\begin{array}{c}
a_{0} \\
b_{0}
\end{array}\right)
$$

In writing this expression, we have used $a_{0}$ and $b_{0}$ instead of $a_{0}-a_{\text {measured }}$ and $b_{0}-b_{\text {measured }}$, taking into account that the corresponding measured values are zero by definition. This relation defines the error covariance matrix of the charged track direction associated with $n$ detected photons ; it differs from the matrix $\Sigma_{0}$ by its taking into account the multiple scattering undergone by the charged track inside the radiator. It can simply be written $\Sigma=\Sigma_{0}+[A / 3+$ $A /(6 n)] I$.

One can apply the finite size sample corrections found in Section A2, as we did in the previous subsection. This amounts to change the expression for $\Sigma$ to $\Sigma=\Sigma_{0}+[A / 3+2 A /(3 n)] I$.

Finally, it should be noted that the $a_{0} b_{0}$ covariance term is not affected by effects due to multiple scattering; this could have been inferred from Rels. (B.1) (and explains the covariance term in Rels. (B.2), as the expectation value $\left\langle\varepsilon_{1}(u) \varepsilon_{2}(v)>\right.$ is zero for any values of $u$ and $v$ ). From now on, we name for clarity the parameters to be fit $a$ and $b$ instead of $a_{0}$ and $b_{0}$.

It should be noted, however, that multiple scattering effects imply that there are as many centers (charged track directions) as photons. Therefore, any fit procedure can only provide a determination of the mean center coordinates for each track considered, as an approximation of the actual center value at the DIRC bar entrance. 


\section{B4 The Minimization Function and the Circle Parameters}

Given a set of points known each with some error, and assuming they should be on a circle arc, the problem we state here is to define a function $F(a, b, R)$, the minimum of which providing the circle parameters. An usual approach is actually to choose as function $F$, a $\chi^{2}$. Denoting by $R$ the circle radius and by $(a, b)$ the center coordinates, the function is :

$$
\chi_{n}^{2}=\sum_{i, j=1, n}\left(d_{i}-R g_{i}\right) V_{i j}^{-1}\left(d_{j}-R g_{j}\right)
$$

where $d_{i}=\sqrt{\left(x_{i}-a\right)^{2}+\left(y_{i}-b\right)^{2}}$ is the distance of each point $\left(x_{i}, y_{i}\right)$ to the fit center, and $g_{i}=1$ defines a constant vector $g$ introduced only in order to have a correct matching of repeated indices. A priori, the covariance matrix is defined by $V_{i j}=<\delta d_{i} \delta d_{j}>$. Usually, the error functions $\delta d_{i}$ are obtained by differentiating the expression for $d_{i}$ :

$$
\delta d_{i}=\frac{\left(x_{i}-a\right) \delta x_{i}+\left(y_{i}-b\right) \delta y_{i}}{d_{i}}
$$

where $\delta x_{i}$ and $\delta y_{i}$ are the errors functions affecting the photon measurements $x_{i}$ and $y_{i}$.

In our case, the points are spread onto a small arc and/or the relative size of the errors compared with the circle radius is large ; then, one has to introduce additional information for reasons already quoted at several places in the body of this paper. The most obvious additional information which is available in our case refers to the circle center.

We have as a priori information the measurement provided by the tracking device located in front of the DIRC bar entrance ; this is summarized by a central value and a covariance error matrix (referred to anywhere above as $\Sigma_{0}$ ). Obviously, this defines a distribution (normal, assuming we are lucky) but not the actual location of the center indeed associated with the track under consideration.

The question is now : how to introduce the approximate knowledge $(0,0)$ of the given charged track direction and keep anyway its actual center coordinates $(a, b)$ to be fit? In the previous case (no charged track information), the measured quantities could be written :

$$
x_{i}=x_{i}^{0}+a+\delta x_{i}, \quad y_{i}=y_{i}^{0}+b+\delta y_{i}
$$

where $x_{i}^{0}=R \cos \varphi_{i}$ and $y_{i}^{0}=R \sin \varphi_{i}$. Here $R$ is the true radius, $\varphi_{i}$ is the true azimuth on the circle and $(a, b)$ the true center. Then the true value for the $x_{i}$ and $y_{i}$ are respectively $x_{i}^{0}+a$ and $y_{i}^{0}+b$. Fixing the center at the "measured" value $\left(a_{i}, b_{i}\right)$ (actually $(0,0)$ ), turns out to rewrite these equations :

$$
x_{i}=x_{i}^{0}+a_{i}+\delta x_{i} \quad, \quad y_{i}=y_{i}^{0}+b_{i}+\delta y_{i}
$$

However, for a given well defined track, we can write :

$$
a_{i}=a+\delta a_{i} \quad, \quad b_{i}=b+\delta b_{i} \quad, \quad \forall i=1, \cdots n
$$

where $\delta a_{i}$ and $\delta b_{i}$ are the error functions which take into account the errors at the entrance of the DIRC bar and the multiple scattering undergone by the charged track up to the point where it emits photon $i$. In this way $(a, b)$ is the actual center when it exists (no multiple scattering), otherwise it can be formally defined as the mean value of the quantity corresponding to $G$ in 
Rel. (A . 13), which is then non-zero on a track by track basis. Then Eqs. (B . 17) can be rewritten :

$$
x_{i}=x_{i}^{0}+a+\delta a_{i}+\delta x_{i}, \quad y_{i}=y_{i}^{0}+b+\delta b_{i}+\delta y_{i}
$$

The difference between the case when the center is left free and when it is constrained is transfered to the error functions which become $\delta a_{i}+\delta x_{i}$ and $\delta b_{i}+\delta y_{i}$ instead of respectively $\delta x_{i}$ and $\delta y_{i}$. Conceptually, the difference comes from what is submitted to fit in both cases. In the former case (free center), the measured quantitites submitted to fit are the measured points $\left(x_{i}, y_{i}\right)$, while in the latter case (constrained center), the quantitites submitted to fit are actually $\left(x_{i}-a_{\text {measurement }}, y_{i}-b_{\text {measurement }}\right)$. In the former case, the center $(a, b)$ is fully fit, in the latter case one fits the departure of the actual center from the measured point $(0,0)$.

Then, Rel. (B.15) is still valid but should be rewritten :

$$
\delta d_{i}=\frac{\left(x_{i}-a\right)\left(\delta x_{i}+\delta a_{i}\right)+\left(y_{i}-b\right)\left(\delta y_{i}+\delta b_{i}\right)}{d_{i}}
$$

in order to keep $\delta x_{i}$ and $\delta y_{i}$ their original meaning (errors due to the measurement of the photon direction without reference to the charged track). If, moreover, we choose the origin in the plane in order that it coincides with the image of the track direction provided by the tracking device, Rel. (B.20) can be approximated by :

$$
\delta d_{i}=\frac{x_{i}\left(\delta x_{i}+\delta a_{i}\right)+y_{i}\left(\delta y_{i}+\delta b_{i}\right)}{d_{i}^{\prime}}
$$

where $d_{i}^{\prime}$ in the denominator is $d_{i}^{\prime}=\sqrt{x_{i}^{2}+y_{i}^{2}} . \delta d_{i}$ in Rels. (B.20) and (B.15) differ only at first order and only by the differentials for $\delta a_{i}$ and $\delta b_{i}$.

Therefore, the quantity $\chi_{n}^{2}$ (Rel. (B . 14) ) can be used with $V_{i j}=<\delta d_{i} \delta d_{j}>$, where the errors functions are given by Rel. (B.21). As they depend on the path length followed up to the emission of each photon, this expression has to be approximated by its mean value $V_{i j}=<<\delta d_{i} \delta d_{j}>>_{u_{i} u_{j}}$ easily computable using all information given above.

In this way, we are in position to define $\chi_{n}^{2}$ as a function of $(a, b)$ and $R$. It remains to account for forcing the coordinates $(a, b)$ to remain in the neighborhood of the measured point $(0,0)$; this is achieved 22 by defining the following $\chi^{2}$ :

$$
\chi^{2}=\chi_{n}^{2}+\chi_{C}^{2}
$$

using Rels. (B . 14) and (B.13), where the influence of multiple scattering is already taken into account, including correlations. While $\chi_{n}^{2}$ is $n-3$ degrees of freedom, the $\chi^{2}$ just defined is $n-1$ degrees of freedom.

One could ask oneself about the influence of the additional term $\chi_{C}^{2}$ when minimising, if the circle arc happens to be large enough that such a term is actually useless. We have checked numerically this case using trivial simulations, where the populated arc length and the number of "measured" points could be varied at will. We have found that for large circle arcs (about

\footnotetext{
${ }^{22}$ One may ask oneself whether Rel. (B . 22) actually exhausts the problem. Indeed, one may be tempted to introduce in the $\chi^{2}$ to be minimized, terms coupling $a$ and $b$ with the $d_{i}-R g_{i}$; this is not studied here. Anyway, such additional terms would surely degrade the algorithm speed. From the results already at hand, one may conclude that their effect is small for track momenta above $500 \mathrm{MeV} / \mathrm{c}$.
} 
$180^{\circ}$ or more) the additional term $\chi_{C}^{2}$ did not prevent to get the same solution as when it is removed, with completely negligible fluctuations.

Another possibility could be considered. This turns to give up fitting the center, accepting the measured value $(0,0)$ as optimum. Using the notation $d_{i}^{\prime} \equiv d_{i}(a=0, b=0)$, this turns to estimate the radius by :

$$
R=\sigma_{R}^{2} \sum_{i j} g_{i} V_{i j}^{-1} d_{j}^{\prime} \quad, \quad \frac{1}{\sigma_{R}^{2}}=\sum_{i j} g_{i} V_{i j}^{-1} g_{j}
$$

This gives results close in quality to minimising Eq. (B . 22), if the fixed center $(a, b)=(0,0)$ is correctly measured. If however, there is any bias in this estimate, it may become much worser. Indeed, in case when the measured center is slightly biased, minimising Eq. (B. 22) is safe as one recovers the correct center location, even starting from a wrong center ; instead, using Eq. (B . 23), the estimate of the radius suffers piling up effects which summarize in sensible effects. For instance, assuming $2.8 \mathrm{mr}$ systematic error on the charged track direction (known with statistical accuracy $2 \mathrm{mr}$ rms), the radius pull gets a rms of 1.12 instead of 0.98 , under the same conditions, for the procedure we recommand. This difference of behaviour degrades in presence of background (1.4 compared to 1.2) or if the statistical accuracy on the charged track direction worsens.

\section{B5 Correlations among Photons in the Equatorial Plane}

The radius $\tan \theta_{C} / 2$ of the circle is approximated by each of the photon distance to the

common center $\left(d_{i}^{\prime}=\sqrt{x_{i}^{2}+y_{i}^{2}}\right)$. Each such estimate is affected by an error function which is given by Rel. (B.21), with the measured center set at the origin in the equatorial plane. From expressions given above, we can then deduce :

$$
\left\{\begin{aligned}
<\left[\delta d_{i}\right]^{2}>= & \frac{1}{d_{i}^{\prime 2}}\left[x_{i}^{2}\left[<\left[\delta x_{i}\right]^{2}>+\frac{1}{4}<[\delta \theta]^{2}>\right]+y_{i}^{2}\left[<\left[\delta y_{i}\right]^{2}>+\frac{1}{4} \sin ^{2} \theta<[\delta \phi]^{2}>\right]+\right. \\
& \left.2 x_{i} y_{i}\left[<\delta x_{i} \delta y_{i}>+\frac{1}{4} \sin \theta<\delta \theta \delta \phi>\right]\right]+\frac{1}{8} c^{2} \frac{L}{X_{0}} \\
<\delta d_{i} \delta d_{j}>= & \frac{1}{4 d_{i}^{\prime} d_{j}^{\prime}}\left[x_{i} x_{j}<[\delta \theta]^{2}>+y_{i} y_{j} \sin ^{2} \theta<[\delta \phi]^{2}>+\right. \\
& \left.\left(x_{i} y_{j}+x_{j} y_{i}\right) \sin \theta<\delta \theta \delta \phi>\right]+\frac{\left(x_{i} x_{j}+y_{i} y_{j}\right)}{d_{i}^{\prime} d_{j}^{\prime}} \frac{1}{12} c^{2} \frac{L}{X_{0}}
\end{aligned}\right.
$$

where we have assumed that the measurements $(x$ and $y$ ) for photons $i$ and $j$ are statistically independent for ease of reading.

These relations give the expression for the elements of the error covariance matrix which enter the fit procedure described in the body of the text and just above. The variance for each estimate of the circle radius $\left(d_{i}^{\prime}\right)$ depends on the photon errors, the track direction errors and the multiple scattering it undergoes ; the covariance term exhibits an interesting feature : up to the fact that the metric along $\vec{v}$ and $\vec{w}$ are different, one sees a surprising correlation pattern. 
Qualitatively, correlations are the strongest (and positive) for photons close to each other in azimuth, correlations are the strongest (and negative) for pairs of photons opposite in azimuth, while there is no correlation for photon pairs having azimuthal distance of $\pi / 2$.

\section{B6 Multiple Scattering Effects in the General Case}

When the arc to be fit is large enough (possibly $2 \pi$ radians), fixing the circle center becomes irrelevant. In this case, a question remains about the influence of multiple scattering on the error definition and the fit procedure.

For each photon, $d_{i}=\sqrt{\left(x_{i}-a_{i}\right)^{2}+\left(y_{i}-b_{i}\right)^{2}}$ remains the basic quantity which enters the fit procedure. Whatever is the way to express the problem, we have as free parameter the charged track direction and as "data" the angular distance of this direction with photon directions. As noted above, when multiple scattering is active, the direction of the charged track varies from photon to photon with theoretically known statistical fluctuations. Therefore the estimate of the radius provided by each photon inherits the fluctuations of the charged track. Stated otherwise, the error due to multiple scattering can either be treated separately (as we did) or included in the error function of the measurement $\left(x_{i}, y_{i}\right)$, together with the other contributions (geometrical errors, chromaticity error). This means that Rel. (B. 21) is still relevant. Therefore, when there is no center fixing term, it is equivalent to consider that the error function on $\left(x_{i}, y_{i}\right)$ is $\left(\delta x_{i}+\delta a_{i}, \delta y_{i}+\delta b_{i}\right)$, where the second term of each component is reduced to only the multiple scattering contribution.

In this case, Rels. (B.24) become :

$$
\left\{\begin{array}{l}
<\left[\delta d_{i}\right]^{2}>=\frac{1}{d_{i}^{\prime 2}}\left[x_{i}^{2}<\left[\delta x_{i}\right]^{2}>+y_{i}^{2}<\left[\delta y_{i}\right]^{2}>+2 x_{i} y_{i}<\delta x_{i} \delta y_{i}>\right]+\frac{1}{8} c^{2} \frac{L}{X_{0}} \\
<\delta d_{i} \delta d_{j}>=\frac{\left(x_{i} y_{j}+x_{j} y_{i}\right)}{d_{i}^{\prime} d_{j}^{\prime}} \frac{1}{12} c^{2} \frac{L}{X_{0}}
\end{array}\right.
$$

This shows that correlations among photons always exist, only due however to the properties of multiple scattering. Therefore, for low momentum tracks, when the multiple scattering is dominant, correlations among photons can never be ignored in any reconstruction procedure for devices like the DIRC. This is clearly independent of the representation chosen for the data (here the stereographic projection) ; it only relies on the fact that the measured quantities are angles between photons and a single charged track direction which changes in a correlated way from one photon to the other. 


\section{Acknowledgements}

We thanks our colleagues of the BaBar DIRC group for the interest they manifested in the

successive steps of this work. We also warmly acknowledge J. Chauveau for important remarks and comments.

\section{References}

[1] T. Ypsilantis and J. Séguinot, Nucl. Inst. and Meth. A343 (1994) 30.

[2] J. Séguinot et al., Nucl. Inst. and Meth. A350 (1994) 430.

[3] P. Baillon, Nucl. Inst. and Meth. A238 (1985) 341.

[4] P.Coyle et al., Nucl. Inst. and Meth. A343 (1994) 292.

[5] R.Aleksan et al., Nucl. Inst. and Meth. A397 (1997) 261.

[6] Babar Technical Design Report, SLAC-R-95-457, March 1995.

[7] D. Hatzifotiadou et al., CERN-LAA/PI-94-17, Geneva 1994 ; T. Ypsilantis, J. Séguinot and A Zichichi, CERN-LAA/96-13, Geneva 1996.

[8] I. Adam et al., SLAC-PUB-7706, Stanford, Nov. 1997; preprint hep-ex/9712001, Dec 1 1997. Invited talk presented at the 1997 IEEE Nuclear Science Symposium and Medical Imaging Conference, Albuquerque, New Mexico, Nov 9-15 1997. J. Schwiening et al. SLACPUB-7706, Dec. 1997; preprint hep-ex/9712018, Dec 12 1997. Invited talk presented at the 5th International Workshop on B-Physics at Hadron Machines (Beauty '97), Santa Monica, California, October 13-17, 1997.

[9] LHCb Technical Proposal, CERN LHCC 98-4, LHC/P4, Geneva, Feb. 1998.

[10] G. Julia, Eléments de Géometrie Infinitésimale, Gauthier-Villars, Paris 1936.

[11] J.F. Crawford, Nucl. Inst. and Meth. 211 (1983) 223.

[12] N.I. Chernov and G..A. Osokov, Comp.. Phys. Comm. 33 (1984) 329.

[13] V. Karimäki, Nucl. Inst. and Meth. A305 (1991) 187.

[14] P.L. Rosin, Pattern Recognition Letters 14 (1993) 661

[15] P.J. Huber, Robust statistics (Wiley, 1981)

[16] Review of Particle Properties, R.M. Barnett et al., Phys. Rev. D54 (1996)1. 

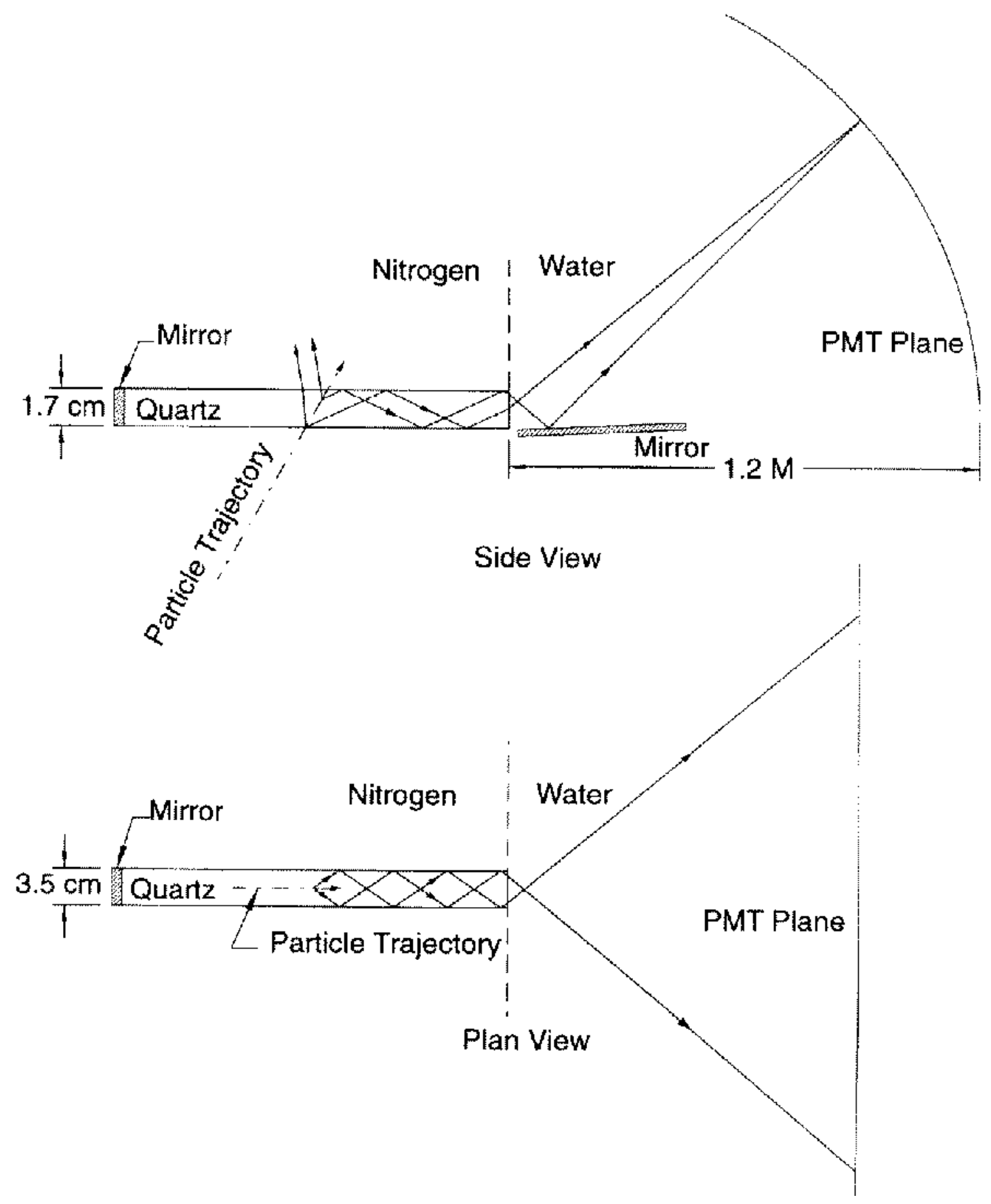

Figure 1: A schematic drawing illustrating the DIRC principle : photons produced in the quartz bar radiator are transported to the bar end because of total internal reflection. Photon angles are preserved till bar exit. 

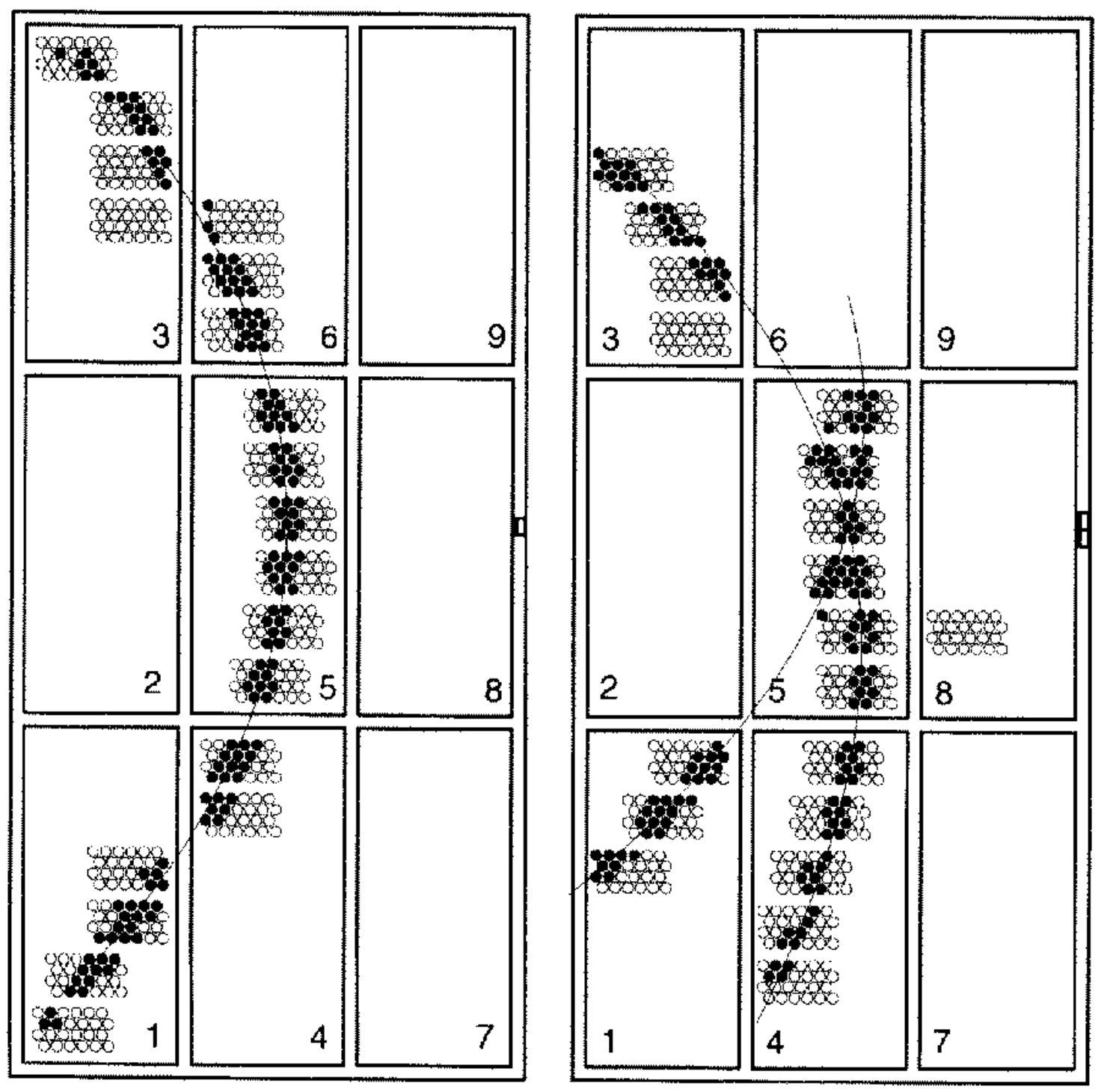

Figure 2: Display of a single track event in the DIRC prototype showing the superposition of reflections of the original Cerenkov cone. 


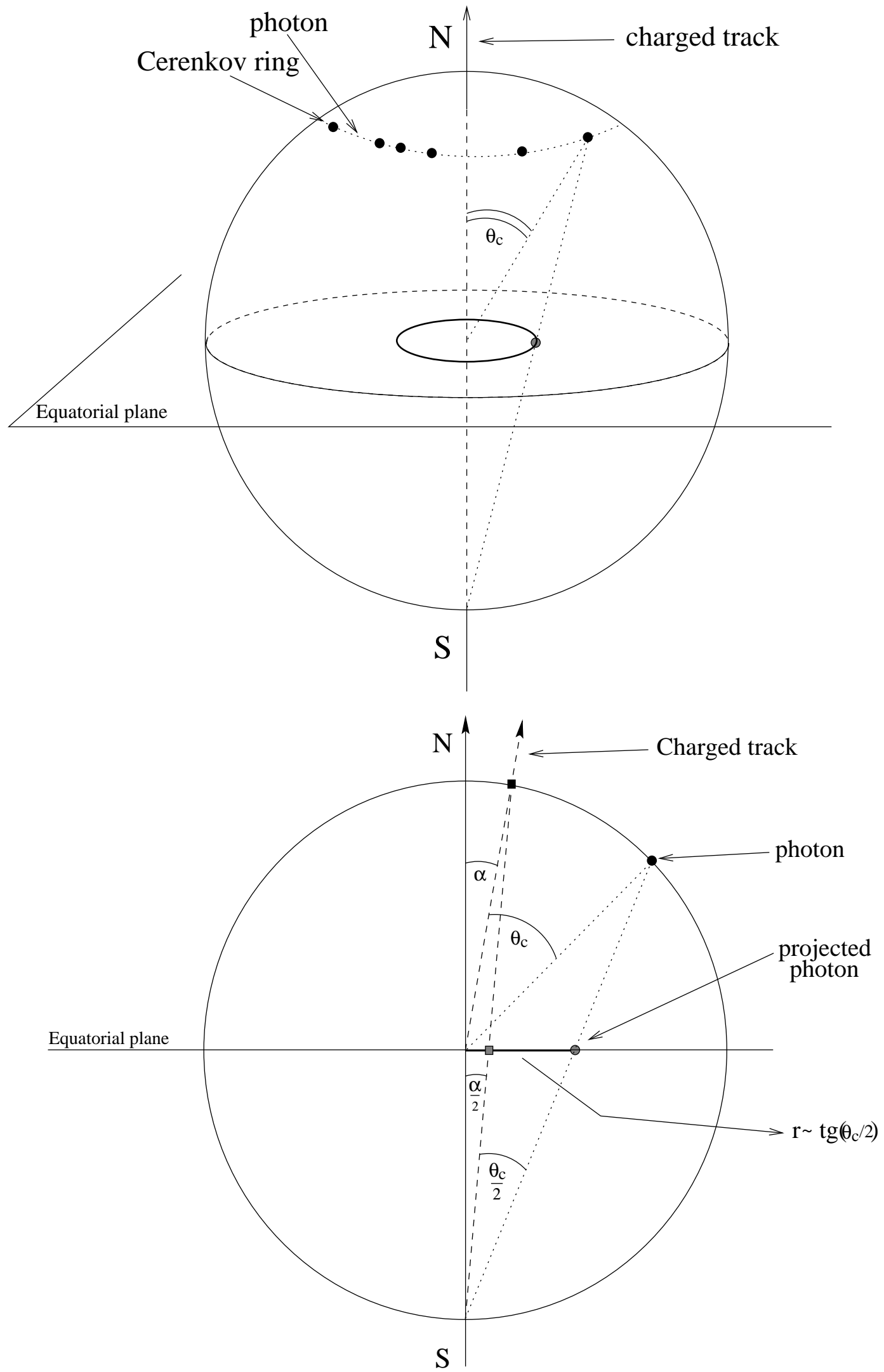

Figure 3: Stereographic projection. 


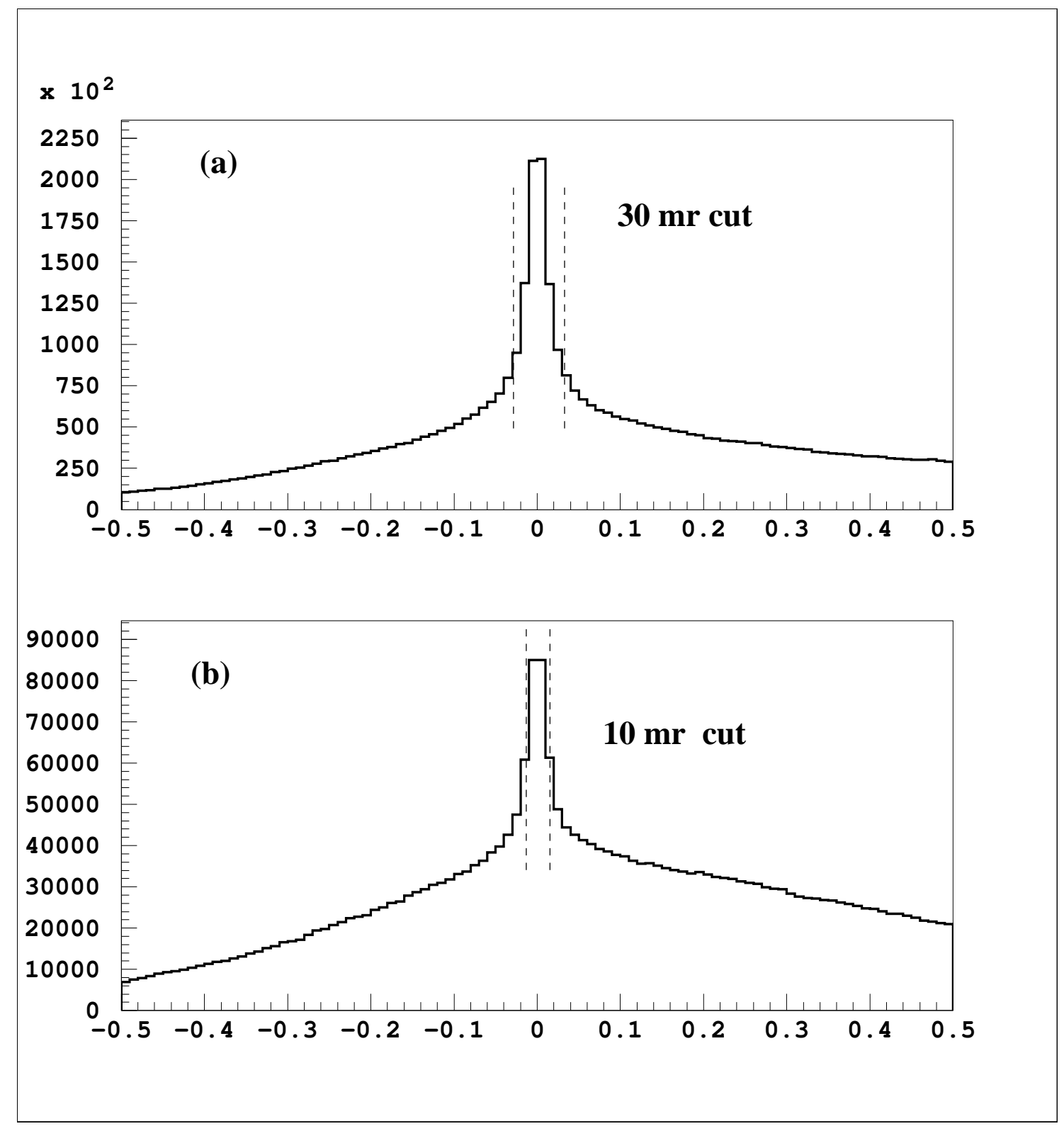

Figure 4: Distribution of $\delta \theta$ (radian units) for the mixed track sample with one additional reconstructed background track; in (a), $\delta \theta$ as measured from signal track; $\delta \theta$ in (b), as measured from background track. 


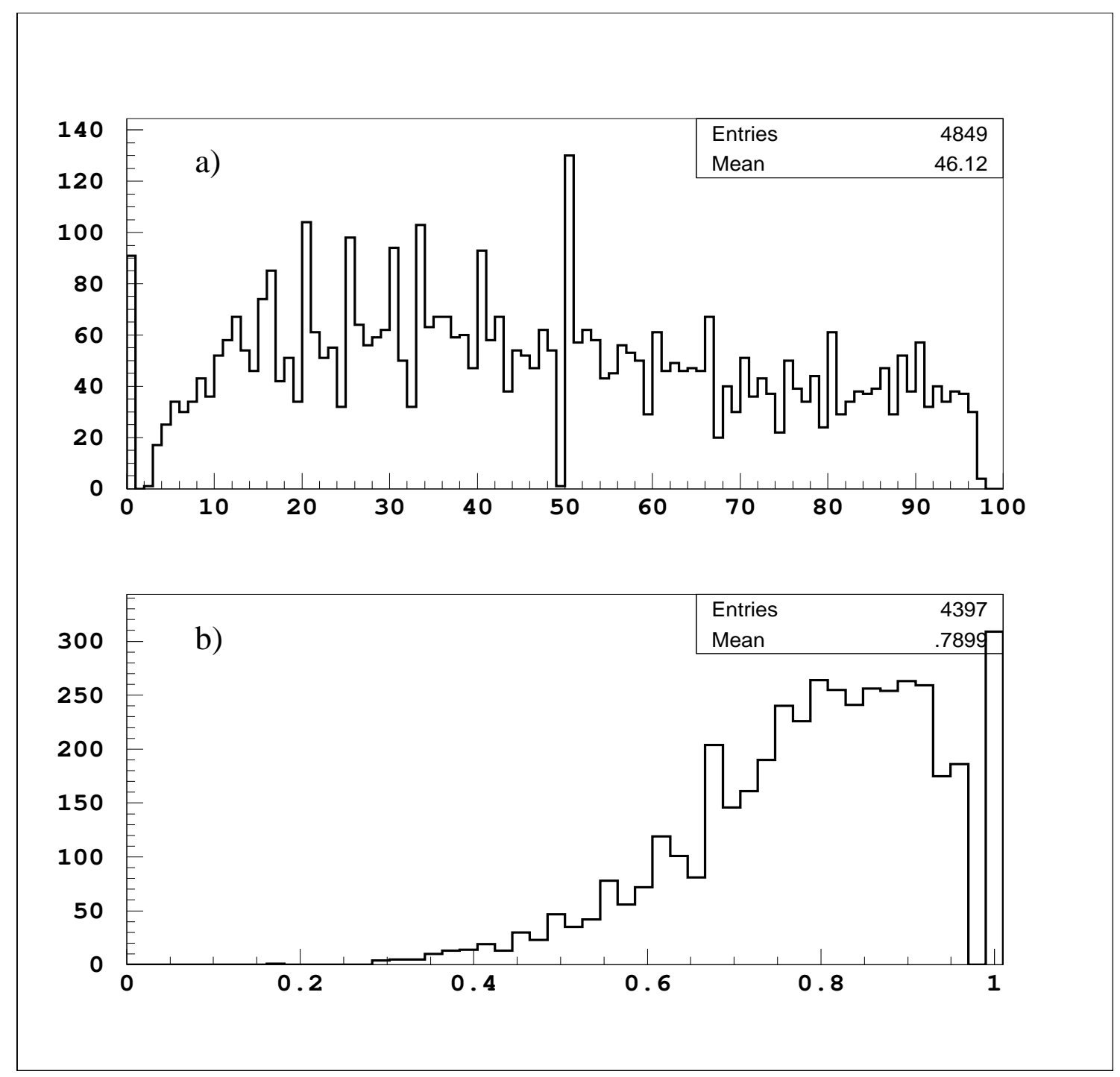

Figure 5: In a), histogram of the proportion (in percents) of unambiguous photons relative to the total number of detected photons (all are signal photons) for each track before the photon recovering step. Non accepted events appear as a peak at zero. In b), histogram of the same quantity after the recovering procedure. In both cases no flat background and no additional tracks have been added. 

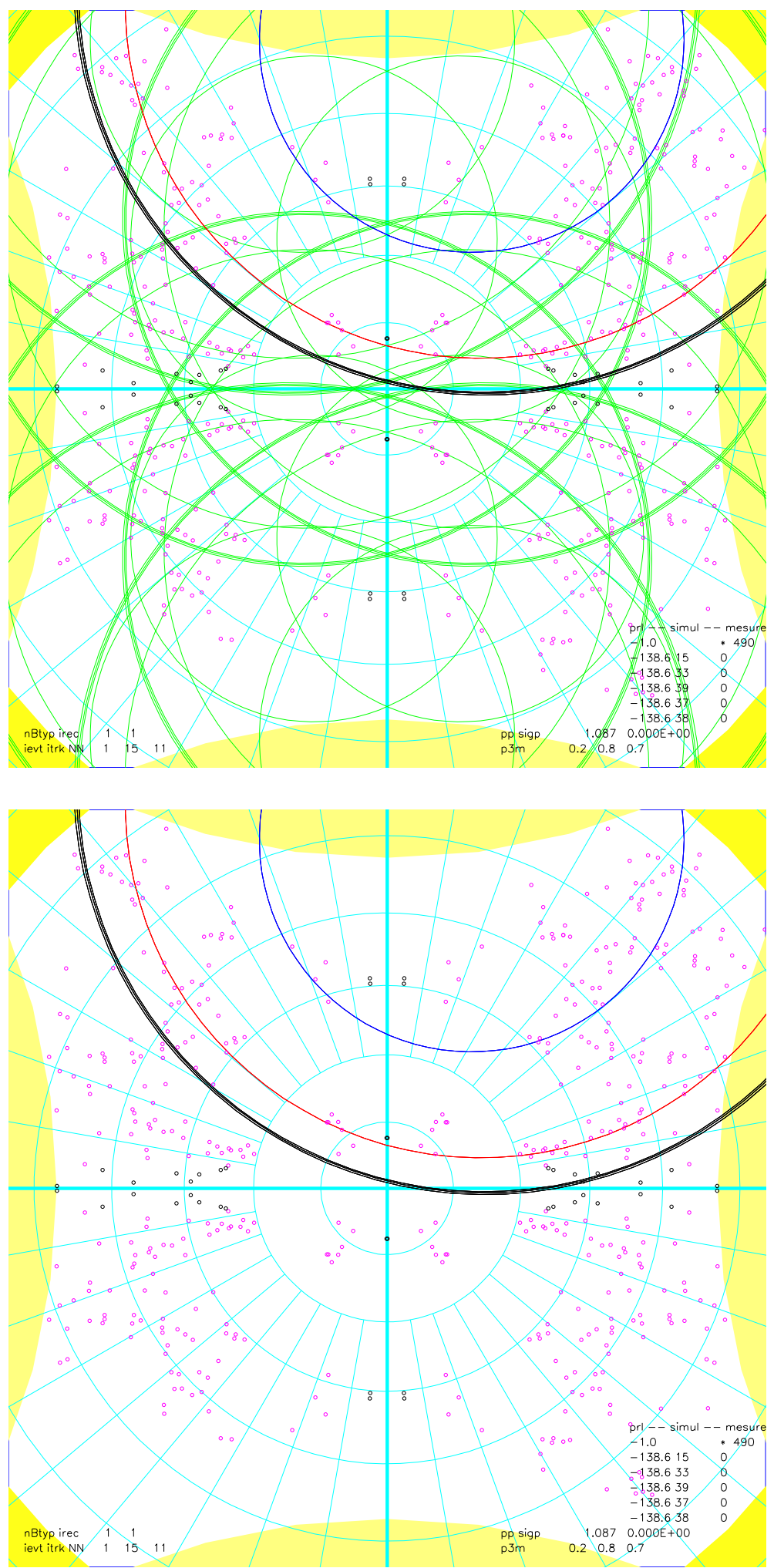

Figure 6: Display of DIRC Cerenkov (solution) photons for one kaon track produced together with a low flat background (see text). An equatorial projection wrt the bar axis is used here. Top : all photon solutions surviving the primary selection cuts are displayed with the various reflected circles ; Bottom : same view, keeping only the circles associated with the original track direction. 

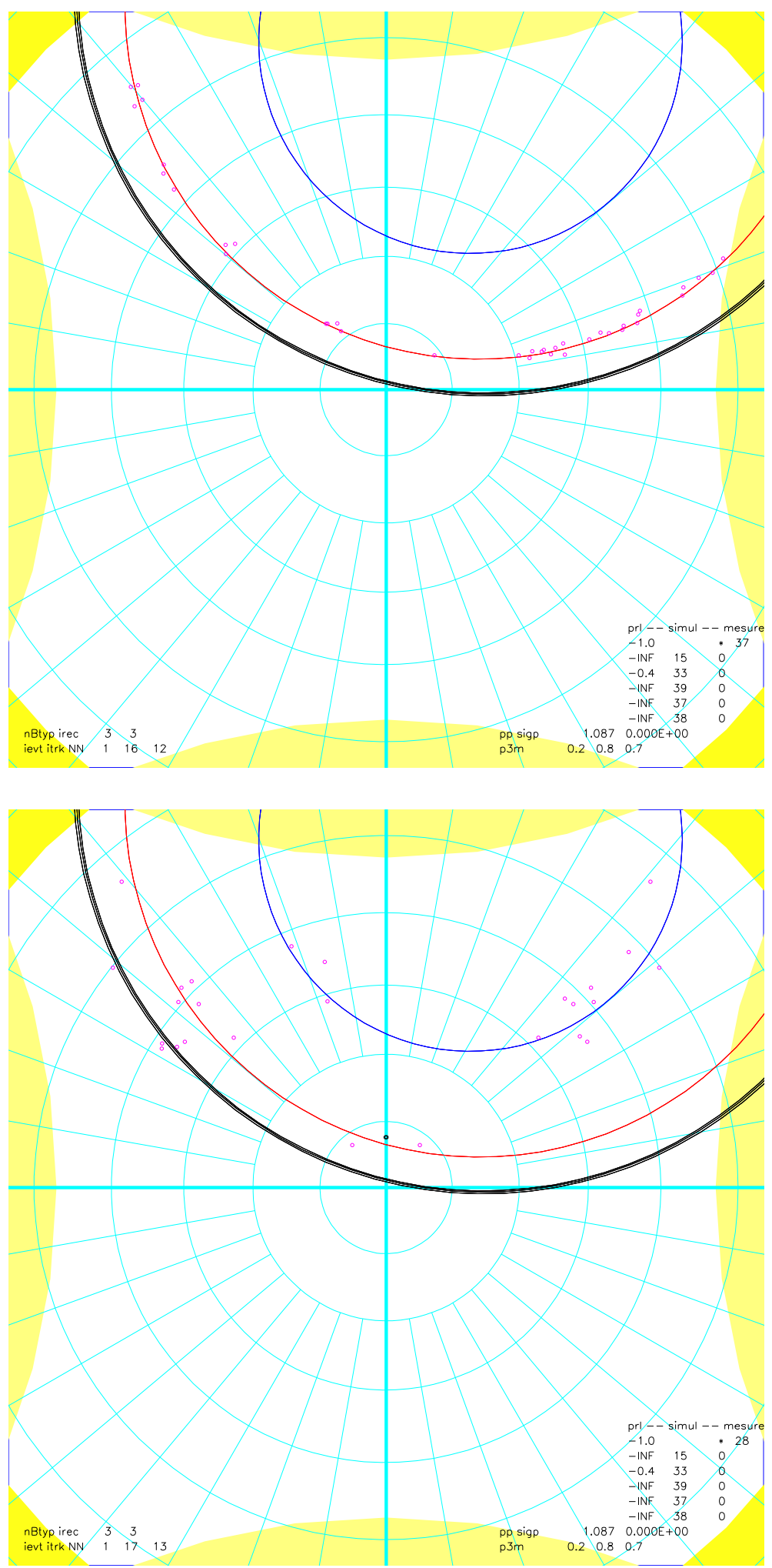

Figure 7: Display of DIRC Cerenkov (solution) photons for the event shown in Fig. 6. Top : unambiguous photon solutions found by the algorithm ; all lay along the kaon circle. Bottom : all ambiguous photon solutions to be examined by the recovering procedure : they are spread along all possible circles. 


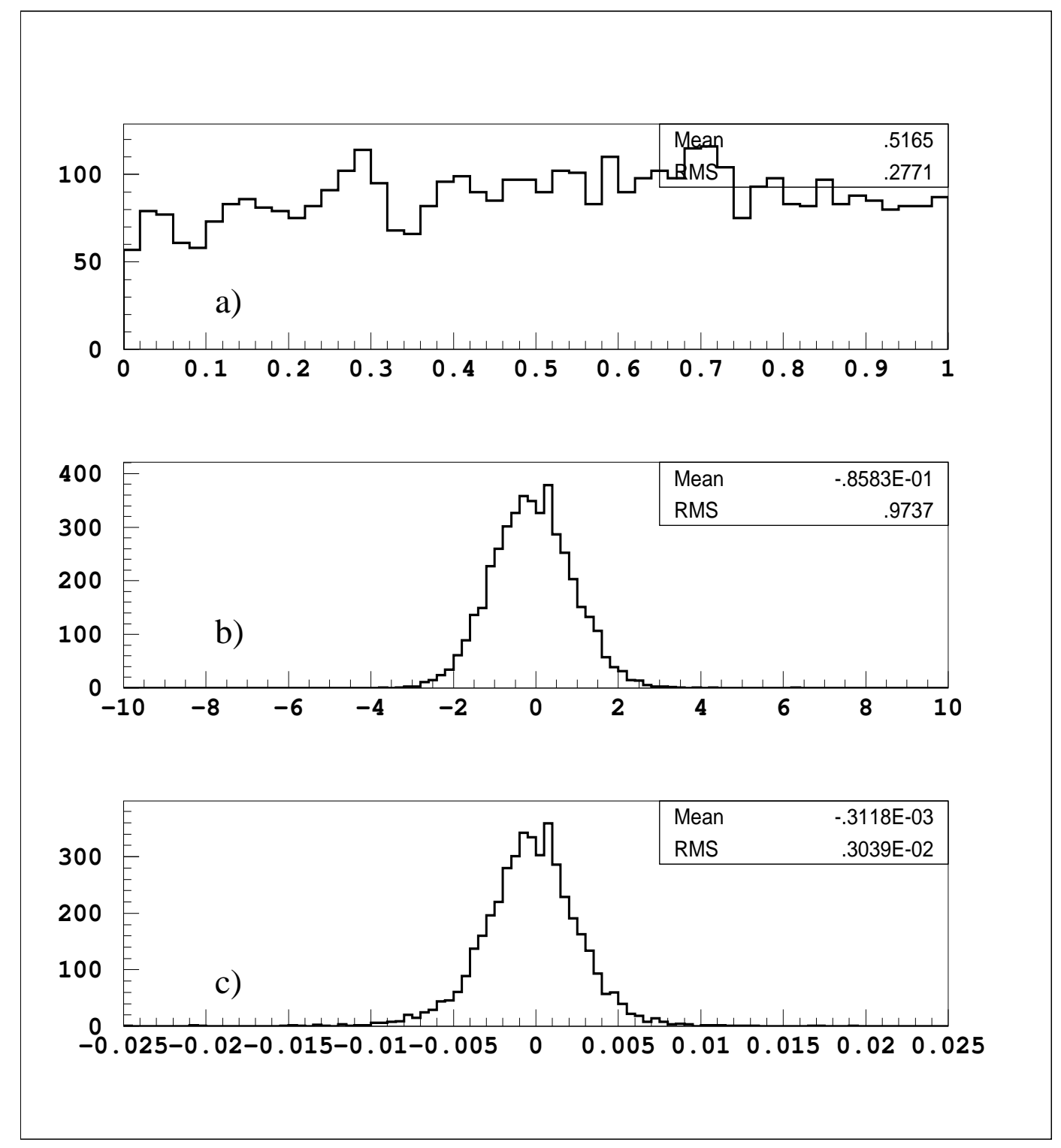

Figure 8: Distributions obtained using Monte Carlo data generated without additional noise. In a) the $\chi^{2}$ probability distribution ; in b), the reconstructed Cerenkov angle pulls ; in c), the absolute biases (radians). Notice in b) and c) the smallness of the tails. 
a)
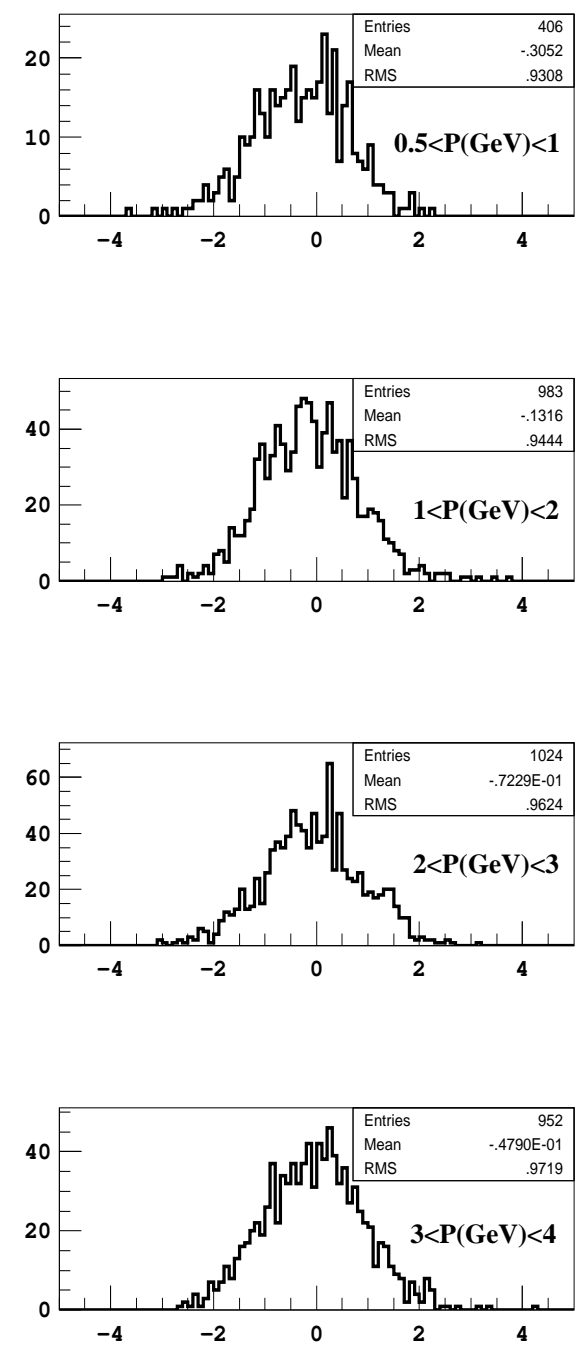

b)
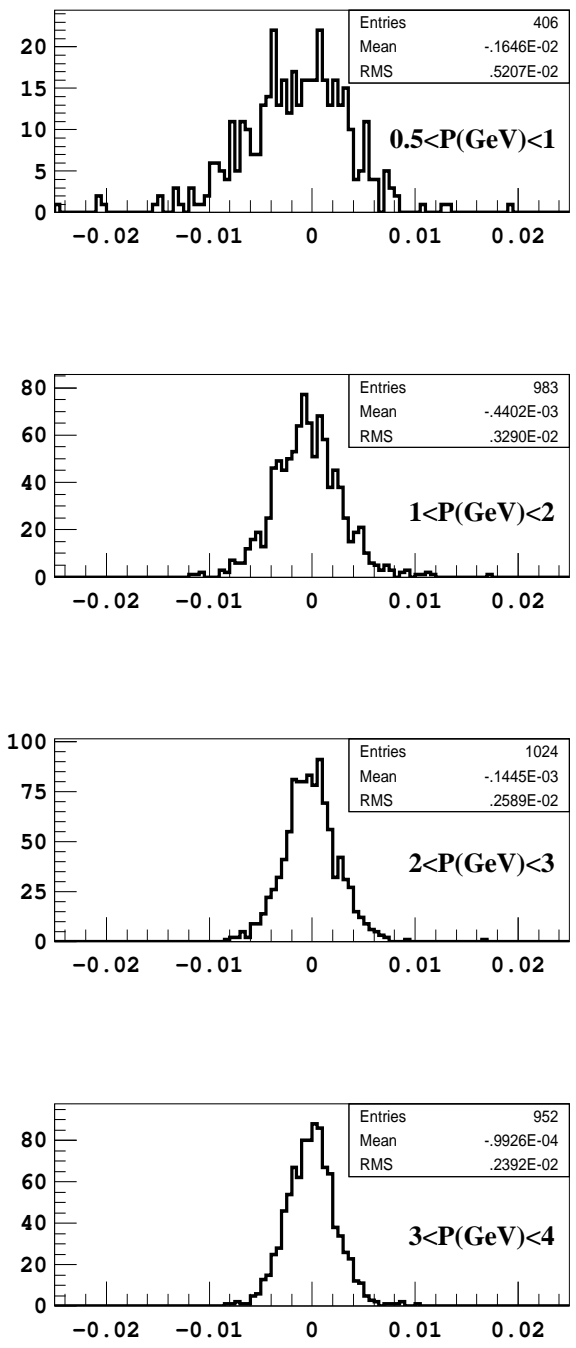

Figure 9: Estimated error behavior. In a) the estimated pull dispersion (rms) of reconstructed Cerenkov angle around the expected Cerenkov angle in various track momentum bins ; b) rms dispersion of the reconstructed Cerenkov angle (in radians) also in the same track momentum bins. Notice in a) the trend to go towards a pull of zero mean and unit rms, when going to higher and higher momenta ; this typically reflects the decreasing effect of multiple scattering. 

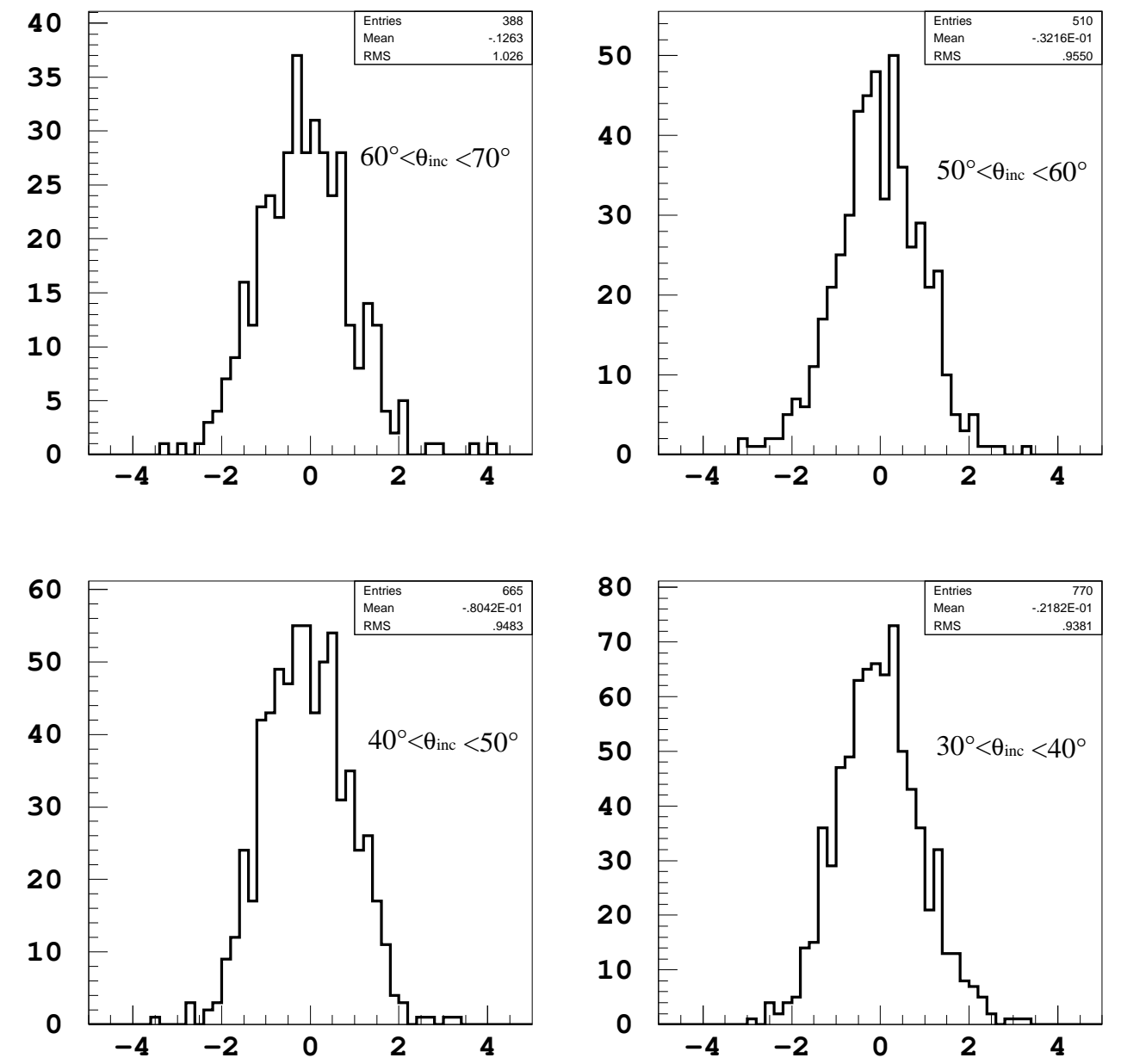

Figure 10: Reconstructed Cerenkov angle pull in a few bins of track incidence angle on the bar. The mean and rms are close to the expected values, except in the $60^{\circ}<\theta_{\text {inc }}<70^{\circ}$ bin, where the mean is slightly too large. 

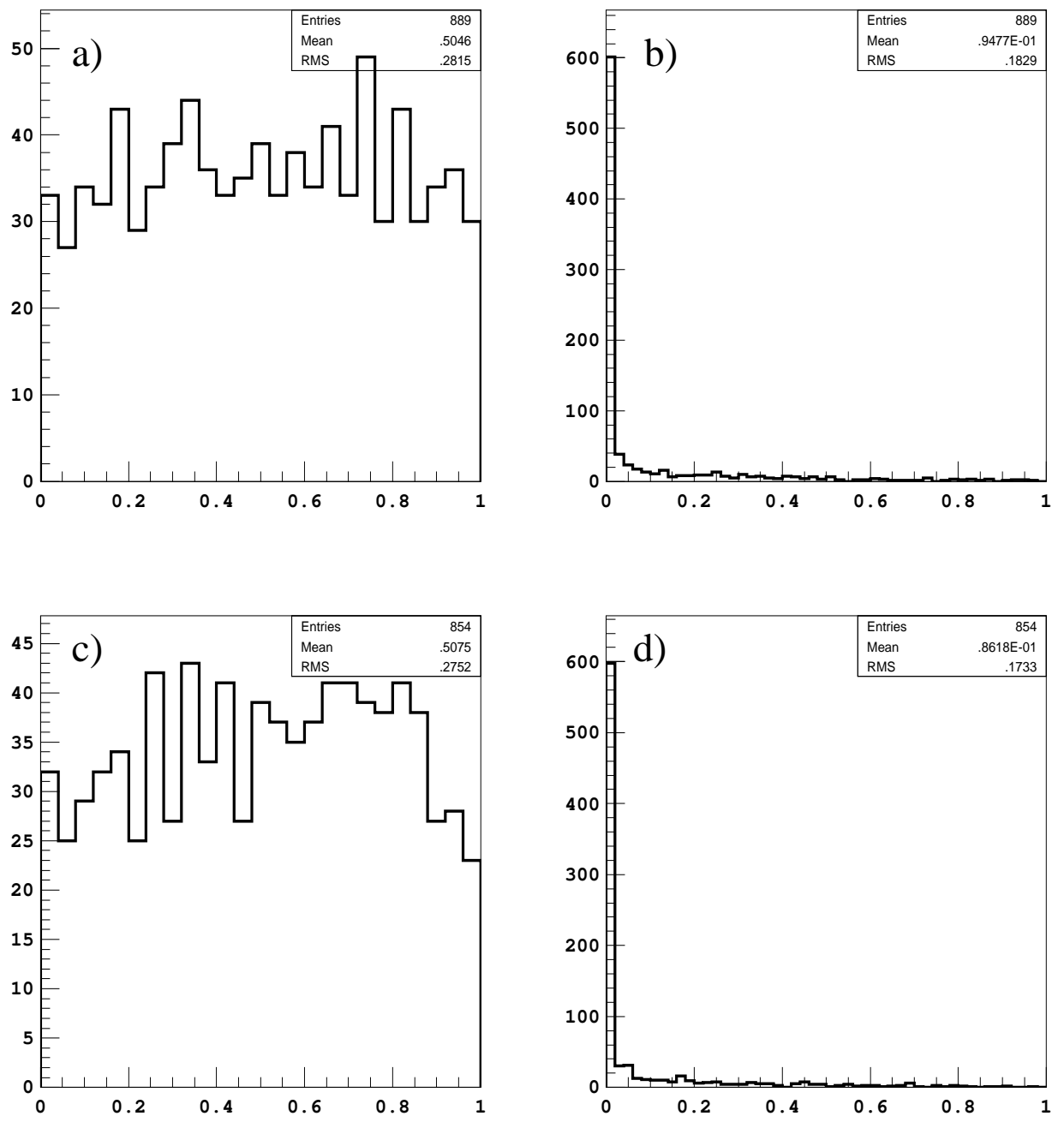

Figure 11: $\chi^{2}$ probabilities of pion and kaon identification. In a), pion hypothesis probability for true pion tracks ; in b), kaon hypothesis probability for true pion tracks ; in c), kaon hypothesis probability for true kaon tracks ; in d), pion hypothesis probability for true kaon tracks. 

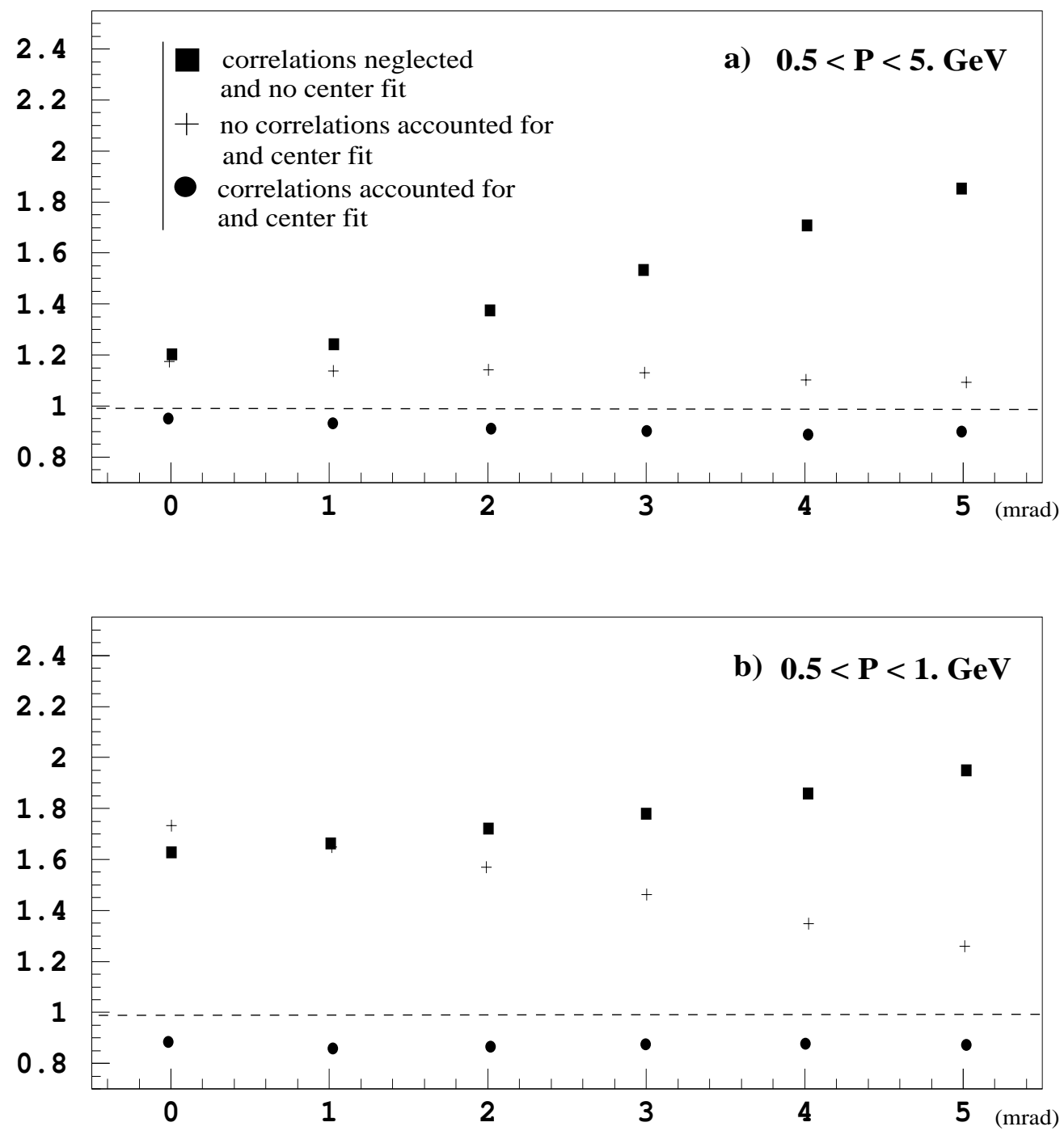

Figure 12: An example of the Cerenkov circle fit and the inter photon correlations effect when the errors on the charged track direction are changed. In a), the full track momentum range is used, whereas in b) only low momentum tracks are considered. Along the horizontal axis, the dispersion of the track polar angles in milliradian units $\left(\sigma_{\theta}=\sigma_{\phi}\right)$; along the vertical axis, the RMS dispersion of the pulls of the reconstructed Cerenkov angle. Square markers represent the pulls dispersion value when the correlations are neglected and the center is not fitted, cross markers show the same quantity when the center is fitted ; finally, round markers show the same quantity when correlations are taken into account and the center is fitted. Fitting the center allows a partial account of correlations (see text). 


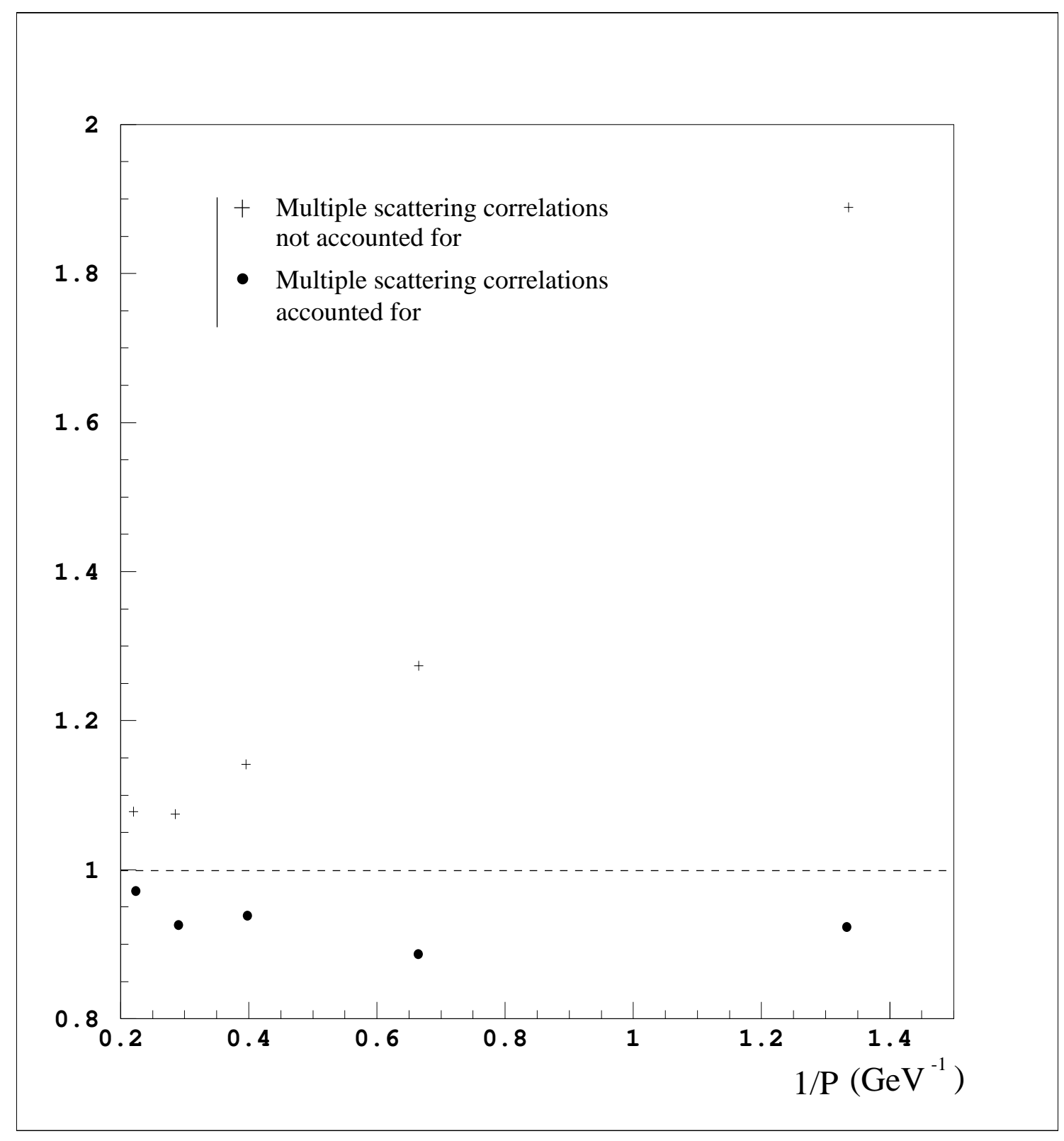

Figure 13: Effect of inter photon correlations due to multiple scattering (the dispersion of track direction measurement is set to zero). The reconstructed Cerenkov angle pull rms is plotted as a function of the inverse track momentum : cross markers represent the pulls dispersion when no correlations are accounted for ; the round markers show the stability of the pulls when the correlations are correctly taken into account. 

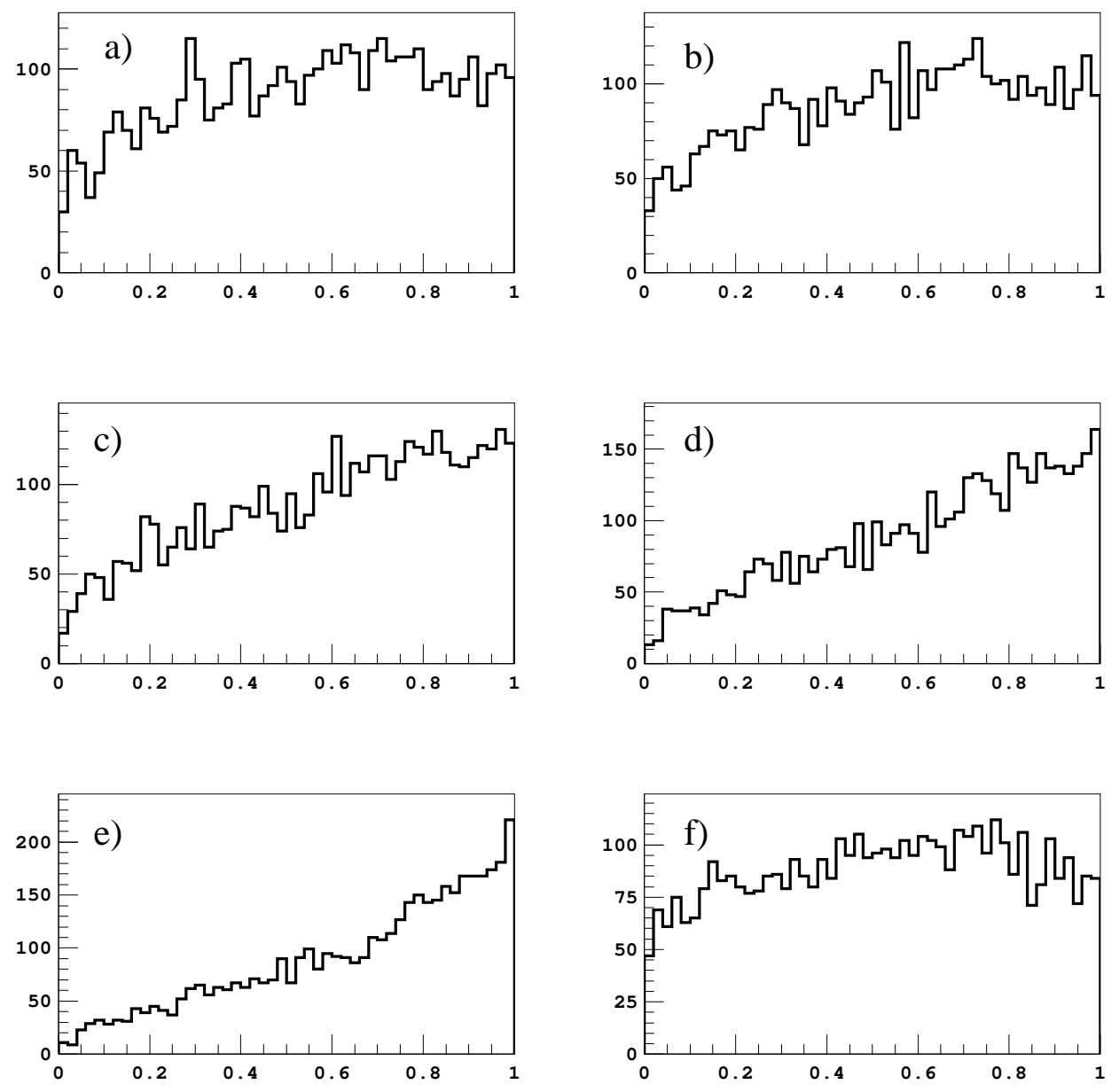

Figure 14: Influence of the errors $(\delta \theta, \delta \phi)$ on the incoming track direction on the $\chi^{2}$ probability distribution when the inter photon correlations are not taken into account. In a) $\delta \theta=\delta \phi=0$ $\mathrm{mr}$; in b), $\delta \theta=\delta \phi=1 \mathrm{mr}$; in c), $\delta \theta=\delta \phi=3 \mathrm{mr}$; in d), $\delta \theta=\delta \phi=4 \mathrm{mr}$; in e), $\delta \theta=\delta \phi=5$ mr. In $\mathrm{f}$ ), the same quantity for $\delta \theta=\delta \phi=5 \mathrm{mr}$, when the correlations are accounted for. The adjustable cuts used are the same in each case. 


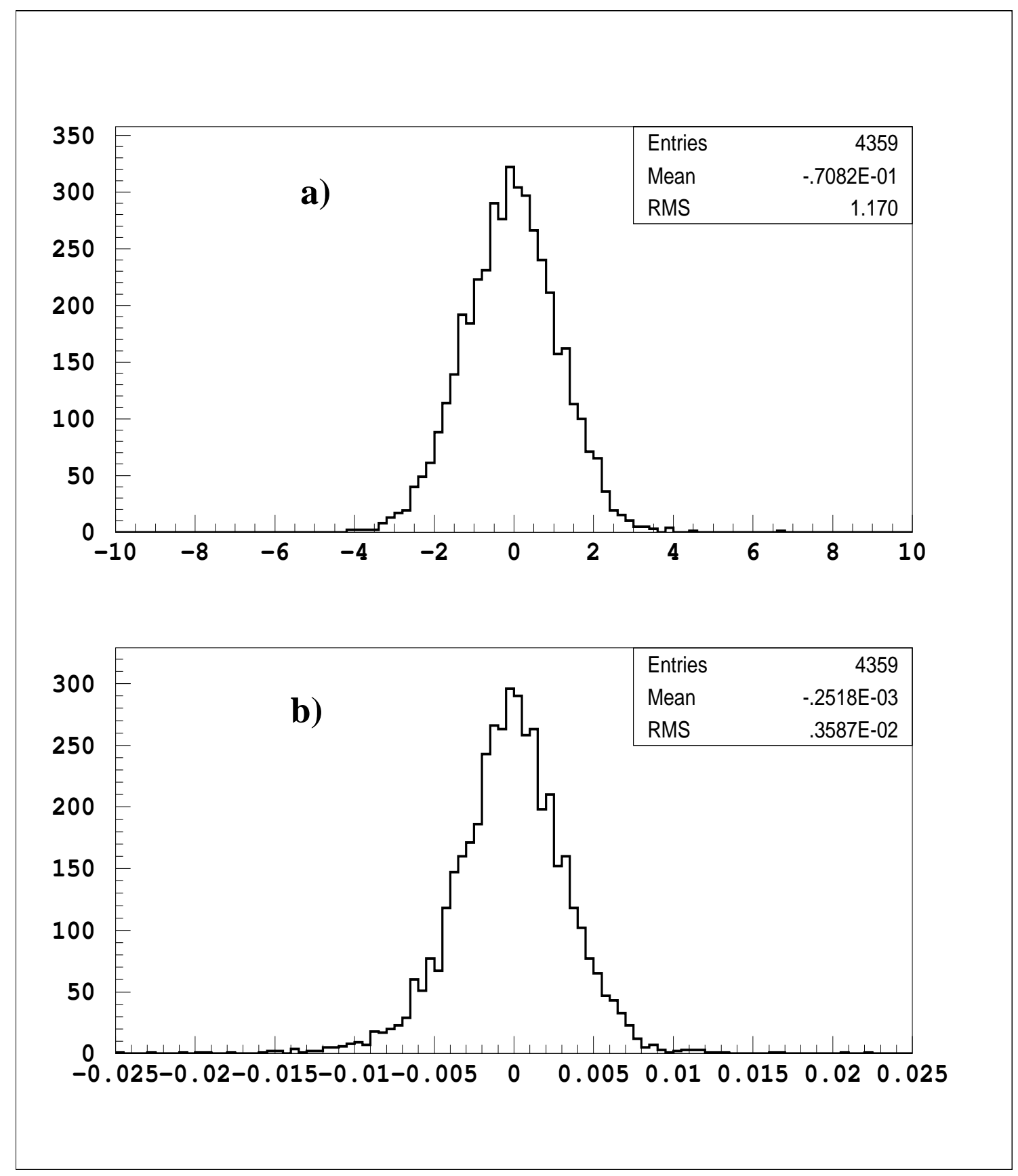

Figure 15: Reconstructed Cerenkov angle pulls (in a) and biases (in b) for the mixed particle sample, one identified additional track has been superimposed as background. 


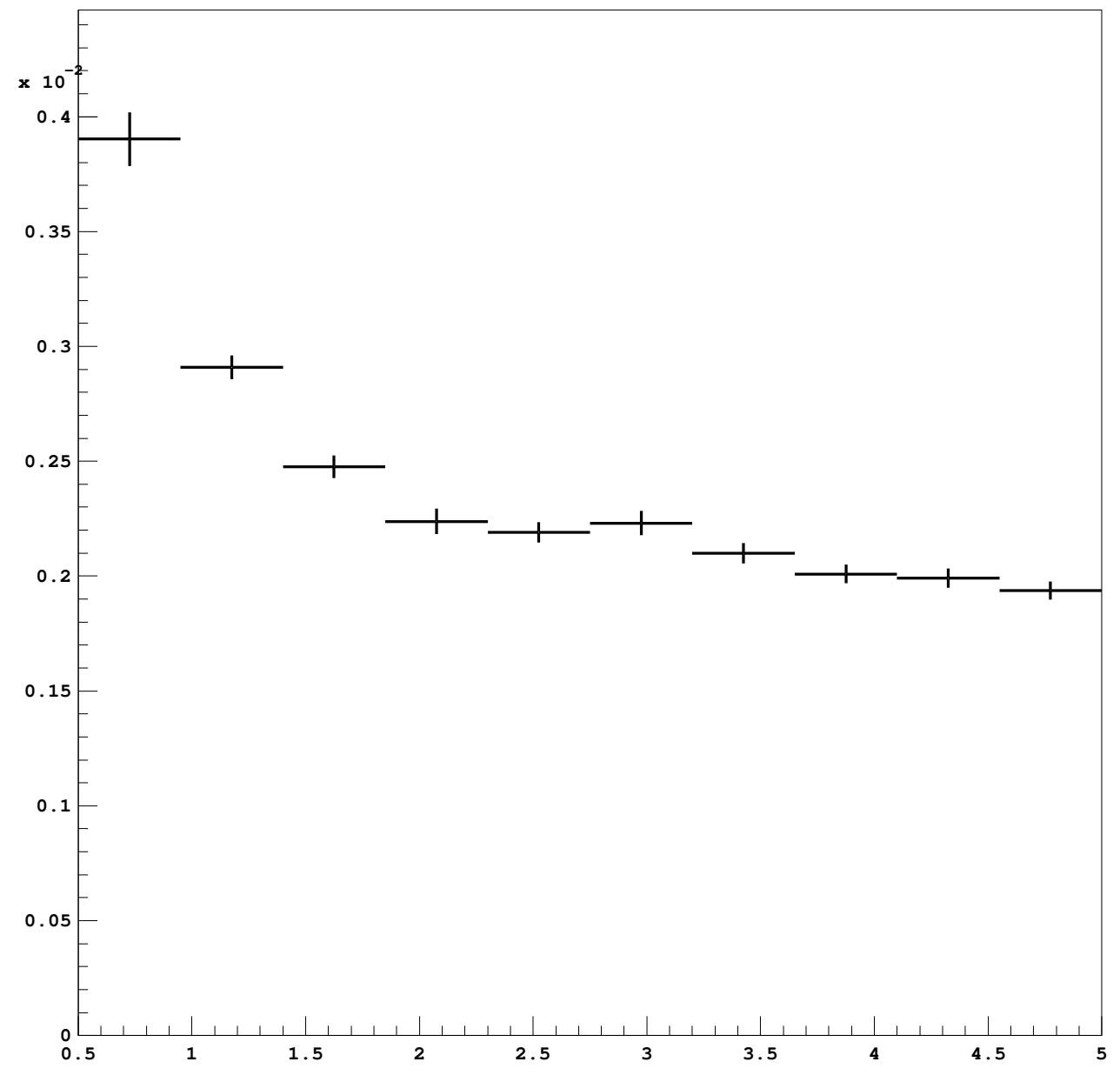

Figure 16: Theoretical Cerenkov angle mean errors (in radian units) vs track momentum $(\mathrm{GeV})$; notice the rise at low momentum, mainly due to the increase of multiple scattering effects. 

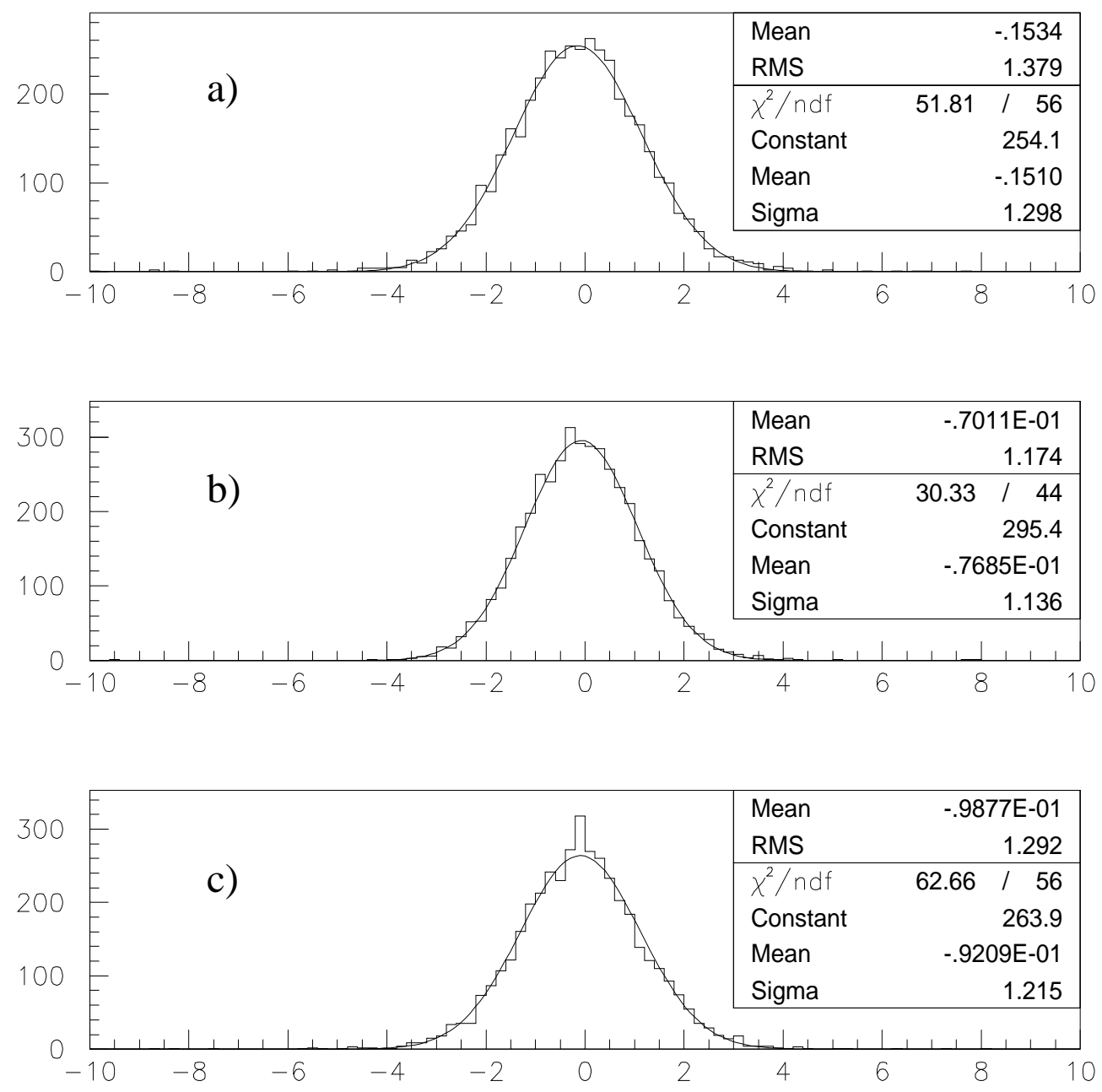

Figure 17: Effect of different types of background on the pull distributions of the reconstructed Cerenkov angle. In a), a flat random noise is superimposed to the signal track; in b), a second track is mixed with the signal track ; in c) two additional tracks are superimposed to the signal track. The effect of noise seems more important in case a). 

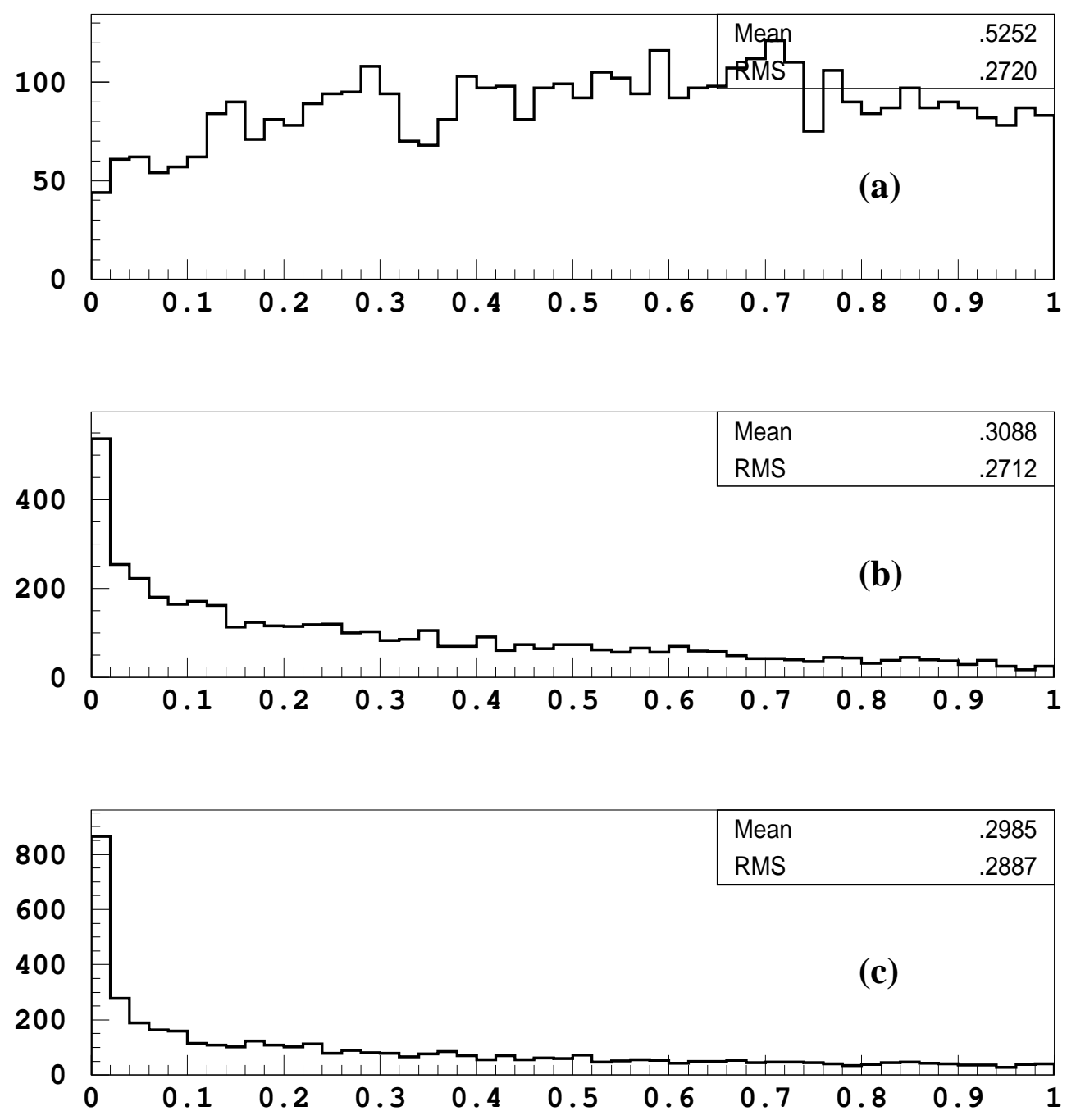

Figure 18: $\chi^{2}$ probability distribution for the sample of mixed particles when using the same set of cuts ; in (a), only signal photons are considered ; in (b), a flat random photon noise has been superimposed to signal photons; in (c), photons from one additional track (considered as background) are superimposed to the original track photons. 


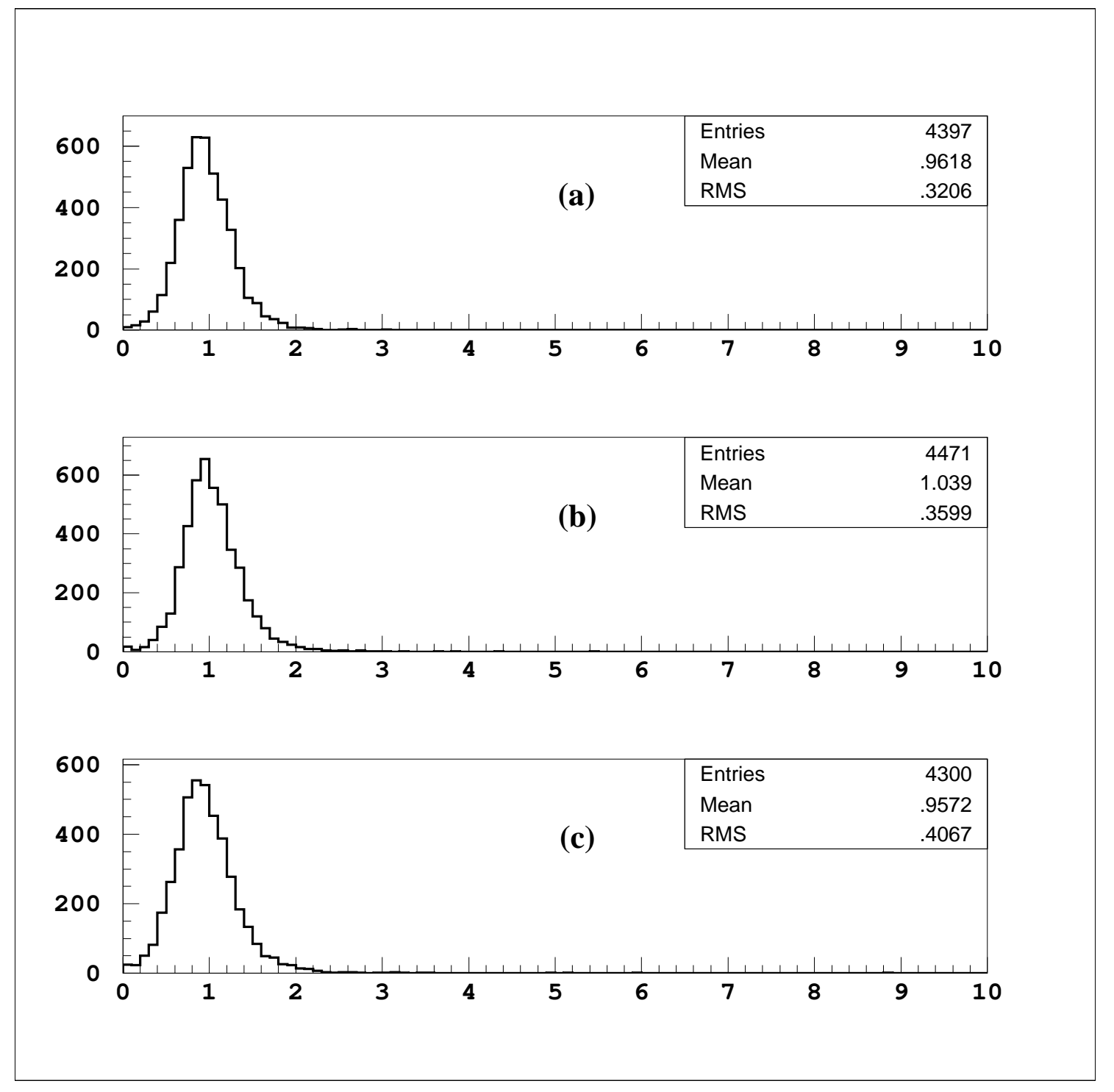

Figure 19: Adaptation of cuts to background conditions, reflected in the $\chi^{2}$ per $n_{\text {dof }}$ distribution for the sample of mixed particles when cuts have been correctly set ; in (a), only signal photons are considered ; in (b), a flat random photon noise has been superimposed to signal photons ; in (c), photons from one additional track (which existence is supposed to be known) are superimposed to the original track photons. Accordingly, the probability distributions for each of these cases are very close to be flat. 


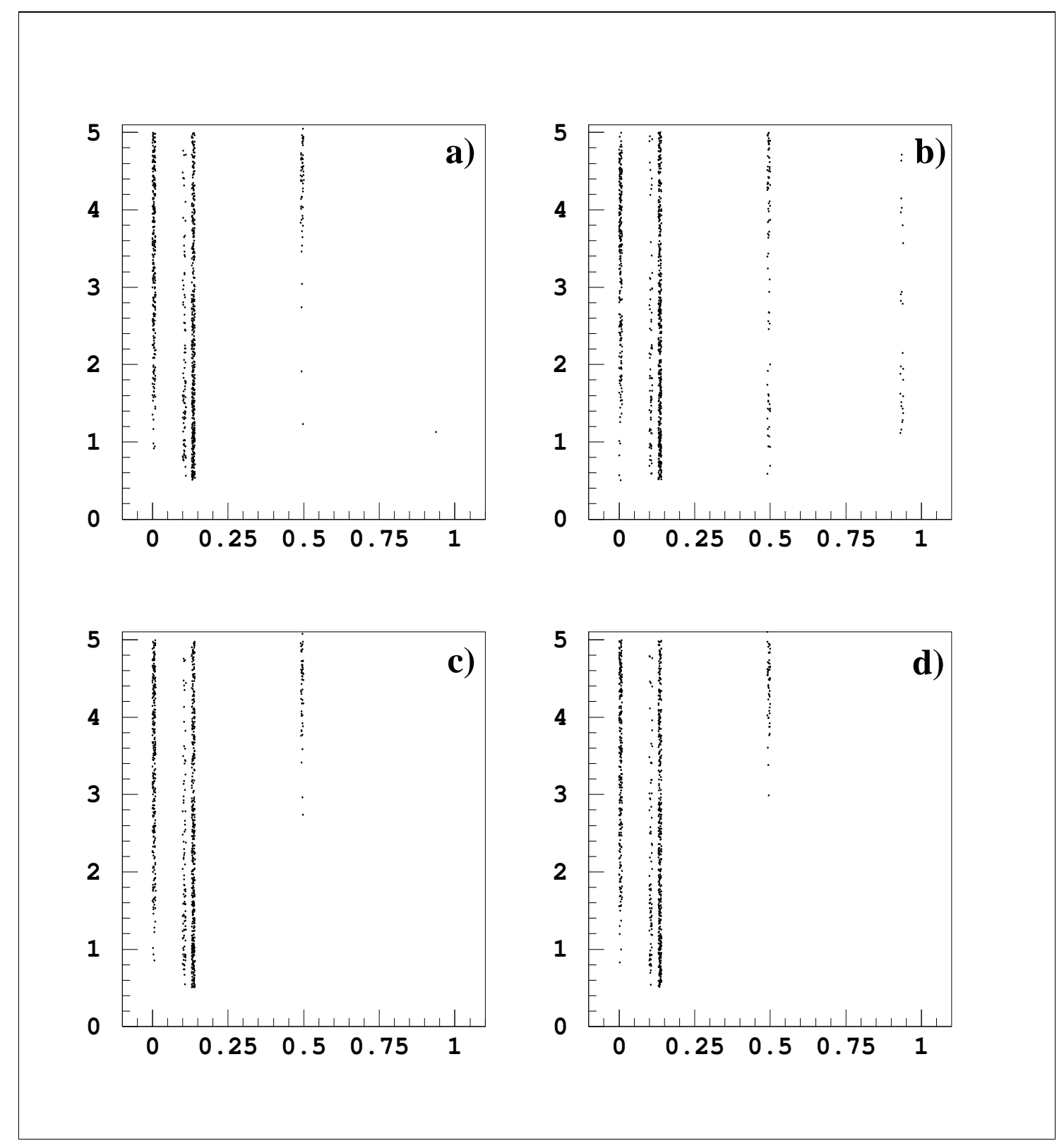

Figure 20: Effect of the $|\delta \theta|$ secondary cut and $\chi^{2}$ probability cut. The track momentum is plotted versus the identified mass ; units are $\mathrm{GeV} / \mathrm{c}$ and $\mathrm{GeV}$. In (a), the scatter plot is made for generated pion tracks with one additional identified background track; in (b), same plot when the additional track is considered as unidentified (i.e: no $|\delta \theta|$ secondary cut). In (c), $|\delta \theta|$ secondary cut applied (as in (a)), but requiring additionally a $\chi^{2}$ probability greater than 0.01 . In (d), same plot as in (c), but with a $\chi^{2}$ probability cut level of 0.03 . 


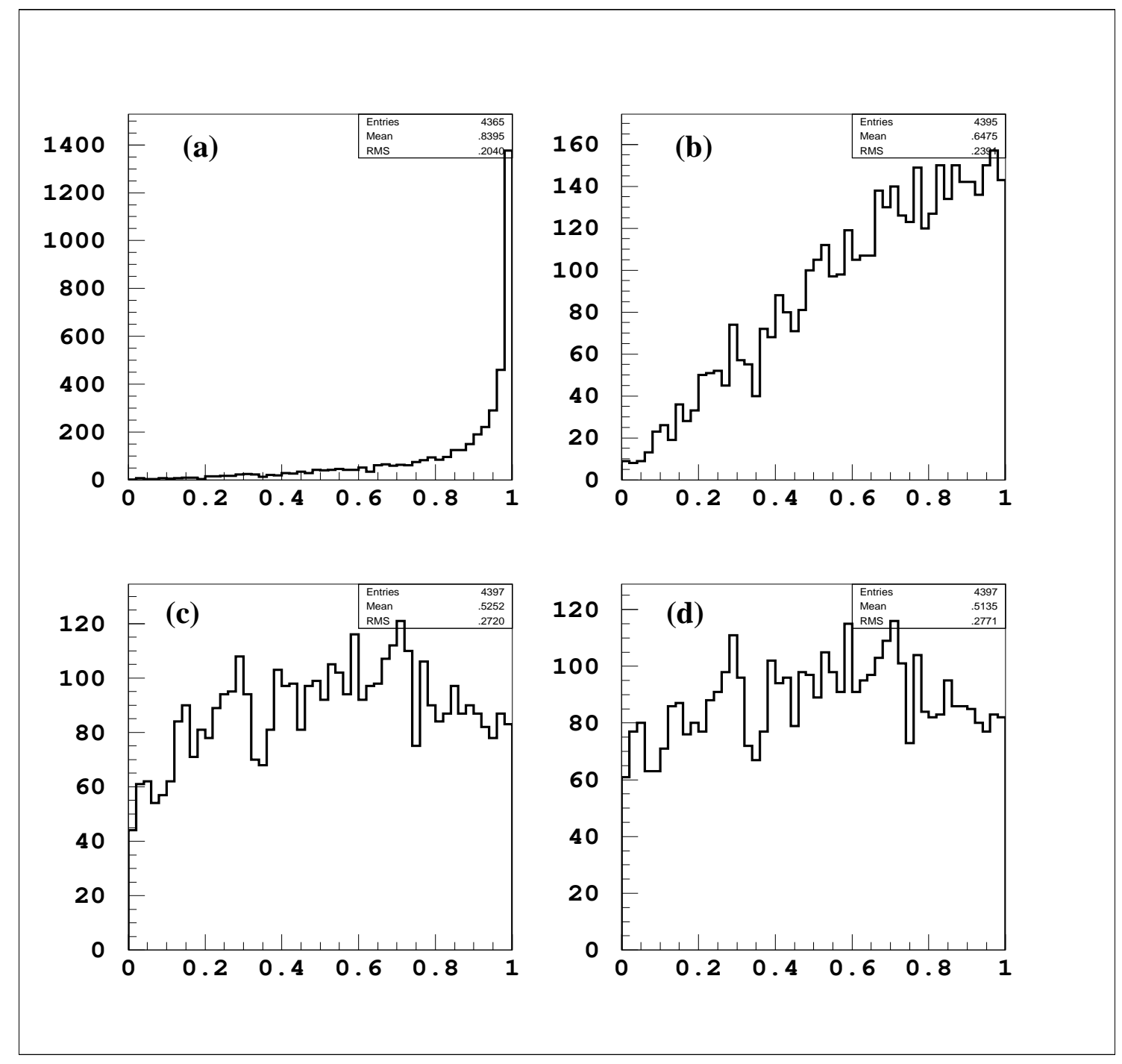

Figure 21: Effect of median cut on the $\chi^{2}$ probability distribution for the sample of mixed particles with no background of any type ; in (a), $1 \sigma$ cut ; in (b), $2 \sigma$; in (c), $3 \sigma$; in (d), $4 \sigma$. 


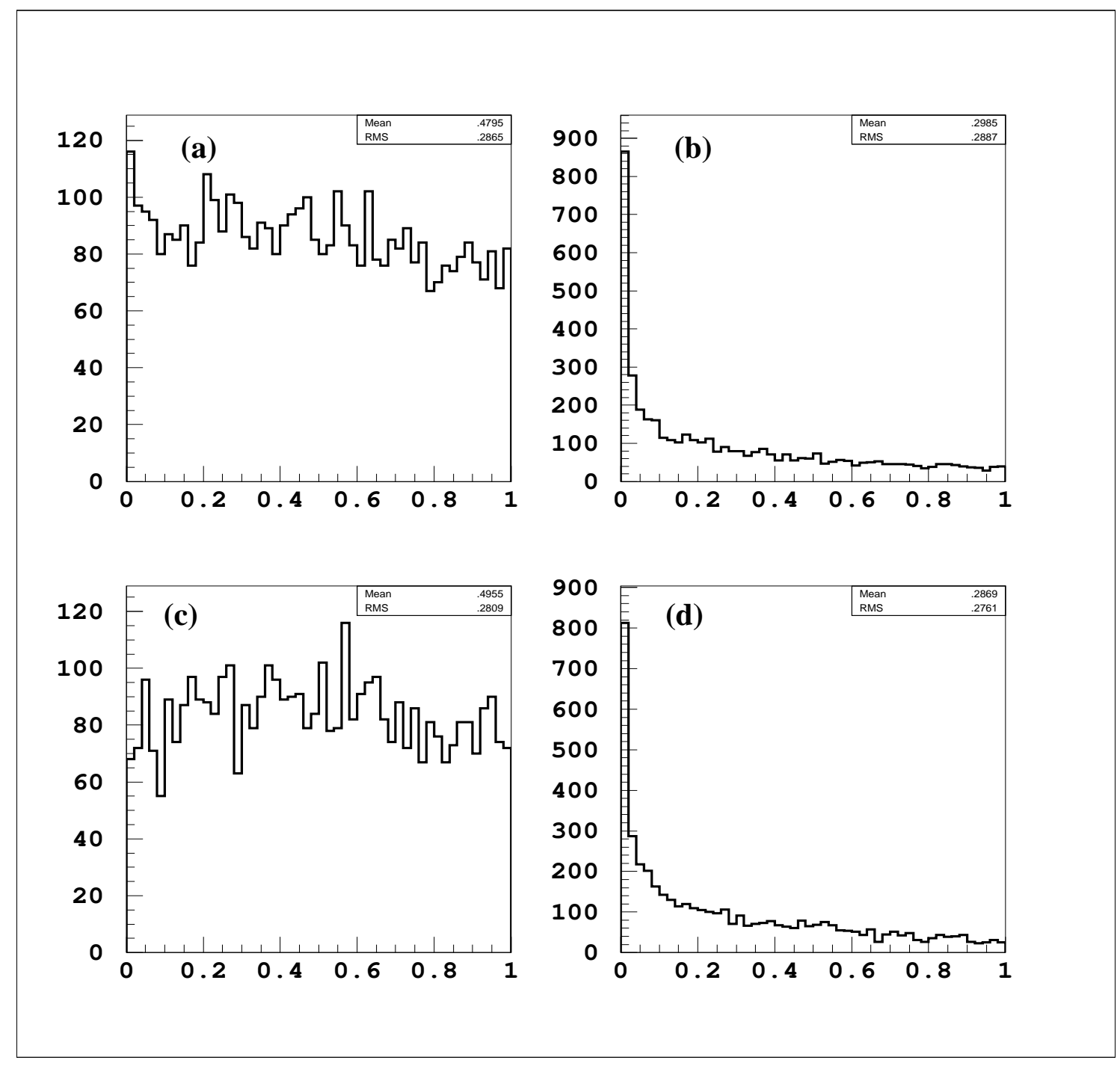

Figure 22: Effect of the $|\delta \theta|$ secondary cut. The $\chi^{2}$ probability distribution for the sample of mixed particles is shown with : (a), 1 track background and $|\delta \theta|$ secondary cut ; in (b), 1 track background and no $|\delta \theta|$ secondary cut; in (c), 2 tracks background and $|\delta \theta|$ secondary cut ; in (d), 2 tracks background and no $|\delta \theta|$ secondary cut. Note that the other cuts levels are different in cases a) and c). 


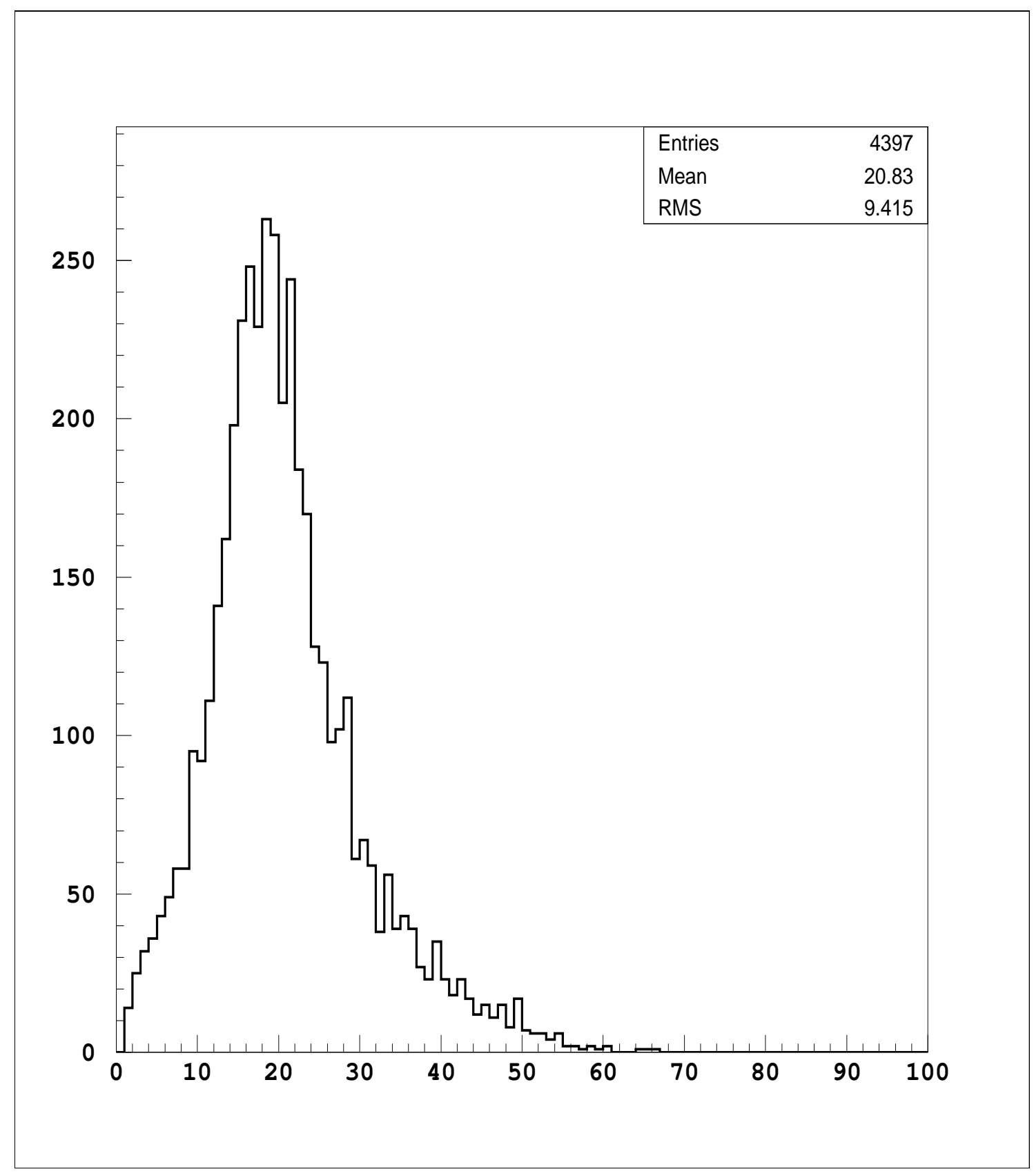

Figure 23: The number of unambiguous photons per track obtained and the end of the algorithm for the mixed particle sample (no background, no additional tracks). 